\title{
Effects of antibiotics, short-chain fatty acids and amino acids on Apoliprotein A-I transcription and synthesis in normal and inflamed HepG2 and Caco-2 cells
}

Citation for published version (APA):

Tayyeb, J. Z. (2021). Effects of antibiotics, short-chain fatty acids and amino acids on Apoliprotein A-I transcription and synthesis in normal and inflamed HepG2 and Caco-2 cells. [Doctoral Thesis, Maastricht University]. Maastricht University. https://doi.org/10.26481/dis.20210601tj

Document status and date:

Published: 01/01/2021

DOI:

10.26481/dis.20210601tj

Document Version:

Publisher's PDF, also known as Version of record

\section{Please check the document version of this publication:}

- A submitted manuscript is the version of the article upon submission and before peer-review. There can be important differences between the submitted version and the official published version of record. People interested in the research are advised to contact the author for the final version of the publication, or visit the DOI to the publisher's website.

- The final author version and the galley proof are versions of the publication after peer review.

- The final published version features the final layout of the paper including the volume, issue and page numbers.

Link to publication

\footnotetext{
General rights rights.

- You may freely distribute the URL identifying the publication in the public portal. please follow below link for the End User Agreement:

www.umlib.nl/taverne-license

Take down policy

If you believe that this document breaches copyright please contact us at:

repository@maastrichtuniversity.nl

providing details and we will investigate your claim.
}

Copyright and moral rights for the publications made accessible in the public portal are retained by the authors and/or other copyright owners and it is a condition of accessing publications that users recognise and abide by the legal requirements associated with these

- Users may download and print one copy of any publication from the public portal for the purpose of private study or research.

- You may not further distribute the material or use it for any profit-making activity or commercial gain

If the publication is distributed under the terms of Article 25fa of the Dutch Copyright Act, indicated by the "Taverne" license above, 
Effects of antibiotics, short-chain fatty acids and amino acids on Apolipoprotein A-I transcription and synthesis in normal and inflamed HepG2 and Caco-2 cells 

Effects of antibiotics, short-chain fatty acids and amino acids on Apolipoprotein A-I transcription and synthesis in normal and inflamed HepG2 and Caco- 2 cells

Jehad Tayyeb 
This thesis was possible through a Scholarship granted to Jehad Zuhair Tayyeb by University of Jeddah; a governmental university located in Jeddah.
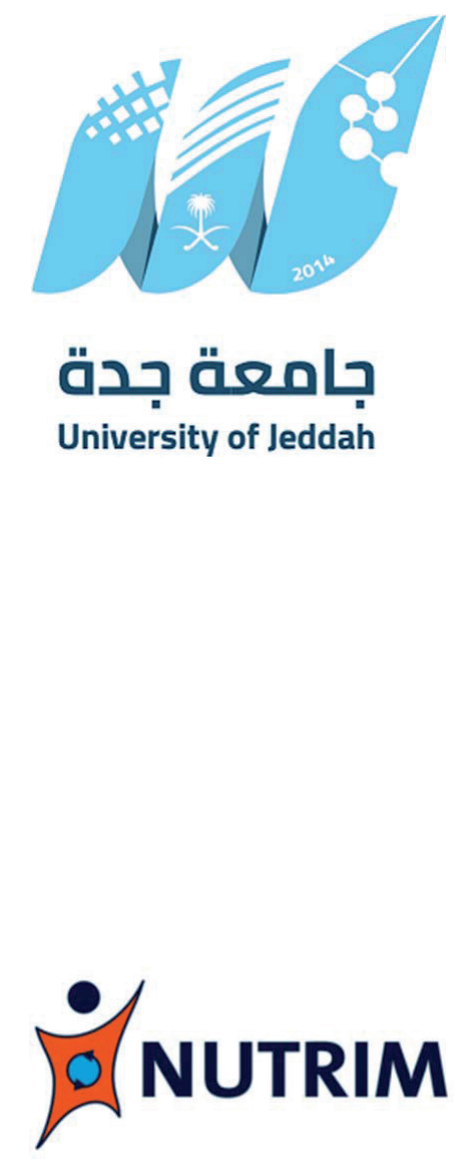

The research was performed within NUTRIM School of Nutrition and Translational Research in Metabolism. All rights are reserved. For articles published, the copyright has been transferred to the respective publisher. No parts of this thesis may be reproduced, stored in a retrieval system or transmitted in any form or by any means, without prior permission from the author.

Cover

Layout

Printed by

ISBN
ProefschriftMaken, De Bilt

Renate Siebes | Proefschrift.nu

ProefschriftMaken, De Bilt

978-94-6423-224-0

(C) Copyright Jehad Tayyeb, Maastricht 2021 


\title{
Effects of antibiotics, short-chain fatty acids and amino acids on Apolipoprotein A-I transcription and synthesis in normal and inflamed HepG 2 and Caco- 2 cells
}

\author{
DISSERTATION
}

To obtain the degree of Doctor at the Maastricht University,

on the authority of the Rector Magnificus,

Prof. Dr. Rianne M. Letschert

in accordance with the decision of the Board of Deans,

to be defended in public on Tuesday $1^{\text {st }}$ of June 2021, at 10.00 hours

by

Jehad Zuhair Tayyeb

born on December $3^{\text {rd }} 1985$, United Kingdom 


\section{Promotor}

Prof. Dr. J. Plat

Prof. Dr. Ir. R.P. Mensink

\section{Co-promotor}

Dr. H.E. Popeijus

\section{Assessment Committee}

Prof. Dr. E.C.M. Mariman (Chair)

Prof. Dr. R. Shiri-Sverdlov

Dr. E. Canfora

Prof. Dr. S.A.H. Kersten (Wageningen University)

Prof. Dr. J. Garssen (Utrecht University) 


\section{Table of contents}

Chapter 1 General introduction 7

Chapter 2 The effects of short-chain fatty acids on the transcription and 27 secretion of apolipoprotein A-I in human hepatocytes in vitro

Chapter 3 Amoxicillin modulates ApoA-I transcription and secretion, $\quad 43$ predominantly via PPAR $\alpha$ transactivation inhibition

Chapter 4 Short-chain fatty acids (except Hexanoic acid) lower NF-kB transactivation, which rescues inflammation-induced decreased Apolipoprotein A-I transcription in HepG2 cells

Chapter 5 Butyric acid added apically to intestinal Caco-2 cells elevates 81 hepatic ApoA-I transcription and rescues lower ApoA-I expression in inflamed HepG2 cells co-cultured in the basolateral compartment

Chapter 6 Differential effects of individual amino acids on PPAR $\alpha$ transactivation, ApoA-I transcription and (pro)-ApoA-I secretion

Chapter 7 General discussion

Chapter 8 Summary

Chapter 9 Impact paragraph

Abbreviations 153

Acknowledgements 157

Curriculum vitae 165

List of publications 166 

General introduction 



\section{Atherosclerosis and cardiovascular disease}

Cardiovascular diseases (CVD) are still the leading cause of morbidity and mortality worldwide (1). The prevalence of CVD is estimated to even further increase by $18 \%$ in 2030 (2). Therefore, screening for existing CVD risk via established and novel biomarkers as well as developing new intervention strategies to prevent and treat CVD received a lot of the attention over the past decades. The most important strategy to reduce the occurrence of CVD is to prevent atherosclerotic lesion development. Known risk factors for atherosclerotic lesion development are dyslipidemia, hypertension, diabetes, elevated BMI, a lack of physical inactivity and genetic factors $(3,4)$. Dyslipidemia is a condition of a disturbed and unbalanced lipoprotein metabolism as shown by elevated low-density lipoprotein (LDL) cholesterol and triglyceride (TG) concentrations or reduced highdensity lipoprotein (HDL) cholesterol concentrations (5). Moreover, these metabolic abnormalities contribute to the accumulation of (oxidized) cholesterol depositions within the artery wall, resulting in increased atherosclerotic lesion development (6). Moreover, atherosclerosis is not only characterized by the progressive accumulation of cholesterol but also of macrophages within the inner lining of blood vessels $(7,8)$, which relates to the fact that it is also a chronic inflammatory condition (9) in which many additional factors and immune cells play a role. Altogether these processes lead to a condition called endothelial dysfunction (Figure 1.1), which - when untreated - progresses further towards the formation of an atherosclerotic plaque.

\section{The role of $\mathrm{LDL}$ and $\mathrm{HDL}$ in atherosclerosis}

It is well-known that atherosclerotic plaque formation correlates with serum cholesterol concentrations. Individuals with high LDL cholesterol concentrations or low HDL cholesterol levels are considered at high risk of atherosclerosis $(12,13)$. There is an overwhelming amount of data showing that serum LDL cholesterol concentrations are strong and positively linked to CVD risk, and several reviews demonstrated the causal link between LDL-C and CVD $(14,15)$. Moreover, it was also shown that any decrease in LDL-C, independent of the size of the reduction, is associated with a decrease in CVD risk. An important message came from a meta-analysis including several drug and dietary intervention trials in which it was demonstrated that the benefit of lowering LDL-C is similar for all strategies, either via pharmacological or lifestyle approaches (16). Recent studies have shown that lowering LDL cholesterol concentrations alone is often not enough to fully prevent or stop atherosclerosis development. Therefore, an increased interest emerged to find additional therapies via targeting other lipids and lipoproteins that 


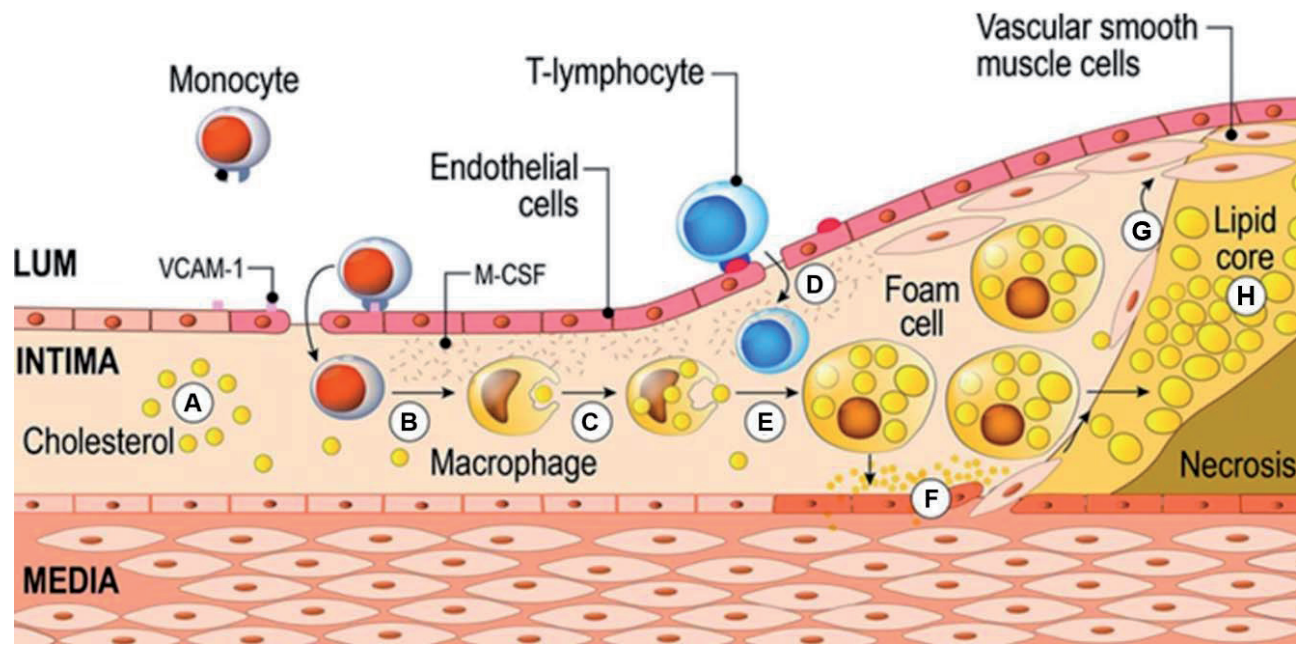

Figure 1.1. The process of atherosclerosis. When LDL becomes trapped in the sub-endothelial space (A), it may undergo oxidative modification, forming oxy-LDL. Consequently, the endothelial cells express various leukocyte adhesion molecules such as VCAM1 and stimulate mononuclear cells such as monocytes and $\mathrm{T}$-cells to attach to the endothelium and enter subsequently the intima, where they differentiate $(\mathrm{B}, \mathrm{C})$ into macrophages $(9,10)$. T-lymphocytes join macrophages in the intima (D), and form lipid laden foam cells (E). The inflammatory condition stimulates the development of vascular smooth muscle cells, forming an atherosclerotic plaque (F, G). Eventually, the accumulation of cells debris, cytokines, chemokines, and apoptotic bodies results in necrotic core formation (H). Adapted from Minelli et al. (11).

also associated with CVD risk, such as HDL cholesterol (17) or triglycerides (13). HDL cholesterol concentrations are inversely correlated with CVD risk. Previous cross-sectional studies have reported that a $1 \mathrm{mg} / \mathrm{dl}(0.03 \mathrm{mM} / \mathrm{L})$ higher HDL cholesterol concentration associates with a 3\% lower CVD risk (18). Moreover, recent studies have also shown reductions in CVD risk by using HDL cholesterol elevating interventions, however this effect is not consistent (17). HDL particles are the smallest and densest lipoproteins, which differ in size, shape and lipid composition. Proteins form approximately 50\% of an HDL particle, and $65-70 \%$ of this protein fraction is Apolipoprotein A-I (ApoA-I). In other words, ApoA-I is the main component of HDL (19). HDL has different functions: it can act as an antioxidant, anti-inflammatory, and antithrombotic agent. On the other hand, HDL particles can also become pro-inflammatory and pro-atherogenic, mainly when an acute phase or a chronic phase of the systemic inflammatory state is active (20). The main atheroprotective function of HDL is to enhance the removal of cholesterol from macrophages within the artery wall and to transport this cholesterol to the liver for excretion (21). This process is called reverse cholesterol transport (RCT) (Figure 1.2). Therefore, a promising strategy to reduce the unfavorable atherosclerosis development is to increase the RCT and/ or cholesterol efflux capacity. 




Figure 1.2. Schematic overview of HDL metabolism and the reverse cholesterol transport (RCT) pathway. First, lipid free ApoA-I is produced in the liver and small intestine, followed by obtaining cholesterol and phospholipids via ATP Binding Cassette Subfamily A Member 1 (ABCA1) mediated efflux from the liver to form nascent pre- $\beta$ HDL particles (22). Next, pre- $\beta$ HDL particles acquire additional free cholesterol and phospholipids from macrophages in the extrahepatic tissues via ABCA1-mediated efflux generating particles that are more cholesterol enriched (22). Then, lecithin cholesterol acyltransferase (LCAT) enzyme esterifies the free cholesterol molecules to form cholesteryl ester, which migrate to the core of the HDL particle to form mature HDL particles (23). Lastly, HDL particles bind to the SR-B1 receptor to bring the obtained cholesterol to the liver which is followed by the production of bile acids $(23,24)$. Additionally, HDL particles transfer its cholesterol content to LDL via CETP enzyme and then these lipoproteins can be bind to the liver receptors and deliver their cholesterol content (24). Obtained from Servier Medical Art (https://smart.servier.com).

\section{HDL functionality and ApoA-I}

As already mentioned in the previous section, recent studies have shown that elevating HDL cholesterol concentrations is not always beneficial $(25,26)$. Moreover, when the composition of HDL is changed, it can even exert a negative effect on the vasculature (27). Therefore, over the past decade, it became evident that HDL functionality is more important in terms 
of HDL-related athero-protective activities than the HDL cholesterol concentrations itself. The functionality of HDL is defined as its ability to show anti-atherogenic properties, such as regulating the RCT process, which is the main athero-protective function of HDL (28). Moreover, HDL cholesterol efflux capacity from macrophages shows an inverse correlation with the CVD (25). As mentioned above, ApoA-I is the main protein constituent of HDL particles and in contrast to HDL cholesterol concentrations, ApoA-I levels have so far remained a strong predictor of CVD risk, both cross-sectional (29) as well as in interventions aiming at an elevation of ApoA-I $(30,31)$. As ApoA-I is the ligand for ABCA1 mediated cholesterol efflux, ApoA-I is the main driver of RCT and therefore elevating ApoA-I concentrations is a new promising strategy to reduce atherosclerosis development. Newly synthesized ApoA-I is first produced as a Pre-pro-ApoA-I protein after the transcription of the ApoA-I gene within liver or small intestine cells. First, this Pre-pro-ApoA-I is cleaved in the endoplasmic reticulum in response to the signal peptidase process (32). Consequently, as a result of this cleavage, intracellular pro-ApoA-I is formed and secreted by the cells into the extracellular space. Next, proapoA-1 undergoes post-translational proteolytic cleavage after secretion resulting in removal of the pro-segment and conversion into mature ApoA-I, a process that is regulated by the action of bone morphogenetic protein-1 (BMP-1) and procollagen c-proteinase enhancer-2 protein (PCPE2) $(33,34)$. After that, the c-terminal domain of this lipid poor ApoA-I starts an interaction with the ABCA1 transporter within the macrophages, as such facilitating the uptake of the cholesterol which is the initial step of the RCT process $(35,36)$.

\section{Elevating of ApoA-I therapeutic effects}

The question is how strong the evidence is that elevating ApoA-I concentrations, and probably also HDL functionality, lowers CVD risk. Recent studies indeed suggest that elevating ApoA-I inhibits the progression of atherosclerosis and can protects against development of $\operatorname{CVD}(37,38)$. Human clinical studies have shown that ApoA-I infusion associated with increased RCT, reduced cardiac inflammatory conditions and enhanced phospholipid oxidation (39-41). Therefore, new drugs targeting ApoA-I were developed and investigated in several clinical studies (42). Typical examples of such drugs are shown in Table 1.1, where they are summarized based on their therapeutic approaches.

As mentioned, numerous studies have shown that ApoA-I was associated with multiple beneficial effects on CVD. For example, Nissen et al. found that ApoA-I-Milano infusion significantly regressed atherosclerosis in patients with coronary heart disease (Table 1.1. A1) (43). Other studies showed a reduction in total atheroma volume by $4.2 \%$ in patients with 
acute coronary syndrome after intravenous ApoA-I (ETC-216) treatment (44). Moreover, treatment with purified native ApoA-I (CSL-111) decreased atheroma volume by $3.4 \%$ in coronary disease patients (45). Furthermore, the use of RVX-208 increased ApoA-I and pre $\beta$-HDL concentrations in plasma by $10 \%$ and $42 \%$, respectively, and additionally enhanced the cholesterol efflux capacity (Table 1.1. A2) (46). ApoA-I mimetics are short synthetic peptides with an amphipathic helix, that imitate the biological functions and the structure of ApoA-I (47). These peptides fragments which can be infused or taken orally (42), are very promising as a potential therapies for $\operatorname{CVD}(48,49)$. In a recent study, Dunbar et al. have found that oral ApoA-I mimetic (D-4F) suppressed the HDL inflammatory index in high-risk CVD patients (Table 1.1. C1) (50). Thus, the evidence of these clinical trial clearly show that elevating ApoA-I concentrations lowers CVD risk and therefore it is evident that we need a better understanding of the process underlying ApoA-I transcription and production and identify novel compounds that can be used to elevate this process.

Table 1.1. ApoA-I different therapeutic strategies in the clinical studies (42)

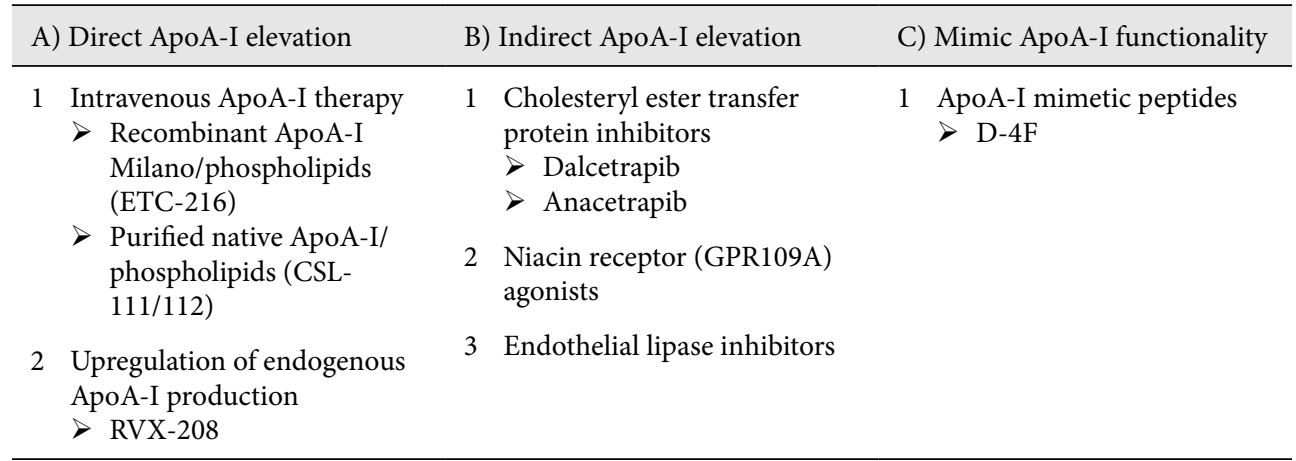

\section{ApoA-I transcription}

ApoA-I contains 243 amino acids and is mainly produced in the liver and small intestine cells. The ApoA-I gene is located at chromosome 11 and its regulation mainly occurs at the transcriptional level (51). Several transcription factors and elements regulate ApoA-I transcription by activating or inhibiting ApoA-I gene expression in response to metabolic or hormonal changes (52) (Figure 1.3). This regulatory process also requires the presence of the ApoC-III enhancer, particularly for intestinal ApoA-I transcription (53). There is ample evidence that peroxisome proliferator-activated receptor alpha (PPARa) activators such as fibrates are able to induce ApoA-I gene expression $(54,55)$. Moreover, also Bromodomain and Extra-Terminal (BET) inhibitors significantly increased ApoA-I transcription and production. For example, the BET inhibitors RVX-208 and JQ1(+) increase ApoA-I 
expression via inhibiting the binding of BET proteins to the acetylated histone residues $(56,57)$. In addition, several hormones were reported to modulate ApoA-I gene expression such as thyroid hormone, estrogen and glucocorticoid (58). Taken together, discovering natural compounds which can increase ApoA-I transcription and HDL functionality via these targets is needed.

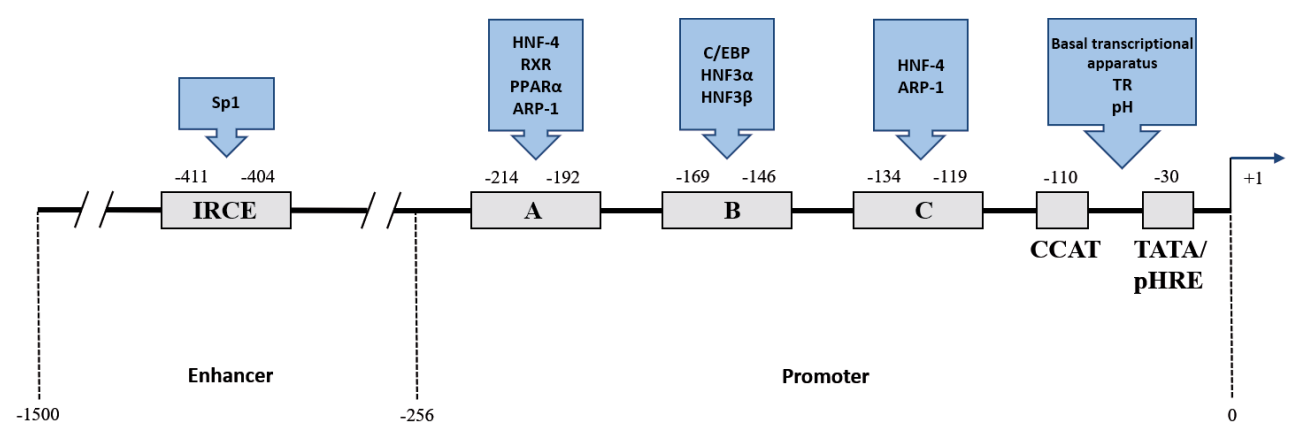

Figure 1.3. The ApoA-I promoter/enhancer model with selective activators or inhibitors. The sites A, B and $\mathrm{C}$ are essential for ApoA-I gene transcription. The PPAR response element (PPRE) is located on site A, which is a binding element for the transcription factor PPARa (59). Moreover, both sites A and C contain ARP-1 (ApoA-I repressor protein-1) and hormone response elements (HRE), which are binding elements of hepatic nuclear factor 4 (HNF-4) (60). Although both A and $\mathrm{C}$ sites contain HRE binding elements, the retinoid $\mathrm{X}$ receptor (RXR)/PPARa heterodimer binds only to site A (61). Furthermore, CCAAT-enhancer binding proteins (C/EBP), HNF3 $\alpha$ and $\mathrm{HNF} 3 \beta$ bind to site $\mathrm{B}(62,63)$. In addition, an insulin response core element (IRCE) is located on the enhancer site, which binds to the ubiquitous transcription factor Sp1, resulting on ApoA-I gene production by insulin (64).

\section{Link between inflammation and ApoA-I concentrations}

Inflammation leads to several structural and metabolic alterations in lipid and lipoproteins, which are associated with increased CVD risk (65). For example, inflammation results in a reduction of HDL cholesterol and HDL particle concentrations and most important even in the formation of dysfunctional HDL particles, which altogether eventually leads to inflammation induced RCT impairment (66). The innate immune system is known as the first line of defense against infections, and the main action of it is to fight against host of organisms by the activation of the oxidative state (67). This action is known as acute phase response, which occurs, amongst other tissues, in the liver (67). A number of cytokines such as Interleukin-8 (IL-8) stimulate the acute phase response in the liver (68). These inflammatory cytokines suppress the hepatic expression of ApoA-I and enhance the production of serum amyloid A, which becomes the major protein component of HDL (69-71). Therefore, the question is how to elevate ApoA-I concentrations during these inflammatory conditions. 


\section{SCFAs (physiology, production, absorption and function)}

It is well-known that the composition of the intestinal microbiota plays a major role in the colonic health and contributes to the development of various conditions and diseases such as type 2 diabetes (72) and the metabolic syndrome (73). Besides a direct role on (intestinal) physiology for example via regulating the (local) host immune response (74), these microorganisms have enzyme activity which can break down carbohydrates and fibers and consequently catalyze the formation of different useful metabolites (74). For example, after intake of a meal that contains soluble fibers, the anaerobic intestinal microbiota produces short-chain fatty acids (SCFAs) by fermentation of these fibers (75). Notably, the fermentation of oat bran and resistant starch is assumed to result in the highest amounts of SCFAs (76). SCFAs are fatty acids that consist of carbon atoms with a length ranging from 2 to 6 , i.e. acetic acid (C2), propionic acid (C3), butyric acid (C4), valeric acid (C5) and hexanoic acid (C6) $(77,78)$. The cecum is the major site of SCFAs production, in addition to the ascending, transverse, descending and sigmoid colon $(76,79)$. However, although SCFAs concentrations are considerably higher (80) (-131 mmol/ $\mathrm{kg}$ of intestinal content) in the colon, there are certainly also SCFAs formed and present within the terminal ileum, though in lower concentrations $(13 \mathrm{mmol} / \mathrm{kg}$ of intestinal content) (80). The absorption of SCFAs from the intestinal lumen is effective and fast with only $5 \%$ of SCFAs being excreted into the feces (79). The colonic absorption occurs via the apical membrane of the epithelial cells through several transporters, including the sodium-coupled monocarboxylate transporter 1 and hydrogen-coupled monocarboxylate transporters (MCT) $(81,82)$. Although most SCFAs are absorbed by the colonocytes and locally used as fuel, part of the SCFAs are released from the gut and transferred via the mesenteric veins to the portal vein and the liver (83). Eventually, the remaining SCFAs, which are not retained in the liver, are transported into the circulation (84). Human studies in which portal SCFAs concentrations were measured are scarce and only carried out in patients during surgery. For example, Bloemen et al. found that propionate and butyrate concentrations in the portal plasma were 30.3 and $30.1 \mu \mathrm{mol} / \mathrm{l}$, respectively (83). Therefore, it is unknown whether these concentrations also reflect the healthy situation. In terms of underlying mechanisms, recent studies have revealed that SCFAs might affect enterocytes by binding to $G$ protein coupled receptors (GPCRs) and activate intracellular signaling. For SCFAs, these GPCRs are FFAR2 (GPR43) and FFAR3 (GPCR41) (84). Interestingly, both receptors are also expressed in human liver, adipose tissue and skeletal muscles, which suggests that SCFAs could also influence (metabolic) processes in these peripheral tissues (85). The physiological functions of SCFAs include energy production for the colonocytes and the reduction of the gut luminal $\mathrm{pH}$, which increases mineral absorption and inhibits 
pathogens growth (86). In addition, SCFAs prompt expression of many genes and regulate the secretion of hormones and GI peptides such as glucagon-like peptide 1 (GLP-1) (87). Moreover, SCFAs might also lower cholesterol synthesis and modulate glucose metabolism (88) .

\section{SCFAs role in metabolic and cardiovascular health}

Many epidemiological studies have suggested that SCFAs could play a role in the prevention and treatment of metabolic diseases $(76,89-92)$. Systemic SCFAs are thought to contribute to a wide range of health benefits including improvements in glucose homeostasis, blood lipid profiles and reduction of body weight $(77,78)$. In addition, SCFAs exert anti-inflammatory effects, which might contribute to explaining the reduced development of atherosclerosis $(93,94)$. This is in line with earlier observations suggesting a protective role for SCFAs in CVD development and other inflammatory diseases (79). The anti-atherosclerotic effects of SCFAs are assumed to be mediated by modulating pro-inflammatory cytokine production, endothelial dysfunction and oxidative stress (95). In animal studies, Bartolomaeus and coworkers have recently shown that propionate supplementation reduced atherosclerosis (96). Moreover, the supplementation of propionate and butyrate in diabetic animals reduced hepatic lipid accumulation and improved glucose tolerance (97). A recent human study reported that dietary $\mathrm{C} 4$ supplementation was associated with improved blood pressure through a reduction in inflammation (98). Furthermore, colonic infusion of SCFAs increased energy expenditure and fat oxidation, while decreased adipose tissue lipolysis in overweight subjects (91), and propionate has been shown to modulate liver lipid content in NAFLD patients (99). Altogether, the findings so far are suggestive that SCFAs can influence metabolic processes that prevent the development of CVD, but intervention studies in humans are needed to come to more firm conclusions.

\section{The possible relationship between SCFAs and ApoA-I}

Clinical studies revealed that antibiotics disturbed gut microbiota and altered lipid and lipoprotein metabolism (100-102). In a recent human trial carried out in our group, we found that amoxicillin treatment reduced HDL cholesterol concentrations (101). Since ApoA-I is the major structural protein form HDL particles, the question is whether antibiotics could also modulate ApoA-I transcription and synthesis. Theoretically, the effects of antibiotics on ApoA-I expression and secretion can be the result from direct effects on ApoA-I gene transcription or indirect effects through affecting microbiota composition and subsequently 
intestinal SCFAs production and SCFAs concentrations that reach the liver. For example, the intake of vancomycin resulted in a reduction of SCFAs concentrations in human's plasma (100). An additional indication supporting this assumption comes from a recent study in which it was shown that SCFA supplementation increased HDL cholesterol concentrations in hamsters (103). Another indication for a potential relationship between SCFAs and ApoA-I is based on the fact that fatty acids can bind strongly to PPARa, a transcription factor that plays an essential role in the regulation of ApoA-I expression $(104,105)$. Based on our previous data regarding the effects of long chain fatty acids on PPAR activation and ApoA-I transcription, it is likely to suggest that SCFAs also can bind to PPARa, which eventually led to PPAR $\alpha$ transactivation and ApoA-I transcription (104).

\section{Exploring effects of natural compounds (i.e. amino acids) on ApoA-I}

As mentioned, increasing ApoA-I production is a promising strategy to reduce the CVD risk $(37,38)$ and therefore, studies exploring the potential effects of dietary compounds that can elevate ApoA-I transcription is needed. Thus, we also focused on natural compounds with the ability to stimulate PPARa, a transcription factor, that regulates ApoA-I expression $(104,105)$. Amino acids are interesting candidates since there are indications that amino acids can modulate PPAR $\alpha$ expression (106). For example, leucine was found to significantly increase PPARa expression in skeletal muscle myotubes (106). Moreover, leucine also significantly reduced p-P65-NFkB expression leading to reduced IL6 mRNA expression (106). Furthermore, other studies showed that leucine, glutamine and proline all reduced IL-8 production, probably via NF- $\kappa$ B inhibition, in HepG2 cells (107), a process in which PPARa is also involved (108). Therefore, amino acids could be additional dietary compounds that play a role in regulating ApoA-I expression and there is a clear need to further explore this hypothesis.

\section{Outline of this dissertation}

The aim of this dissertation was to study the effects of a number of variables on ApoA-I production in vitro. For example, we observed in an earlier placebo controlled human intervention study carried out in our group that antibiotics changed serum HDL cholesterol concentrations. Since do novo ApoA-I production is a possible driver behind elevations in serum HDL cholesterol, we speculated that this observation could be a direct effect the antibiotic or otherwise an indirect effect related to changes in SCFA's due to antibiotic induced changes in microbiota composition. Therefore, in chapter 2 we examined the dose- 
response relationship between several SCFAs and ApoA-I transcription and secretion in HepG2 cells. In addition, we evaluated the molecular mechanisms underlying theses effects on ApoA-I such as PPARa transactivation and BET inhibition. In chapter 3, we investigated the direct effects of different classes of antibiotics on ApoA-I transcription and secretion in hepatocytes and enterocytes. Moreover, we addressed the potential involvement of BET inhibition, PPARa transactivation and ER stress on these antibiotic-induced changes in ApoA-I transcription. In chapter 4 we focused on the potentially protective effects of SCFAs on inflammatory pathways (NF- $\kappa$ B transactivation and IL-8 secretion) in HepG2 cells exposed to a cytokine cocktail, which is known to lower ApoA-I expression. We also again evaluated the possible mechanisms underlying these anti-inflammatory effects of SCFAs on ApoA-I transcription. In chapter 5, we aimed to study the possible existence of a cross-talk between enterocytes and hepatocytes on ApoA-I transcription. Therefore, we compared the effects of C4 on ApoA-I transcription in Caco-2 and HepG2 cells when cultured alone to the effects when both cells were co-cultured together in a transwell system. Furthermore, till now we fully focused on the effects of dietary lipids on ApoA-I transcription. However, we broadened our horizon and explored in chapter $\mathbf{6}$ the potential effects of different amino acids on ApoA-I transcription and secretion in HepG2 cells. We again also investigated the underlying pathways which might induce these effects of amino acids on ApoA-I production. Finally, in chapter 7, the main outcomes of all these studies are discussed and placed into broader perspective.

\section{References}

1. Olson, M.; Chambers, M.; Shaibi, G. Pediatric markers of adult cardiovascular disease. Curr. Pediatr. Rev. 2017, 13, 255-259.

2. Valanti, E.-K.; Dalakoura-Karagkouni, K.; Sanoudou, D. Current and emerging reconstituted HDL-apoA-I and HDL-apoE approaches to treat atherosclerosis. J. Pers. Med. 2018, 8, 34.

3. Dahlöf, B. Cardiovascular disease risk factors: epidemiology and risk assessment. Am. J. Card. 2010, 105, 3A-9A.

4. Badimon, L.; Vilahur, G. LDL-cholesterol versus HDL-cholesterol in the atherosclerotic plaque: inflammatory resolution versus thrombotic chaos. Ann. N. Y. Acad. Sci. 2012, 1254, 18-32.

5. Ahmed, S. M.; Clasen, M. E.; Donnelly, J. E. Management of dyslipidemia in adults. Am. Fam. Physician 1998, 57, 2192-2204, 2207-2198.

6. Miller, M. Dyslipidemia and cardiovascular risk: the importance of early prevention. QJM-Int. J. Med. 2009, 102, 657-667.

7. Torres, N.; Guevara-Cruz, M.; Velázquez-Villegas, L. A.; Tovar, A. R. Nutrition and atherosclerosis. Arch. Med. Res. 2015, 46, 408-426.

8. Ma, J.; Li, H. The role of gut microbiota in atherosclerosis and hypertension. Front. Pharmacol. 2018, 9, 1082.

9. Frostegård, J. Immunity, atherosclerosis and cardiovascular disease. BMC Med. 2013, 11, 1-13. 
10. Weber, C.; Noels, H. Atherosclerosis: current pathogenesis and therapeutic options. Nat. Med. 2011, 17, 1410-1422.

11. Minelli, S.; Minelli, P.; Montinari, M. R. Reflections on Atherosclerosis: Lesson from the Past and Future Research Directions. J. Multidiscip. 2020, 13, 621.

12. Hao, W.; Friedman, A. The LDL-HDL profile determines the risk of atherosclerosis: a mathematical model. PLoS One 2014, 9, e90497.

13. Mach, F.; Baigent, C.; Catapano, A. L.; Koskinas, K. C.; Casula, M., et al. 2019 ESC/EAS Guidelines for the management of dyslipidaemias: lipid modification to reduce cardiovascular risk: The Task Force for the management of dyslipidaemias of the European Society of Cardiology (ESC) and European Atherosclerosis Society (EAS). Eur. Heart J. 2019, 41, 111-188.

14. Ference, B. A.; Ginsberg, H. N.; Graham, I.; Ray, K. K.; Packard, C. J., et al. Low-density lipoproteins cause atherosclerotic cardiovascular disease. 1. Evidence from genetic, epidemiologic, and clinical studies. A consensus statement from the European Atherosclerosis Society Consensus Panel. Eur Heart J 2017, 38, 2459-2472.

15. Boren, J.; Chapman, M. J.; Krauss, R. M.; Packard, C. J.; Bentzon, J. F., et al. Low-density lipoproteins cause atherosclerotic cardiovascular disease: pathophysiological, genetic, and therapeutic insights: a consensus statement from the European Atherosclerosis Society Consensus Panel. Eur Heart J 2020.

16. Silverman, M. G.; Ference, B. A.; Im, K.; Wiviott, S. D.; Giugliano, R. P.; Grundy, S. M.; Braunwald, E.; Sabatine, M. S. Association Between Lowering LDL-C and Cardiovascular Risk Reduction Among Different Therapeutic Interventions: A Systematic Review and Meta-analysis. JAMA 2016, 316, 1289-1297.

17. Linsel-Nitschke, P.; Tall, A. R. HDL as a target in the treatment of atherosclerotic cardiovascular disease. Nat. Rev. Drug Discov. 2005, 4, 193-205.

18. Barter, P.; Gotto, A. M.; LaRosa, J. C.; Maroni, J.; Szarek, M.; Grundy, S. M.; Kastelein, J. J.; Bittner, V.; Fruchart, J.-C. HDL cholesterol, very low levels of LDL cholesterol, and cardiovascular events. N. Engl. J. Med. 2007, 357, 1301-1310.

19. Hafiane, A.; Genest, J. HDL, atherosclerosis, and emerging therapies. Cholesterol 2013, 2013.

20. März, W.; Kleber, M. E.; Scharnagl, H.; Speer, T.; Zewinger, S.; Ritsch, A.; Parhofer, K. G.; von Eckardstein, A.; Landmesser, U.; Laufs, U. HDL cholesterol: reappraisal of its clinical relevance. Clin. Res. Cardiol. 2017, 106, 663-675.

21. Calabresi, L.; Gomaraschi, M.; Simonelli, S.; Bernini, F.; Franceschini, G. HDL and atherosclerosis: Insights from inherited HDL disorders. Biochim. Biophys. Acta. 2015, 1851, 13-18.

22. Lewis, G. F.; Rader, D. J. New insights into the regulation of HDL metabolism and reverse cholesterol transport. Circ. Res. 2005, 96, 1221-1232.

23. Feingold, K. R.; Grunfeld, C.: Introduction to lipids and lipoproteins. In endotext [internet]; MDText. com, 2018.

24. Bostan, M.; Avni, U. H.; Yildirmip, S.; Malkoç, M.; Atak, M.; Demir, A.; Yilmaz, A.; Uðurlu, Y.; Karadað, Z.; Duman, H.; Patiroðlu, Ö. Pleiotropic eff ects of HDL subfractions and HDL-associated enzymes on protection against coronary artery disease. Acta Cardiol. 2015, 70, 333-340.

25. Kosmas, C. E.; Martinez, I.; Sourlas, A.; Bouza, K. V.; Campos, F. N.; Torres, V.; Montan, P. D.; Guzman, E. High-density lipoprotein (HDL) functionality and its relevance to atherosclerotic cardiovascular disease. Drugs context. 2018, 7. 
26. Rosenson, R. S.; Brewer Jr, H. B.; Davidson, W. S.; Fayad, Z. A.; Fuster, V.; Goldstein, J.; Hellerstein, M.; Jiang, X.-C.; Phillips, M. C.; Rader, D. J. Cholesterol efflux and atheroprotection: advancing the concept of reverse cholesterol transport. Circulation. 2012, 125, 1905-1919.

27. Ansell, B. J.; Watson, K. E.; Fogelman, A. M.; Navab, M.; Fonarow, G. C. High-density lipoprotein function: recent advances. J. Am. Coll. Cardiol. 2005, 46, 1792-1798.

28. Bounafaa, A.; Berrougui, H.; Ikhlef, S.; Essamadi, A.; Nasser, B.; Bennis, A.; Yamoul, N.; Ghalim, N.; Khalil, A. Alteration of HDL functionality and PON1 activities in acute coronary syndrome patients. Clin. Biochem. 2014, 47, 318-325.

29. Hung, S.-Y.; Liou, H.-H.; Ger, L.-P.; Chen, L.-K.; Liu, M.-C.; Chung, H.-M.; Chou, K.-J.; Chen, T.H.; Lin, C.-K.; Yang, Y. Clustering of unconventional cardiovascular risk factors among Taiwanese hemodialysis patients. Am. J. Nephrol. 2009, 30, 222-231.

30. Katulanda, G. W.; Katulanda, P.; Adler, A. I.; Peiris, S. R.; Draisey, I.; Wijeratne, S.; Sheriff, R.; Matthews, D. R.; Shine, B. Apolipoproteins in diabetes dyslipidaemia in South Asians with young adult-onset diabetes: distribution, associations and patterns. Ann. Clin. Biochem. 2010, 47, 29-34.

31. Di Bartolo, B. A.; Scherer, D. J.; Nicholls, S. J. Inducing apolipoprotein AI synthesis to reduce cardiovascular risk: from ASSERT to SUSTAIN and beyond. Archives of medical science: AMS 2016, 12, 1302.

32. Stoffel, W.; Krüger, E.; Deutzmann, R. Cell-Free Translation of Human Liver Apolipoprotein AI and All mRNA Processing of Primary Translation Products. Hoppe-Seyler's Zeitschrift für physiologische Chemie 1983, 364, 227-238.

33. Chau, P.; Fielding, P. E.; Fielding, C. J. Bone morphogenetic protein-1 (BMP-1) cleaves human proapolipoprotein A1 and regulates its activation for lipid binding. Biochemistry. 2007, 46, 84458450.

34. Zhu, J.; Gardner, J.; Pullinger, C. R.; Kane, J. P.; Thompson, J. F.; Francone, O. L. Regulation of apoAI processing by procollagen C-proteinase enhancer-2 and bone morphogenetic protein-1. J. Lipid Res. 2009, 50, 1330-1339.

35. Rye, K.-A.; Barter, P. J. Cardioprotective functions of HDLs. J. Lipid Res. 2014, 55, 168-179.

36. Basso, F.; Freeman, L.; Knapper, C. L.; Remaley, A.; Stonik, J.; Neufeld, E. B.; Tansey, T.; Amar, M. J.; Fruchart-Najib, J.; Duverger, N. Role of the hepatic ABCA1 transporter in modulating intrahepatic cholesterol and plasma HDL cholesterol concentrations. J. Lipid Res. 2003, 44, 296-302.

37. Badimon, J. J.; Badimon, L.; Fuster, V. Regression of atherosclerotic lesions by high density lipoprotein plasma fraction in the cholesterol-fed rabbit. J. Clin. Invest. 1990, 85, 1234-1241.

38. Shah, P. K.; Nilsson, J.; Kaul, S.; Fishbein, M. C.; Ageland, H.; Hamsten, A.; Johansson, J.; Karpe, F.; Cercek, B. Effects of recombinant apolipoprotein A-I(Milano) on aortic atherosclerosis in apolipoprotein E-deficient mice. Circulation. 1998, 97, 780-785.

39. Zhang, Y.; Zanotti, I.; Reilly, M. P.; Glick, J. M.; Rothblat, G. H.; Rader, D. J. Overexpression of apolipoprotein A-I promotes reverse transport of cholesterol from macrophages to feces in vivo. Circulation. 2003, 108, 661-663.

40. Barter, P. J.; Nicholls, S.; Rye, K. A.; Anantharamaiah, G. M.; Navab, M.; Fogelman, A. M. Antiinflammatory properties of HDL. Circ. Res. 2004, 95, 764-772.

41. Dimayuga, P.; Zhu, J.; Oguchi, S.; Chyu, K. Y.; Xu, X. O.; Yano, J.; Shah, P. K.; Nilsson, J.; Cercek, B. Reconstituted HDL containing human apolipoprotein A-1 reduces VCAM-1 expression and neointima formation following periadventitial cuff-induced carotid injury in apoE null mice. Biochem. Biophys. Res. Commun. 1999, 264, 465-468. 
42. Degoma, E. M.; Rader, D. J. Novel HDL-directed pharmacotherapeutic strategies. Nat. Rev. Cardiol. 2011, 8, 266.

43. Nissen, S. E.; Tsunoda, T.; Tuzcu, E. M.; Schoenhagen, P.; Cooper, C. J.; Yasin, M.; Eaton, G. M.; Lauer, M. A.; Sheldon, W. S.; Grines, C. L.; Halpern, S.; Crowe, T.; Blankenship, J. C.; Kerensky, R. Effect of Recombinant ApoA-I Milano on Coronary Atherosclerosis in Patients With Acute Coronary Syndromes: A Randomized Controlled Trial. JAMA 2003, 290, 2292-2300.

44. Nissen, S. E.; Tsunoda, T.; Tuzcu, E. M.; Schoenhagen, P.; Cooper, C. J.; Yasin, M.; Eaton, G. M.; Lauer, M. A.; Sheldon, W. S.; Grines, C. L.; Halpern, S.; Crowe, T.; Blankenship, J. C.; Kerensky, R. Effect of recombinant ApoA-I Milano on coronary atherosclerosis in patients with acute coronary syndromes: a randomized controlled trial. JAMA 2003, 290, 2292-2300.

45. Tardif, J. C.; Grégoire, J.; L’Allier, P. L.; Ibrahim, R.; Lespérance, J.; Heinonen, T. M.; Kouz, S.; Berry, C.; Basser, R.; Lavoie, M. A.; Guertin, M. C.; Rodés-Cabau, J. Effects of reconstituted high-density lipoprotein infusions on coronary atherosclerosis: a randomized controlled trial. JAMA 2007, 297, 1675-1682.

46. Bailey, D.; Jahagirdar, R.; Gordon, A.; Hafiane, A.; Campbell, S.; Chatur, S.; Wagner, G. S.; Hansen, H. C.; Chiacchia, F. S.; Johansson, J.; Krimbou, L.; Wong, N. C.; Genest, J. RVX-208: a small molecule that increases apolipoprotein A-I and high-density lipoprotein cholesterol in vitro and in vivo. J. Am. Coll. Cardiol. 2010, 55, 2580-2589.

47. Navab, M.; Anantharamaiah, G.; Reddy, S. T.; Hama, S.; Hough, G.; Grijalva, V. R.; Yu, N.; Ansell, B. J.; Datta, G.; Garber, D. W. Apolipoprotein AI mimetic peptides. Arterioscler. Thromb. Vasc. Biol. 2005, 25, 1325-1331.

48. Islam, R. M.; Pourmousa, M.; Sviridov, D.; Gordon, S. M.; Neufeld, E. B.; Freeman, L. A.; Perrin, B. S.; Pastor, R. W.; Remaley, A. T. Structural properties of apolipoprotein AI mimetic peptides that promote ABCA1-dependent cholesterol efflux. Sci. Rep. 2018, 8, 1-15.

49. Smolders, L.; Plat, J.; Mensink, R. P. Dietary Strategies and Novel Pharmaceutical Approaches Targeting Serum ApoA-I Metabolism: A Systematic Overview. J. Nutr. Metab. 2017, 2017.

50. Dunbar, R. L.; Movva, R.; Bloedon, L. T.; Duffy, D.; Norris, R. B.; Navab, M.; Fogelman, A. M.; Rader, D. J. Oral Apolipoprotein A-I Mimetic D-4F Lowers HDL-Inflammatory Index in HighRisk Patients: A First-in-Human Multiple-Dose, Randomized Controlled Trial. Clin. Transl. Sci. 2017, 10, 455-469.

51. Dullens, S. P.; Plat, J.; Mensink, R. P. Increasing apoA-I production as a target for CHD risk reduction. Nutr Metab Cardiovasc Dis. 2007, 17, 616-628.

52. Mooradian, A. D.; Haas, M. J.; Wong, N. C. Transcriptional control of apolipoprotein AI gene expression in diabetes. Diabetes. 2004, 53, 513-520.

53. Zannis, V. I.; Kan, H.-Y.; Kritis, A.; Zanni, E. E.; Kardassis, D. Transcriptional regulatory mechanisms of the human apolipoprotein genes in vitro and in vivo. Curr. Opin. Lipidol. 2001, 12, 181-207.

54. Jin, F.-Y.; Kamanna, V. S.; Chuang, M.-Y.; Morgan, K.; Kashyap, M. L. Gemfibrozil stimulates apolipoprotein AI synthesis and secretion by stabilization of mRNA transcripts in human hepatoblastoma cell line (Hep G2). Arterioscler. Thromb. Vasc. Biol. 1996, 16, 1052-1062.

55. Arakawa, R.; Tamehiro, N.; Nishimaki-Mogami, T.; Ueda, K.; Yokoyama, S. Fenofibric acid, an active form of fenofibrate, increases apolipoprotein AI-mediated high-density lipoprotein biogenesis by enhancing transcription of ATP-binding cassette transporter A1 gene in a liver X receptor-dependent manner. Arterioscler. Thromb. Vasc. Biol. 2005, 25, 1193-1197. 
56. McLure, K. G.; Gesner, E. M.; Tsujikawa, L.; Kharenko, O. A.; Attwell, S.; Campeau, E.; Wasiak, S.; Stein, A.; White, A.; Fontano, E. RVX-208, an inducer of ApoA-I in humans, is a BET bromodomain antagonist. PLoS One 2013, 8, e83190.

57. Kempen, H. J.; Bellus, D.; Fedorov, O.; Nicklisch, S.; Filippakopoulos, P.; Picaud, S.; Knapp, S.: Stimulation of hepatic apolipoprotein AI production by novel thieno-triazolodiazepines: roles of the classical benzodiazepine receptor, PAF receptor, and bromodomain binding. SAGE Publications Sage UK: London, England, 2013.

58. Hargrove, G.; Junco, A.; Wong, N. Hormonal regulation of apolipoprotein AI. J. Mol. Endocrinol. 1999, 22 2, 103-111.

59. Duez, H.; Lefebvre, B.; Poulain, P.; Torra, I. P.; Percevault, F.; Luc, G.; Peters, J. M.; Gonzalez, F. J.; Gineste, R.; Helleboid, S. Regulation of human ApoA-I by gemfibrozil and fenofibrate through selective peroxisome proliferator-activated receptor a modulation. Arterioscler. Thromb. Vasc. Biol. 2005, 25, 585-591.

60. Tzameli, I.; Zannis, V. I. Binding specificity and modulation of the ApoA-I promoter activity by homo-and heterodimers of nuclear receptors. J. Biol. Chem. 1996, 271, 8402-8415.

61. Vu-Dac, N.; Schoonjans, K.; Laine, B.; Fruchart, J.-C.; Auwerx, J.; Staels, B. Negative regulation of the human apolipoprotein AI promoter by fibrates can be attenuated by the interaction of the peroxisome proliferator-activated receptor with its response element. J. Biol. Chem. 1994, 269, 31012-31018.

62. Kan, H.-Y.; Georgopoulos, S.; Zanni, M.; Shkodrani, A.; Tzatsos, A.; Xie, H. X.; Zannis, V. I. Contribution of the hormone-response elements of the proximal ApoA-I promoter, ApoCIII enhancer, and C/EBP binding site of the proximal ApoA-I promoter to the hepatic and intestinal expression of the ApoA-I and ApoCIII genes in transgenic mice. Biochemistry. 2004, 43, 50845093.

63. Harnish, D. C.; Malik, S.; Karathanasis, S. K. Activation of apolipoprotein AI gene transcription by the liver-enriched factor HNF-3. J. Biol. Chem. 1994, 269, 28220-28226.

64. Lam, J. K.; Matsubara, S.; Mihara, K.; Zheng, X.-1.; Mooradian, A. D.; Wong, N. C. Insulin induction of apolipoprotein AI, role of Sp1. Biochemistry. 2003, 42, 2680-2690.

65. Esteve, E.; Ricart, W.; Fernández-Real, J. M. Dyslipidemia and inflammation: an evolutionary conserved mechanism. Clin. Nutr. 2005, 24, 16-31.

66. Kosmas, C. E.; Silverio, D.; Sourlas, A.; Montan, P. D.; Guzman, E. Dysfunctional high-density lipoprotein and atherogenesis. Vessel Plus. 2019, 3, 2.

67. Navab, M.; Anantharamaiah, G.; Fogelman, A. M. The role of high-density lipoprotein in inflammation. Trends Cardiovasc. Med. 2005, 15, 158-161.

68. Wigmore, S. J.; Fearon, K. C.; Maingay, J. P.; Lai, P. B.; Ross, J. A. Interleukin-8 can mediate acutephase protein production by isolated human hepatocytes. AM J Physiol-endoc M. 1997, 273, E720-E726.

69. Cabana, V. G.; Siegel, J. N.; Sabesin, S. M. Effects of the acute phase response on the concentration and density distribution of plasma lipids and apolipoproteins. J. Lipid Res. 1989, 30, 39-49.

70. Van Leeuwen, H. J.; Heezius, E. C.; Dallinga, G. M.; van Strijp, J. A.; Verhoef, J.; van Kessel, K. P. Lipoprotein metabolism in patients with severe sepsis. Crit. Care Med. 2003, 31, 1359-1366.

71. Van Lenten, B. J.; Hama, S. Y.; de Beer, F. C.; Stafforini, D. M.; McIntyre, T. M.; Prescott, S. M.; La Du, B. N.; Fogelman, A. M.; Navab, M. Anti-inflammatory HDL becomes pro-inflammatory during the acute phase response. Loss of protective effect of HDL against LDL oxidation in aortic wall cell cocultures. J. Clin. Invest. 1995, 96, 2758-2767. 
72. Gurung, M.; Li, Z.; You, H.; Rodrigues, R.; Jump, D. B.; Morgun, A.; Shulzhenko, N. Role of gut microbiota in type 2 diabetes pathophysiology. EBioMedicine. 2020, 51, 102590.

73. Hu, G.-X.; Chen, G.-R.; Xu, H.; Ge, R.-S.; Lin, J. Activation of the AMP activated protein kinase by short-chain fatty acids is the main mechanism underlying the beneficial effect of a high fiber diet on the metabolic syndrome. Med. Hypotheses 2010, 74, 123-126.

74. Sun, M.; Wu, W.; Liu, Z.; Cong, Y. Microbiota metabolite short chain fatty acids, GPCR, and inflammatory bowel diseases. J. Gastroenterol. 2017, 52, 1-8.

75. Tungland, B.: Human Microbiota in Health and Disease: From Pathogenesis to Therapy; Academic press, 2018.

76. Canfora, E. E.; Jocken, J. W.; Blaak, E. E. Short-chain fatty acids in control of body weight and insulin sensitivity. Nat. Rev. Endocrinol. 2015, 11, 577.

77. Den Besten, G.; van Eunen, K.; Groen, A. K.; Venema, K.; Reijngoud, D.-J.; Bakker, B. M. The role of short-chain fatty acids in the interplay between diet, gut microbiota, and host energy metabolism. J. Lipid Res. 2013, 54, 2325-2340.

78. Byrne, C.; Chambers, E.; Morrison, D.; Frost, G. The role of short chain fatty acids in appetite regulation and energy homeostasis. Int. J. Obes. 2015, 39, 1331-1338.

79. Wong, J. M.; De Souza, R.; Kendall, C. W.; Emam, A.; Jenkins, D. J. Colonic health: fermentation and short chain fatty acids. J. Clin. Gastroenterol. 2006, 40, 235-243.

80. Cummings, J.; Pomare, E.; Branch, W.; Naylor, C.; Macfarlane, G. Short chain fatty acids in human large intestine, portal, hepatic and venous blood. Gut. 1987, 28, 1221-1227.

81. Moschen, I.; Bröer, A.; Galić, S.; Lang, F.; Bröer, S. Significance of short chain fatty acid transport by members of the monocarboxylate transporter family (MCT). Neurochem. Res. 2012, 37, 25622568.

82. Ritzhaupt, A.; Wood, I. S.; Ellis, A.; Hosie, K. B.; Shirazi-Beechey, S. P. Identification and characterization of a monocarboxylate transporter (MCT1) in pig and human colon: its potential to transport l-lactate as well as butyrate. J. Physiol. 1998, 513, 719-732.

83. Bloemen, J. G.; Venema, K.; van de Poll, M. C.; Damink, S. W. O.; Buurman, W. A.; Dejong, C. $\mathrm{H}$. Short chain fatty acids exchange across the gut and liver in humans measured at surgery. Clin. Nutr. 2009, 28, 657-661.

84. Layden, B. T.; Angueira, A. R.; Brodsky, M.; Durai, V.; Lowe Jr, W. L. Short chain fatty acids and their receptors: new metabolic targets. Transl Res. 2013, 161, 131-140.

85. Brown, A. J.; Goldsworthy, S. M.; Barnes, A. A.; Eilert, M. M.; Tcheang, L.; Daniels, D.; Muir, A. I.; Wigglesworth, M. J.; Kinghorn, I.; Fraser, N. J. The Orphan G protein-coupled receptors GPR41 and GPR43 are activated by propionate and other short chain carboxylic acids. J. Biol. Chem. 2003, 278, 11312-11319.

86. Alexander, C.; Swanson, K. S.; Fahey Jr, G. C.; Garleb, K. A. Perspective: physiologic importance of short-chain fatty acids from nondigestible carbohydrate fermentation. Adv. Nutr. 2019, 10, 576589.

87. Koh, A.; De Vadder, F.; Kovatcheva-Datchary, P.; Bäckhed, F. From Dietary Fiber to Host Physiology: Short-Chain Fatty Acids as Key Bacterial Metabolites. Cell. 2016, 165, 1332-1345.

88. Topping, D. L.; Clifton, P. M. Short-chain fatty acids and human colonic function: roles of resistant starch and nonstarch polysaccharides. Physiol. Rev. 2001, 81, 1031-1064.

89. Sanna, S.; van Zuydam, N. R.; Mahajan, A.; Kurilshikov, A.; Vila, A. V.; Võsa, U.; Mujagic, Z.; Masclee, A. A.; Jonkers, D. M.; Oosting, M. Causal relationships among the gut microbiome, short-chain fatty acids and metabolic diseases. Nat. Genet. 2019, 51, 600-605. 
90. Cornejo-Pareja, I.; Muñoz-Garach, A.; Clemente-Postigo, M.; Tinahones, F. J. Importance of gut microbiota in obesity. Eur. J. Clin. Nutr. 2019, 72, 26-37.

91. Canfora, E. E.; van der Beek, C. M.; Jocken, J. W. E.; Goossens, G. H.; Holst, J. J.; Olde Damink, S. W. M.; Lenaerts, K.; Dejong, C. H. C.; Blaak, E. E. Colonic infusions of short-chain fatty acid mixtures promote energy metabolism in overweight/obese men: a randomized crossover trial. Sci. Rep. 2017, 7, 2360.

92. Chambers, E. S.; Preston, T.; Frost, G.; Morrison, D. J. Role of gut microbiota-generated shortchain fatty acids in metabolic and cardiovascular health. Curr. Nutr. Rep. 2018, 7, 198-206.

93. Li, M.; van Esch, B. C.; Wagenaar, G. T.; Garssen, J.; Folkerts, G.; Henricks, P. A. Pro-and antiinflammatory effects of short chain fatty acids on immune and endothelial cells. Eur. J. Pharmacol. 2018, 831, 52-59.

94. Ohira, H.; Tsutsui, W.; Fujioka, Y. Are short chain fatty acids in gut microbiota defensive players for inflammation and atherosclerosis? J Atheroscler Thromb. 2017, 24, 660-672.

95. Thibault, R.; Blachier, F.; Darcy-Vrillon, B.; De Coppet, P.; Bourreille, A.; Segain, J.-P. Butyrate utilization by the colonic mucosa in inflammatory bowel diseases: a transport deficiency. Inflamm. Bowel Dis. 2010, 16, 684-695.

96. Bartolomaeus, H.; Balogh, A.; Yakoub, M.; Homann, S., et al. Short-Chain Fatty Acid Propionate Protects From Hypertensive Cardiovascular Damage. Circulation. 2019, 139, 1407-1421.

97. Den Besten, G.; Bleeker, A.; Gerding, A.; van Eunen, K.; Havinga, R.; van Dijk, T. H.; Oosterveer, M. H.; Jonker, J. W.; Groen, A. K.; Reijngoud, D. J.; Bakker, B. M. Short-Chain Fatty Acids Protect Against High-Fat Diet-Induced Obesity via a PPAR $\gamma$-Dependent Switch From Lipogenesis to Fat Oxidation. Diabetes. 2015, 64, 2398-2408.

98. Roshanravan, N.; Mahdavi, R.; Alizadeh, E.; Jafarabadi, M. A.; Hedayati, M.; Ghavami, A.; Alipour, S.; Alamdari, N. M.; Barati, M.; Ostadrahimi, A. Effect of Butyrate and Inulin Supplementation on Glycemic Status, Lipid Profile and Glucagon-Like Peptide 1 Level in Patients with Type 2 Diabetes: A Randomized Double-Blind, Placebo-Controlled Trial. Horm. Metab. Res. 2017, 49, 886-891.

99. Chambers, E. S.; Byrne, C. S.; Rugyendo, A.; Morrison, D. J.; Preston, T.; Tedford, C.; Bell, J. D.; Thomas, L.; Akbar, A. N.; Riddell, N. E.; Sharma, R.; Thursz, M. R.; Manousou, P.; Frost, G. The effects of dietary supplementation with inulin and inulin-propionate ester on hepatic steatosis in adults with non-alcoholic fatty liver disease. Diabetes Obes. Metab. 2019, 21, 372-376.

100. Reijnders, D.; Goossens, G. H.; Hermes, G. D.; Neis, E. P.; van der Beek, C. M.; Most, J.; Holst, J. J.; Lenaerts, K.; Kootte, R. S.; Nieuwdorp, M. Effects of gut microbiota manipulation by antibiotics on host metabolism in obese humans: a randomized double-blind placebo-controlled trial. Cell Metab. 2016, 24, 63-74.

101. De Smet, E. Plant stanol esters: focus on intestinal lipoprotein metabolism. 2014.

102. Holota, Y.; Dovbynchuk, T.; Kaji, I.; Vareniuk, I.; Dzyubenko, N.; Chervinska, T.; Zakordonets, L.; Stetska, V.; Ostapchenko, L.; Serhiychuk, T. The long-term consequences of antibiotic therapy: Role of colonic short-chain fatty acids (SCFA) system and intestinal barrier integrity. PLoS One 2019, 14, e0220642.

103. Zhao, Y.; Liu, J.; Hao, W.; Zhu, H.; Liang, N.; He, Z.; Ma, K. Y.; Chen, Z.-Y. Structure-specific effects of short-chain fatty acids on plasma cholesterol concentration in male syrian hamsters. J. Agric. Food Chem. 2017, 65, 10984-10992.

104. Popeijus, H. E.; van Otterdijk, S. D.; van der Krieken, S. E.; Konings, M.; Serbonij, K.; Plat, J.; Mensink, R. P. Fatty acid chain length and saturation influences PPARa transcriptional activation and repression in HepG2 cells. Mol. Nutr. Food Res. 2014, 58, 2342-2349. 
105. Vu-Dac, N.; Chopin-Delannoy, S.; Gervois, P.; Bonnelye, E.; Martin, G.; Fruchart, J.-C.; Laudet, V.; Staels, B. The nuclear receptors peroxisome proliferator-activated receptor $\alpha$ and Rev-erba mediate the species-specific regulation of apolipoprotein AI expression by fibrates. J. Biol. Chem. 1998, 273, 25713-25720.

106. Schnuck, J. K.; Sunderland, K. L.; Gannon, N. P.; Kuennen, M. R.; Vaughan, R. A. Leucine Stimulates Peroxisome Proliferator Activator Receptors in Skeletal Muscle: 1603 Board\# 256 June 2, 800 AM-930 AM. Med. Sci. Sports Exerc. 2016, 48, 441-442.

107. Van Meijl, L. E.; Popeijus, H. E.; Mensink, R. P. Amino acids stimulate Akt phosphorylation, and reduce IL-8 production and NF- $\kappa$ B activity in HepG2 liver cells. Mol. Nutr. Food Res. 2010, 54, 1568-1573.

108. Zhang, N.; Chu, E. S.; Zhang, J.; Li, X.; Liang, Q.; Chen, J.; Chen, M.; Teoh, N.; Farrell, G.; Sung, J. J.; Yu, J. Peroxisome proliferator activated receptor alpha inhibits hepatocarcinogenesis through mediating NF-KB signaling pathway. Oncotarget. 2014, 5, 8330-8340. 


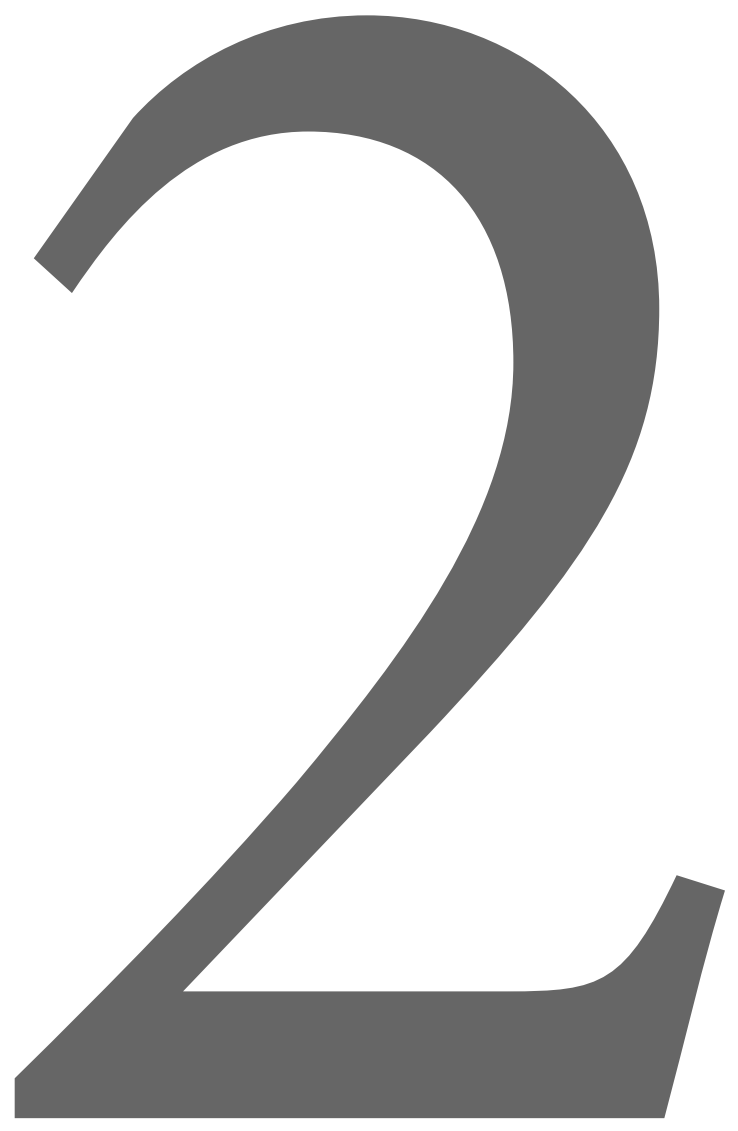




\section{The effects of short-chain fatty acids on the transcription and secretion of apolipoprotein A-I in human hepatocytes in vitro}

Jehad Z. Tayyeb | Herman E. Popeijus | Ronald P. Mensink | Maurice C.J.M. Konings | Kim H.R. Mulders | Jogchum Plat 


\section{Abstract}

Apolipoprotein-I (ApoA-I), the major component of high-density lipoprotein (HDL) particles, mediates cholesterol efflux by which it facilitates the removal of excess cholesterol from peripheral tissues. Therefore, elevating ApoA-I production leading to the production of new pre- $\beta$-HDL particles is thought to be beneficial in the prevention of cardiovascular diseases. Recently, we observed that amoxicillin treatment led to decreased HDL concentrations in healthy human volunteers. We questioned whether this antibiotic effect was directly or indirectly, via changed shortchain fatty acids (SCFA) concentrations through an altered gut microflora. Therefore, we here evaluated the effects of amoxicillin and various SCFA on hepatic ApoA-I expression, secretion, and the putative underlying pathways. Human hepatocytes (HepG2) were exposed to increasing dose of amoxicillin or SCFA for 48 hours. ApoA-I messenger RNA (mRNA) transcription and secreted protein were analyzed using quantitative polymerase chain reaction and enzyme-linked immunosorbent assay, respectively. To study underlying mechanisms, changes in mRNA expression of KEAP1, CPT1, and PPARa, as well as a PPARa transactivation assay, were analyzed. Amoxicillin dose-dependently decreased ApoA-I mRNA transcription as well as ApoA-I protein secretion. SCFA treatment resulted in a dose-dependent stimulation of ApoA-I mRNA transcription, however, the ApoA-I protein secretion was decreased. Furthermore, SCFA treatment increased PPAR $\alpha$ transactivation, PPAR $\alpha$ and CPT1 mRNA transcription, whereas KEAP1 mRNA transcription was decreased. The results found indicate that direct treatment of HepG2 cells with amoxicillin has either direct effects on lowering ApoA-I transcription and secretion or indirect effects via modified SCFA concentrations because SCFA were found to stimulate hepatic ApoA-I expression. Furthermore, BET inhibition and PPARa activation were identified as possible mechanisms behind the observed effects on ApoA-I transcription. 


\section{Introduction}

Several studies have suggested that antibiotics affect lipid and lipoprotein metabolism $(1,2)$. In fact, in a recent placebo-controlled trial we observed that amoxicillin treatment for 7 days lowered high-density lipoprotein (HDL) cholesterol concentrations in healthy volunteers (2). Whether this decrease is harmful as related to cardiovascular diseases (CVDs)-risk, is questionable because HDL functionality may be more important in this respect than HDL cholesterol concentrations (3). These functional, antiatherogenic effects have been mainly attributed to its most predominant structural protein particle, apolipoprotein-I (ApoA-I), which is produced by the small intestine and the liver (4). Amongst others, ApoA-I mediates cholesterol efflux facilitating the removal of excess cholesterol from peripheral tissues and the uptake by the liver for excretion into bile (5). Since ApoA-I is one of the most important HDL proteins in terms of functionality, a better understanding of ApoA-I transcription and production is warranted. Therefore, we explored in vitro the potential antibiotic-related effects on ApoA-I transcription and synthesis. Effects of antibiotics on de novo ApoA-I expression can be a direct effect of the antibiotic on gene transcription or an indirect effect by affecting microbiota composition. Effects of antibiotics on ApoA-I transcription have to the best of our knowledge never been studied. Regarding indirect effects, Reijnders et al. (1) have recently shown that the decreased bacterial diversity after the intake of vancomycin resulted in reduced circulating short-chain fatty acids (SCFA) concentrations. These SCFA are produced in the colon by the fermentation of dietary fibers such as resistant starches and non-starch polysaccharides (6). SCFA have been linked to multiple beneficial health effects including a reduced risk of inflammatory diseases, gastrointestinal disorders, cancer, and CVD (7). Furthermore, it was recently shown that dietary SCFA increase HDL cholesterol concentrations in hamsters (8). We therefore hypothesized that antibiotics directly, or indirectly via reduced SCFA concentrations, may relate to the changes in ApoA-I transcription, which eventually led to decreased HDL cholesterol concentrations.

Besides evaluating the effects of SCFA on hepatic ApoA-I expression, an additional question is how these potential effects are regulated at a molecular level. Previous studies reported that both bromodomain and extra-terminal domain (BET) inhibition and PPARa activation had a major effect on ApoA-I transcription $(9,10)$. For example, BET protein inhibitors such as JQ1(+) increased ApoA-I expression and protein secretion in human hepatocellular liver carcinoma (HepG2) cells $(11,12)$. BET inhibitors can bind to BET proteins such as $\mathrm{BRD} 4$, a general transcriptional regulator, which can regulate transcription of target genes such as KEAP1 $(13,14)$. PPARa is a nuclear receptor that forms a heterodimer with the retinoid $\mathrm{X}$ receptor, which then binds to specific response elements (PPREs) within promoter regions of target genes such as PPARa itself, CPT1 (15) and ApoA-I (16). Various 
dietary components such as long-chain fatty acids have been recognized as natural ligands for PPARa (17) but there are indications that SCFA may have similar effects $(18,19)$. Therefore, except for effects on changes in ApoA-I messenger RNA (mRNA), we also evaluated changes in KEAP1, CPT1 and PPAR $a$ mRNA expressions during exposure of HepG2 cells to different SCFA to examine potential underlying pathways.

\section{Material and methods}

\section{Materials}

Human hepatocellular liver carcinoma (HepG2) cells were kindly provided by Sten BraeschAndersen (Mabtech, Nacka Strand, Sweden). Cell culture flasks and plates were obtained from Corning (Cambridge, USA). Minimum Essential Medium (MEM), sodium pyruvate, non-essential amino acids (NEAA) and penicillin and streptomycin were all obtained from Thermo Fisher Scientific (Bleiswijk, Netherlands). Fetal bovine serum (FBS) was purchased from PAA (Toronto, Canada). Amoxicillin was obtained from Sigma (Uithoorn, Netherlands). Propionic acid (C3), butyric acid (C4), valeric acid (C5) and hexanoic acid (C6) were purchased from Sigma (Uithoorn, Netherlands). The BET inhibitor JQ1(+), was purchased from Bio-techne - R\&D (Minneapolis, USA). Thapsigargin (Taps), an ER-stress inducer, was purchased from Sigma (Uithoorn, Netherlands). DMSO and Tri-reagent were obtained from Sigma (Uithoorn, Netherlands).

\section{Cell culture and SCFA treatment}

HepG2 cells were cultured at $37^{\circ} \mathrm{C}$ in a humidified atmosphere of $5 \%$ carbon dioxide $\left(\mathrm{CO}_{2}\right)$ in MEM containing $10 \%$ heat inactivated FBS, $1 \%$ sodium pyruvate, $1 \%$ NEAA and $1 \%$ of penicillin-streptomycin mixture. In the amoxicillin experiments cells were cultured without penicillin-streptomycin mixture. For all experiments, cells were seeded in a 24-well plate at a density of 200,000 cells per well. Cell viability was daily inspected by microscope and when cells reached a density of $80-90 \%$, they were incubated for $48 \mathrm{~h}$ in the medium (MEM without FBS) plus a concentration range of 0-7 mM SCFA (C3, C4, C5 or C6) or amoxicillin $(0-200 \mu \mathrm{g} / \mathrm{ml})$ or $3 \mu \mathrm{M} \mathrm{JQ1}(+)$. The positive control JQ1(+) was included - separate from the SCFA - in all experiments to ensure the cells were responsive and capable to produce sufficient amounts of ApoA-I mRNA. All SCFA as well as JQ1(+) were dissolved in dimethyl sulfoxide (DMSO, cell culture tested) and effects were expressed relative to those of the carrier control (DMSO only). Final DMSO concentration was always $0.2 \%$. As amoxicillin was dissolved in water, effects of amoxicillin were compared to those of a water control. In previous study (20), it was shown that after oral administration of $500 \mathrm{mg}$ 
of amoxicillin (taken three times a day), serum peak levels were between 6.0 to $15.3 \mu \mathrm{g} / \mathrm{ml}$ and after intravenous administration of $500 \mathrm{mg}$ of amoxicillin (taken three times a day), serum peak levels were between 30.1 to $52.1 \mu \mathrm{g} / \mathrm{ml}$. It was therefore decided to test the effect of the antibiotics on HepG2 cells in concentrations of 3, 6, 12.5, 25, 50, 100 and $200 \mu \mathrm{g} / \mathrm{ml}$. Medium was collected for analysis of ApoA-I concentrations and cells were harvested for analysis of mRNA expression after lysing with Tri-reagent. Both medium and lysed cells were snap frozen in liquid nitrogen and stored at $-80{ }^{\circ} \mathrm{C}$ until further analysis.

\section{Quantification of ApoA-I secretion levels in the culture medium}

To investigate ApoA-I secretion by HepG2 cells, ApoA-I protein concentrations in culture medium were measured by an enzyme-linked immunoassay (ELISA) obtained from Mabtech (Nacka Strand, Sweden). Direct sandwich ELISA was performed according the manufacturer's instructions, with slight modifications, blocker BSA 10\% (Thermo Fisher Scientific, Bleiswijk, Netherlands) was added to block buffer and to dilution buffer with final concentration of $1 \%$ and $0.1 \%$ respectively.

\section{Quantification of gene mRNA transcription}

To evaluate mRNA expression levels of ApoA-I, CPT1, KEAP1 and PPARa, total RNA was isolated using Tri-reagent, according the manufacturer's instructions. The RNeasy mini kit (Qiagen, Hilden, Germany) was used for RNA purification. For cDNA synthesis, 350 ng of total RNA was reverse-transcribed using RNAse inhibitor, dNTP's, random hexamers, MMLV reverse trans, DTT and 5xFS buffer (Thermo Fisher Scientific, Bleiswijk, Netherlands). The resulting cDNA was used for real time quantitative PCR using TaqMan Gene Expression Assays using Cyclophilin A (Hs99999904) as a housekeeping control. To quantify ApoA-I, KEAP1, PPAR $\alpha$ and CPT1, the following TaqMan Genes Expression Assays Hs00163641, Hs00202227, Hs00231882 and Hs00912671 were used. Values are presented as relative gene expressions based on the $\mathrm{Ct}$ values, normalized for the internal control Cyclophilin A and compared to the control conditions.

\section{Luciferase assay}

To investigate PPARa transcriptional activity, HepG2 cells were transfected with X-treme gene 9 DNA transfection reagent (Sigma, Uithoorn, Netherlands) and the following plasmids: pcDNA3.1, pcDNA3.1_PPARa, pGL3 and pGL3_PPRE as previously described (17). Following transfection and $48 \mathrm{~h}$ SCFA treatment, cells were lysed in luciferase lysis buffer (Promega, Madison, USA), and measured for luciferase activity, reflecting 
PPARa transactivation, using a GloMax 96 Microplate luminometer, all according to the manufacturer's manual (Promega, Madison, USA).

\section{Statistical analysis}

All experiments were performed in Duplo and each experiment was repeated three times. To test the dose-response relationship with the gene of interest, a regression analysis was performed. When the analysis was not-linear, quadratic polynomial regression was performed. For all statistical analysis, the regression coefficient was considered statistically significant at $\mathrm{p}<0.05$. Statistical analysis was performed using SPSS v.25 (IBM Corp., Armonk, NY, USA).

\section{Results}

\section{Effects of amoxicillin on ApoA-I mRNA expression and protein secretion}

Amoxicillin significantly $(\mathrm{p}<0.05)$ and dose-dependently decreased ApoA-I mRNA expression up to $30 \%$. Interestingly, the lower ApoA-I mRNA expression translated into a lower ApoA-I protein secretion $(\mathrm{p}<0.01)$ into the culture medium, i.e. ApoA-I protein concentrations followed mRNA expression. As expected, JQ1(+), a well-defined BET inhibitor, which was used as positive control, increased ApoA-I mRNA expression as well as ApoA-I protein secretion, while the negative control thapsigargin, an ER-stress inducer, inhibited both ApoA-I mRNA expression and protein secretion (Figure 2.1). Altogether, these findings suggest direct inhibitory effects of amoxicillin on ApoA-I transcription and secretion.

\section{Effect of SCFA on ApoA-I mRNA expression and protein secretion}

The positive control JQ1(+) strongly increased ApoA-I mRNA expression in all experiments with SCFA (Figure 2.2). This indicated that the cells were responsive and allows further analysis with SCFA. ApoA-I mRNA expression dose-dependently increased after C3 treatment $(\mathrm{p}<0.001)$, with a maximum of a 3.2 -fold increase at $7 \mathrm{mM}$. Treatment with C4 first dose-dependently increased ApoA-I mRNA expression which resulted in a 3-fold increase at $3 \mathrm{mM}$ followed by a dose dependent decrease at higher concentrations down to $\pm 50 \%$ of basal ApoA-I mRNA expression at $7 \mathrm{mM}(\mathrm{p}<0.001)$. C5 dose-dependently and significantly $(\mathrm{p}<0.001)$ increased ApoA-I mRNA expression up to a 3-fold increase at 5.5 mM. C6 gradually increased ApoA-I mRNA expression with a maximum of 2 -fold at $7 \mathrm{mM}$ $(\mathrm{p}<0.01)$ (Figure 2.2). Although ApoA-I mRNA expression was elevated with all types of 

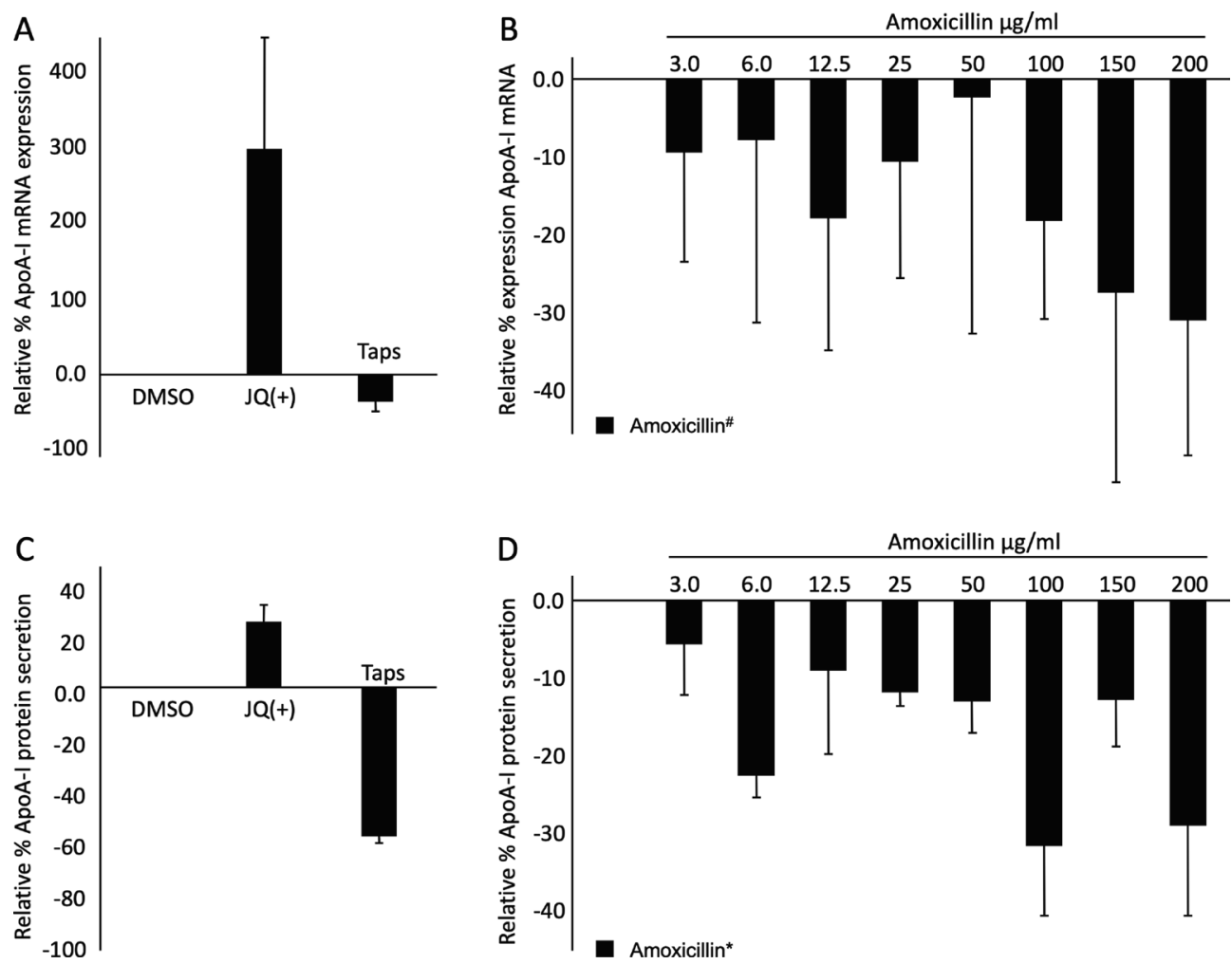

Figure 2.1. (A) Relative ApoA-I mRNA expression in HepG2 cells treated with JQ1(+) $(3 \mu \mathrm{M})$ and Taps $(0.01 \mu \mathrm{M})$. JQ1 $(+)$ was used as a positive control for ApoA-I transcription, whereas Taps was used as a control for reduced ApoA-I transcription. (B) Relative ApoA-I mRNA expression in HepG2 cells treated with different concentrations of amoxicillin. Increasing amoxicillin concentrations show significant regression coefficient deviated from zero $(\mathrm{p}<0.05)$ in ApoA-I mRNA expression. (C) Relative ApoA-I protein secretion by HepG2 cells into the culture medium after treatment with JQ1(+) $(3 \mu \mathrm{M})$ and Taps $(0.01 \mu \mathrm{M})$. JQ1(+) was used as a positive control for ApoA-I protein secretion, whereas Taps was used as a control for reduced ApoA-I protein secretion. (D) Relative ApoA-I protein secretion by HepG2 cells into the culture medium after treatment with different concentrations of amoxicillin. Increasing amoxicillin concentrations show significant regression coefficient deviated from zero $(\mathrm{p}<0.01)$ in ApoA-I protein secretion. All results are presented as the mean, while error bars indicate standard deviations. Data were normalized against expression of the control condition, which was arbitrarily set at $0 \%$. Changes are indicated with ${ }^{*}$ when regression coefficient significantly at $(\mathrm{p}<0.01)$ or with \# when regression coefficient significantly at $(\mathrm{p}<0.05)$. ApoA-I, apolipoprotein-I; DMSO, dimethyl sulfoxide; mRNA, messenger RNA.

SCFA - except higher C4 doses -, we observed for all SCFA a dose-dependent decreases in ApoA-I protein concentrations in the culture medium of the HepG2 cells $(\mathrm{p}<0.05)$ (Figure 2.3). In contrast, the positive control JQ1(+) not only elevated ApoA-I mRNA expression, but also increased ApoA-I protein secretion in the culture medium (Figure 2.3). 


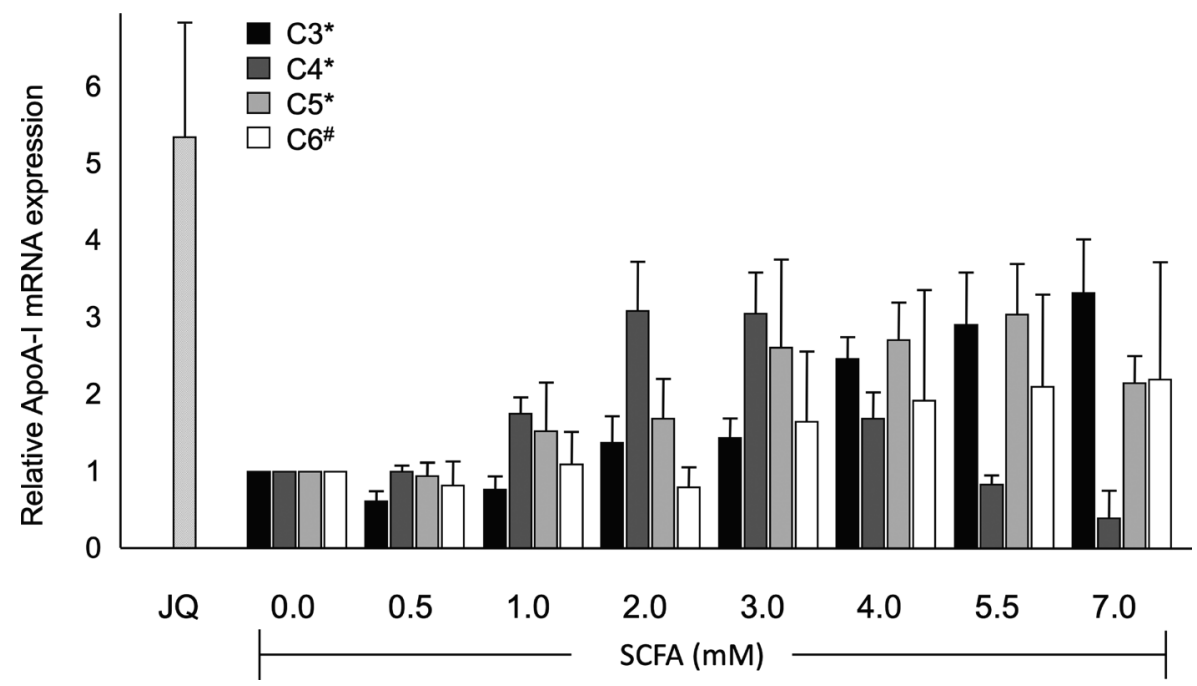

Figure 2.2. Relative ApoA-I mRNA expression in HepG2 cells treated with different concentrations of SCFA. Increasing C3, C4, and C5 concentrations show significant regression coefficient deviated from zero $(\mathrm{p}<0.001)$ in ApoA-I mRNA expression. C6 also show significant regression coefficient deviated from zero $(\mathrm{p}<0.01)$ in ApoA-I mRNA expression. Linear regression for SCFA dose-response effects was performed except C4, quadratic polynomial regression was performed for C4 dose-response effects. JQ1(+) $(3 \mu \mathrm{M})$ was used as a positive control for ApoA-I transcription. All results are presented as the mean, while error bars indicate standard deviations. Data were normalized against expression of the control condition, which was arbitrarily set at 1 . Changes are indicated with ${ }^{*}$ when regression coefficient significantly at $(\mathrm{p}<0.001)$ or with a \# when regression coefficient significantly at $(\mathrm{p}<0.01)$. ApoA-I, apolipoprotein-I; mRNA, messenger RNA; SCFA, short-chain fatty acids.

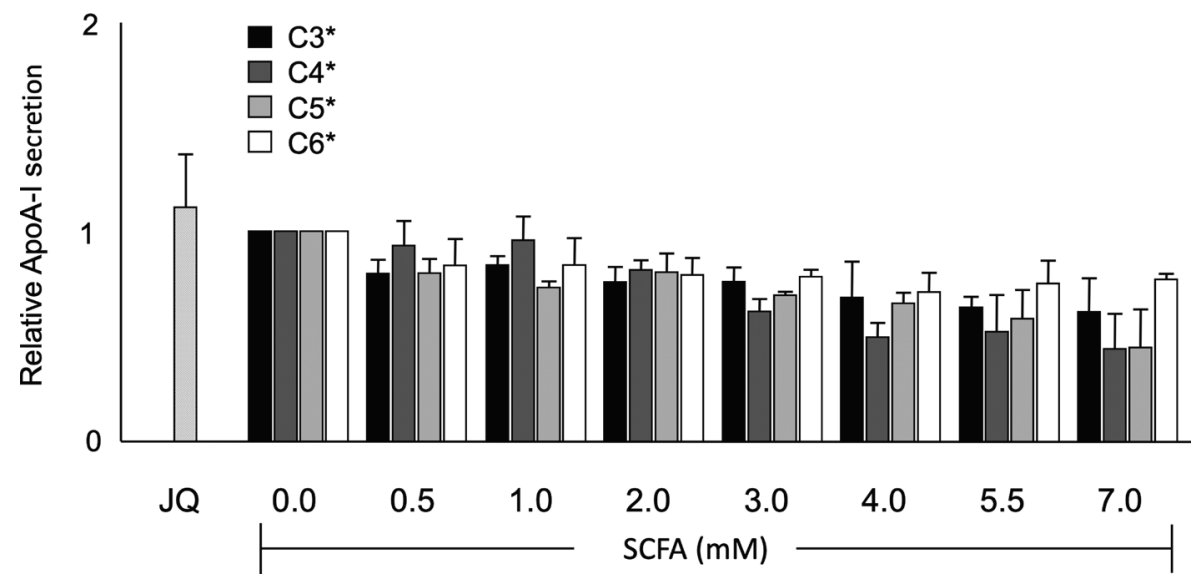

Figure 2.3. Relative ApoA-I protein secretion into the culture medium of HepG2 cells after treatment with different concentrations of SCFA. Increasing SCFA concentrations show significant regression coefficient deviated from zero $(\mathrm{p}<0.05)$ in ApoA-I protein secretion. JQ1 $(+)(3 \mu \mathrm{M})$ was used as a positive control for ApoA-I protein secretion. All results are presented as the mean, while error bars indicate standard deviations. Data were normalized against expression of the control condition, which was arbitrarily set at 1 . Changes are indicated with $\mathrm{a}^{*}$ when regression coefficient significantly at $(\mathrm{p}<0.05)$. ApoA-I, apolipoprotein-I; SCFA, short-chain fatty acids. 


\section{Effect of SCFA on KEAP1, CPT1 and PPARa mRNA expression}

To evaluate potential underlying mechanisms, changes in activities on the BET and PPAR $\alpha$ pathways were evaluated. In general, BET-inhibition is related to a decreased expression of the BET target gene KEAP1 and PPAR $\alpha$ transactivation via an increased expression of the PPARa target genes CPT1 and PPARa itself.

In general, SCFA treatment significantly and dose-dependently decreased KEAP1 gene expression ( $\mathrm{p}<0.001)$. In more detail, C3 decreased KEAP1 gene expression maximally by $50 \%$ at $3 \mathrm{mM}$, whereas $\mathrm{C} 4$ lowered KEAP1 expression even up to $80 \%$ at $7 \mathrm{mM}$. Moreover, KEAP 1 expression decreased by $60 \%$ and $50 \%$ after exposure to respectively $3 \mathrm{mM}$ C5 and C6. Interestingly, KEAP1 was downregulated while ApoA-I mRNA expressions was increased for all SCFA, suggesting that BET inhibition could be related to the observed increases in ApoA-I mRNA levels (Figure 2.4).

Moreover, CPT1 mRNA expression dose-dependently and significantly increased after SCFA treatment. More specifically, C3 significantly $(\mathrm{p}<0.001)$ increased CPT1 mRNA expression with a maximum of a 2.7 -fold at $7 \mathrm{mM}$. C4 was the strongest activator, i.e. a 3.6-fold CPT1 expression at $3 \mathrm{mM}$. In line with ApoA-I, this C4-induced elevation in CPT1 expression was followed by gradual reductions at higher C4 doses $(\mathrm{p}<0.001)$. C5 significantly $(\mathrm{p}<0.001)$ increased CPT1 gene expression to 1.9 -fold at $7 \mathrm{mM}$, whereas C6 was the weakest activator, i.e. only a slight but not significant 1.2 -fold increase at $3 \mathrm{mM}$ was observed (Figure 2.4).

In line with CPT1, all SCFA elevated PPARa gene expression, although effects of C4 fluctuated. Both C3 and C6 significantly increased PPARa expression to 4.0-fold and 4.4fold respectively $(\mathrm{p}<0.001)$. Like for ApoA-I and CPT1, C4 increased PPARa expression 1.9-fold with the most pronounced effect at $3 \mathrm{mM}$. At higher doses, a reduction of PPARa expression was observed. C5 significantly $(\mathrm{p}<0.01)$ increased PPAR $\alpha$ expressions to 1.8fold at $7 \mathrm{mM}$ (Figure 2.4).

To evaluate whether the effects of SCFA on CPT1 and PPAR $\alpha$ mRNA expressions could be explained via PPARa activation, we evaluated changes in PPAR $\alpha$ transcriptional activity with $\mathrm{C} 4$ as a model compound for all SCFA tested, in transfected HepG2 cells using a PPRE luciferase reporter system. In line with ApoA-I, CPT1 and PPARa mRNA expressions, C4 significantly increased PPAR $\alpha$ transactivation, followed by a gradual dose-dependent decrease resulting in an inhibition of $90 \%$ of basal PPARa transactivation at $5.5 \mathrm{mM}$ $(\mathrm{p}<0.001)$. Interestingly, JQ1(+) markedly inhibited PPAR $\alpha$ transactivation by 70\% (Figure 2.5). 
C3

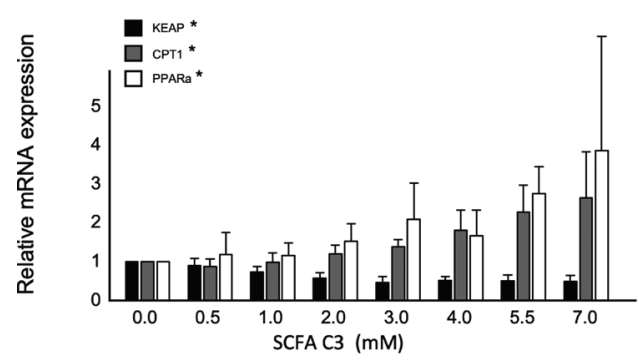

C5

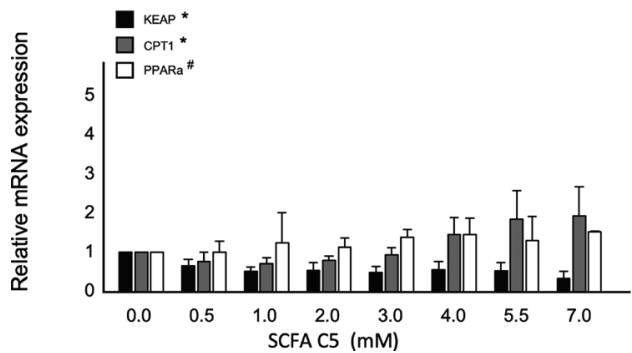

\section{C4}


Figure 2.4. Relative KEAP1, CPT1 and PPAR $\alpha$ mRNA expression in HepG2 cells treated with different concentrations of SCFA. C3 different concentrations show significant regression coefficient deviated from zero $(\mathrm{p}<0.001)$ in KEAP1, CPT1 and PPAR $\alpha$ mRNA expression. C4 different concentrations show significant regression coefficient deviated from zero $(\mathrm{p}<0.001)$ in KEAP1 and CPT1 and PPAR $\alpha$ mRNA expression. C5 different concentrations show significant regression coefficient deviated from zero $(p<0.001)$ in KEAP1 and CPT1 $(\mathrm{p}<0.01)$ in PPARa mRNA expression. C6 different concentrations show significant regression coefficient deviated from zero $(\mathrm{p}<0.001)$ in KEAP1 and PPAR $\alpha$ mRNA expression. Linear regression for SCFA dose-response effects was performed except C4, quadratic polynomial regression was performed for C4 dose-response effects. All results are presented as the mean, while error bars indicate standard deviations. Data were normalized against expression of the control condition, which was arbitrarily set at 1 . Changes are indicated with $\mathrm{a}^{*}$ when regression coefficient significantly at $(\mathrm{p}<0.001)$ or with a \# when regression coefficient significantly at $(\mathrm{p}<0.01)$. mRNA, messenger RNA; SCFA, short-chain fatty acids.

Overall, for all SCFA the patterns between CPT1 as well as PPARa and ApoA-I mRNA expressions and PPAR $\alpha$ transactivation were comparable suggesting that PPAR activation might relate to the observed elevation in ApoA-I mRNA levels.

\section{Discussion}

We here report a series of in vitro experiments in HepG2 cells supporting our hypothesis that antibiotics could have direct and indirect effects on hepatic ApoA-I transcription and secretion. First, we showed that amoxicillin lowered ApoA-I transcription and secretion by HepG 2 cells, whereas all SCFA tested, which are metabolites produced by microbiota, 


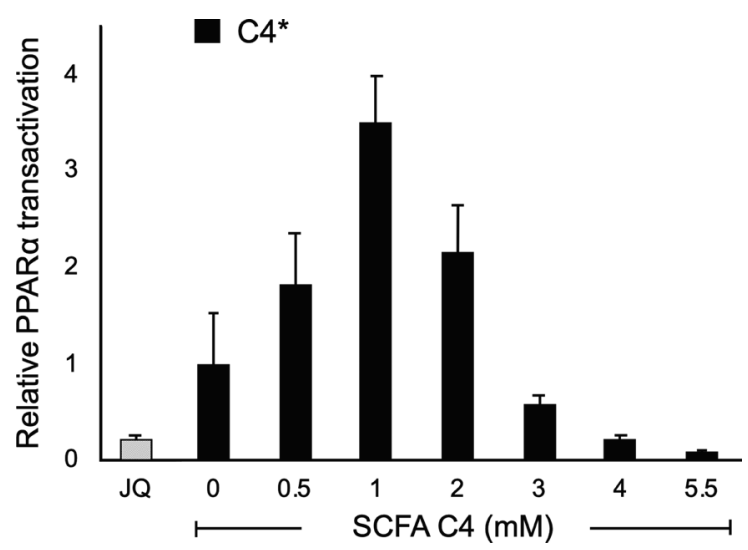

Figure 2.5. Relative PPARa transactivation in transfected HepG2 cells treated with different concentrations of $\mathrm{C} 4$. Increasing $\mathrm{C} 4$ concentrations show significant regression coefficient deviated from zero $(\mathrm{p}<0.001)$ in PPARa transactivation. All results are presented as the mean, while error bars indicate standard deviations. Data were normalized against expression of the control condition, which was arbitrarily set at 1 . Changes are indicated with a ${ }^{*}$ when regression coefficient significantly at $(\mathrm{p}<0.001)$.

clearly increased ApoA-I transcription, except for $\mathrm{C} 4$ at higher concentrations. This suggests that increasing the flux of SCFA from the portal vein towards the liver might translate into an increased hepatic ApoA-I transcription. Mechanistically, we showed that SCFAinduced BET inhibition as well as PPAR $\alpha$ activation suggesting that these two pathways are potentially involved.

Recently, we observed in healthy human volunteers that amoxicillin, a broad-spectrum antibiotic, significantly lowered serum HDL-C concentrations (1). Amoxicillin is a member of $\beta$-lactam antibiotics, which negatively affects bacterial growth by binding to a primary receptor, called the penicillin-binding protein ( $\mathrm{PBP})$, on the bacterial membrane $(21,22)$. As a consequence, bacteria lose their control over autolytic enzymes leading to apoptosis (23). As such, amoxicillin has a major impact on microbiota composition and given the enormous number of observations regarding the role of microbiota on human metabolism, antibiotics could theoretically affect numerous processes (24). Besides a direct effect of amoxicillin, our findings also suggest that changing microbiota composition which theoretically also changes SCFA production, impact hepatic ApoA-I expression. In other words, besides the direct gene regulating effect, it is well possible that the observed effects of amoxicillin treatment in humans on HDL-cholesterol concentrations are indirect, i.e. mediated by altering the composition of the microbiota which are responsible for the production of SCFA (2).

ApoA-I mRNA expression was stimulated by $\mathrm{C} 3, \mathrm{C} 4, \mathrm{C} 5$ and $\mathrm{C} 6$ treatments. At the same time, BET was inhibited and PPARa transactivated, suggesting that both BET inhibition 
as well as PPARa activation are involved in the effect of SCFA on ApoA-I expression. Interestingly, PPARa transactivation closely followed the same pattern, though not fully, which suggests that additional factors could be involved. These factors might include large families of co-activators and co-repressors molecules which together determine the net outcome of PPARa mediated effects (25). PPARa can be activated by recruitment of coactivators such as CBP and SRC1, but can be inhibited by binding of co-repressors such as SMRT and NCoR (26). On the other hand, increased ApoA-I expression with the BET inhibitor JQ1(+) was observed, even though JQ1(+) failed to induce PPARa transactivation. This indicates that ApoA-I expression could also increase due to other mechanisms besides PPARa transactivation, for instance via BET inhibition. This is also in agreement with our previous research in which we showed a clear role for BET inhibition, for example via exposure to JQ1(+), on ApoA-I expression (9). JQ1(+) binds to acetylated lysine binding sites within BET bromodomains, leading to BET proteins dissociation from chromatin, amongst others translating into elevated ApoA-I mRNA expression in HepG2 cells (27). Additionally, JQ1(+) also inhibited PPAR $\alpha$ transactivation as PPAR $\alpha$ and CTP1 expressions were both downregulated. This means that the net results of BET inhibitors on ApoA-I are actually very strong, since they also need to overcome the effect of a lower PPARa activation.

A puzzling question that remains is why after exposure to the SCFA ApoA-I concentrations in culture medium were in contrast to the elevated ApoA-I mRNA levels, significantly reduced. The lower secretion of ApoA-I into culture medium suggest that the higher level of ApoA-I mRNA is not translated, or ApoA-I protein is produced, but possibly remains trapped within the hepatocytes. Furthermore, it is also possible that ApoA-I was internalized and recycled back to the cell surface, which might be reason behind the reduced level of protein secreted (28). Moreover, the incubation period $48 \mathrm{~h}$ seems suitable for analyzing changes in ApoA-I mRNA expression, but for the changes in protein secretion this time frame could be relatively short. A study by Hahn and coworkers (29) has shown a significant effect on ApoA-I protein secretion only after three days of incubation. However, it should be mentioned that our positive control JQ1(+) did elevate ApoA-I protein secretion after $48 \mathrm{~h}$.

We observed some variation in SCFA ability to increase ApoA-I expression. This variation might be due to differences in binding strength of the SCFA to PPAR $a$ or BET. As mentioned before, several of co-activators and co-repressors molecules mediate PPAR $\alpha$ effects (25), SCFA (e.g. C4 at higher doses) may activate these co-repressor domains instead of activating the co-activator domains of PPARa, which can explain the inhibitory effects of $\mathrm{C} 4$ higher doses on PPARa transactivation and ApoA-I mRNA expression as well. 
Of all SCFA evaluated, $\mathrm{C} 4$ was at lower doses the most promising SCFA regarding elevating ApoA-I expression. Therefore, it might be interesting to evaluate the effects of C4 on ApoA-I production in future human intervention studies. Previous study have demonstrated that specific fibers such as resistant starch increased C4 formation by the microbiota (30). Biscuits rich in resistant starch type 3 improved glucose and lipid profiles in diabetic mice (31). Furthermore, a positive correlation between starch-rich food consumption and increased number of HDL particles was observed, this correlation resulted in lipid profile improvement (32).

In summary, we have shown that amoxicillin treatment has either direct effects on lowering ApoA-I transcription and secretion, or indirect effects via modified SCFA concentrations, since SCFA were found to stimulate hepatic ApoA-I expression. Effects of all four SCFA tested on ApoA-I mRNA expression showed a dose-dependent increase - except for C4 higher doses -. Finally, based on findings regarding KEAP1, CPT1 and PPARa gene expression as well as PPAR transactivation, it is tempting to suggest that both BET inhibition and PPAR $\alpha$ activation are potential mechanisms behind the observed SCFA induced effects on ApoA-I expression. A more detailed understanding of these mechanisms would add to new insights for approaches in elevating HDL levels to prevent and treat atherosclerosis and CVD.

\section{References}

1. Reijnders, D.; Goossens, Gijs H.; Hermes, Gerben D. A., et al. Effects of Gut Microbiota Manipulation by Antibiotics on Host Metabolism in Obese Humans: A Randomized DoubleBlind Placebo-Controlled Trial. Cell Metab. 2016, 24, 63-74.

2. De Smet. Plant stanol esters: Focus on intestinal lipoprotein metabolism. (Doctoral dissertation), Maastricht University, Maastricht, Netherlands 2014. Available from BOXPress (ISBN: 978-90-8891-909-1).

3. Khera, A. V.; Cuchel, M.; de la Llera-Moya, M.; Rodrigues, A., et al. Cholesterol efflux capacity, high-density lipoprotein function, and atherosclerosis. N Engl J Med. 2011, 364, 127-135.

4. Haas, M. J.; Reinacher, D.; Li, J. P.; Wong, N. C.; Mooradian, A. D. Regulation of apoA1 gene expression with acidosis: requirement for a transcriptional repressor. J. Mol. Endocrinol. 2001, 27, 43-57.

5. Du, Y.; Wang, L.; Si, S.; Yang, Y.; Hong, B. A novel compound 4010B-30 upregulates apolipoprotein A-I gene expression through activation of PPARgamma in HepG2 cells. Atherosclerosis. 2015, 239, 589-598.

6. Layden, B. T.; Angueira, A. R.; Brodsky, M.; Durai, V.; Lowe, W. L. Short chain fatty acids and their receptors: new metabolic targets. Transl Res. 2013, 161, 131-140.

7. Wong, J. M. W.; de Souza, R.; Kendall, C. W. C.; Emam, A.; Jenkins, D. J. A. Colonic Health: Fermentation and Short Chain Fatty Acids. J. Clin. Gastroenterol. 2006, 40, 235-243. 
8. Zhao, Y.; Liu, J.; Hao, W.; Zhu, H.; Liang, N.; He, Z.; Ma, K. Y.; Chen, Z.-Y. Structure-Specific Effects of Short-Chain Fatty Acids on Plasma Cholesterol Concentration in Male Syrian Hamsters. J. Agric. Food Chem. 2017, 65, 10984-10992.

9. Martin, G.; Duez, H.; Blanquart, C.; Berezowski, V.; Poulain, P.; Fruchart, J. C.; Najib-Fruchart, J.; Glineur, C.; Staels, B. Statin-induced inhibition of the Rho-signaling pathway activates PPARalpha and induces HDL apoA-I. J. Clin. Investig. 2001, 107, 1423-1432.

10. Van der Krieken, S. E.; Popeijus, H. E.; Mensink, R. P.; Plat, J. Link Between ER-Stress, PPAR-Alpha Activation, and BET Inhibition in Relation to Apolipoprotein A-I Transcription in HepG2 Cells. J. Cell. Biochem. 2017, 118, 2161-2167.

11. Jahagirdar, R.; Zhang, H.; Azhar, S.; Tobin, J.; Attwell, S.; Yu, R.; Wu, J. et al. A novel BET bromodomain inhibitor, RVX-208, shows reduction of atherosclerosis in hyperlipidemic ApoE deficient mice. Atherosclerosis. 2014, 236, 91-100.

12. Kempen, H. J.; Bellus, D.; Fedorov, O.; Nicklisch, S.; Filippakopoulos, P.; Picaud, S.; Knapp, S. Stimulation of Hepatic Apolipoprotein A-I Production by Novel Thieno-Triazolodiazepines: Roles of the Classical Benzodiazepine Receptor, PAF Receptor, and Bromodomain Binding. Lipid insights. 2013, 6, 47-54.

13. Hussong, M.; Börno, S. T.; Kerick, M.; Wunderlich, A.; Franz, A.; Sültmann, H.; Timmermann, B.; Lehrach, H.; Hirsch-Kauffmann, M.; Schweiger, M. R. The bromodomain protein BRD4 regulates the KEAP1/NRF2-dependent oxidative stress response. Cell Death Dis. 2014, 5, e1195.

14. Shi, J.; Vakoc, Christopher R. The Mechanisms behind the Therapeutic Activity of BET Bromodomain Inhibition. Mol. Cell 2014, 54, 728-736.

15. Berger, J.; Moller, D. E. The Mechanisms of Action of PPARs. Annu. Rev. Med. 2002, 53, 409-435.

16. Brown, J. D.; Plutzky, J. Peroxisome proliferator-activated receptors as transcriptional nodal points and therapeutic targets. Circulation. 2007, 115, 518-533.

17. Popeijus, H. E.; van Otterdijk, S. D.; van der Krieken, S. E.; Konings, M.; Serbonij, K.; Plat, J.; Mensink, R. P. Fatty acid chain length and saturation influences PPARa transcriptional activation and repression in HepG2 cells. Mol. Nutr. Food Res. 2014, 58, 2342-2349.

18. Tan, J.; McKenzie, C.; Potamitis, M.; Thorburn, A. N.; Mackay, C. R.; Macia, L.: Chapter Three - The Role of Short-Chain Fatty Acids in Health and Disease. In Adv. Immunol.; Alt, F. W., Ed.; Academic Press, 2014, 21, 91-119.

19. Vu-Dac, N.; Chopin-Delannoy, S.; Gervois, P.; Bonnelye, E., et al. The nuclear receptors peroxisome proliferator-activated receptor alpha and Rev-erbalpha mediate the species-specific regulation of apolipoprotein A-I expression by fibrates. J. Biol. Chem. 1998, 273, 25713-25720.

20. Arancibia, A.; Guttmann, J.; González, G.; González, C. Absorption and disposition kinetics of amoxicillin in normal human subjects. Antimicrob Agents Chemother. 1980, 17, 199-202.

21. Okamoto, T.; Yoshiyama, H.; Nakazawa, T.; Park, I. D.; Chang, M. W.; Yanai, H.; Okita, K.; Shirai, M. A change in PBP1 is involved in amoxicillin resistance of clinical isolates of Helicobacter pylori. J. Antimicrob. Chemother. 2002, 50, 849-856.

22. Guillemot, D.; Carbon, C.; Balkau, B.; et al. Low dosage and long treatment duration of $\beta$-lactam: Risk factors for carriage of penicillin-resistant streptococcus pneumoniae. JAMA 1998, 279, 365370 .

23. Tipper, D. J. Mode of action of $\beta$-lactam antibiotics. Pharmacol. Ther. 1985, 27, 1-35.

24. Ianiro, G.; Tilg, H.; Gasbarrini, A. Antibiotics as deep modulators of gut microbiota: between good and evil. Gut. 2016. 
25. Cronet, P.; Petersen, J. F. W.; Folmer, R.; Blomberg, N.; Sjöblom, K.; Karlsson, U.; Lindstedt, E.-L.; Bamberg, K. Structure of the PPAR $\alpha$ and $-\gamma$ Ligand Binding Domain in Complex with AZ 242; Ligand Selectivity and Agonist Activation in the PPAR Family. Structure. 2001, 9, 699-706.

26. Pourcet, B.; Pineda-Torra, I.; Derudas, B.; Staels, B.; Glineur, C. SUMOylation of human peroxisome proliferator-activated receptor alpha inhibits its trans-activity through the recruitment of the nuclear corepressor NCoR. J. Biol. Chem. 2010, 285, 5983-5992.

27. McLure, K. G.; Gesner, E. M.; Tsujikawa, L.; Kharenko, O. A.; Attwell, S.; Campeau, E.; Wasiak, S.; Stein, A.; White, A.; Fontano, E.; Suto, R. K.; Wong, N. C.; Wagner, G. S.; Hansen, H. C.; Young, P. R. RVX-208, an inducer of ApoA-I in humans, is a BET bromodomain antagonist. PLoS One 2013, 8, e83190.

28. Arciello, A.; De Marco, N.; Del Giudice, R.; Guglielmi, F.; Pucci, P.; Relini, A.; Monti, D. M.; Piccoli, R. Insights into the fate of the N-terminal amyloidogenic polypeptide of ApoA-I in cultured target cells. J Cell Mol Med. 2011, 15, 2652-2663.

29. Hahn, S. E.; Goldberg, D. M. Modulation of lipoprotein production in Hep G2 cells by fenofibrate and clofibrate. Biochem. Pharmacol. 1992, 43, 625-633.

30. Venkataraman, A.; Sieber, J. R.; Schmidt, A. W.; Waldron, C.; Theis, K. R.; Schmidt, T. M. Variable responses of human microbiomes to dietary supplementation with resistant starch. Microbiome. 2016, 4, 33 .

31. Nugraheni, M.; Hamidah, S.; Auliana, R.: A Potential of Coleus Tuberosus Crackers Rich in Resistant Starch Type 3 Improves Glucose and Lipid Profile of Alloxan -Induced Diabetic Mice. Curr Res Nutr Food Sci Jour. 2017, 5, 308-319.

32. Rideout, T. C.; Harding, S. V.; Raslawsky, A.; Rempel, C. B. Dietary Resistant Starch Supplementation Increases High-Density Lipoprotein Particle Number in Pigs Fed a Western Diet. J. Diet. Suppl. 2017, 14, 334-345. 


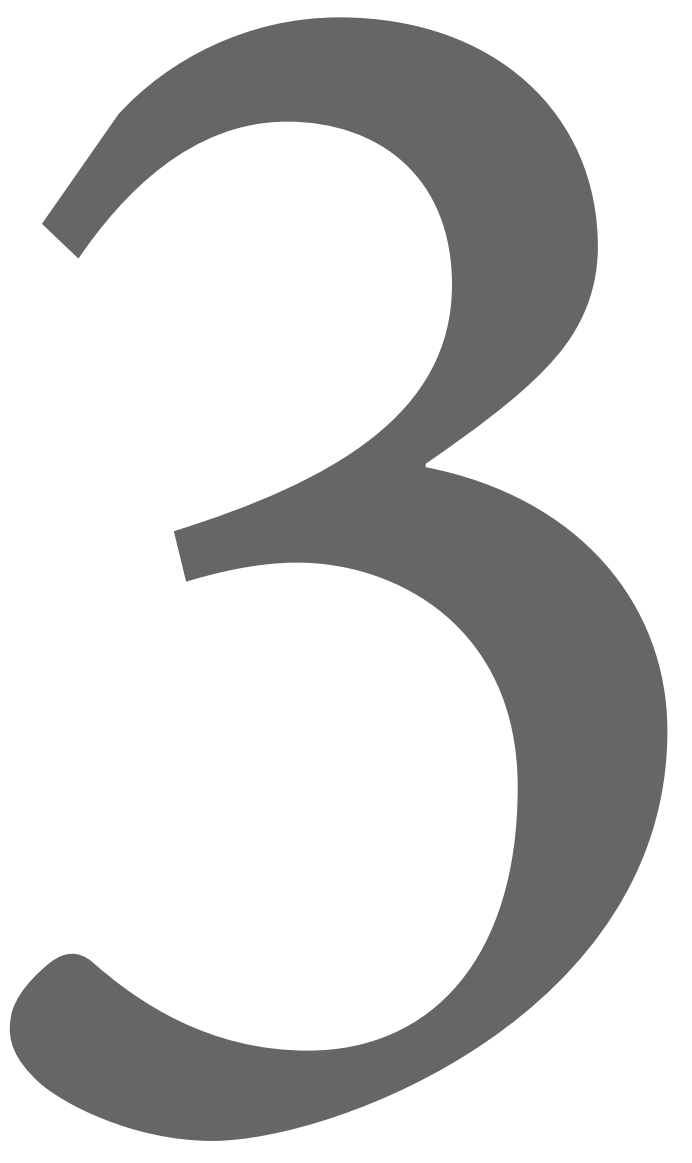




\section{Amoxicillin modulates ApoA-I transcription and secretion, predominantly via PPARa transactivation inhibition}

Jehad Z. Tayyeb | Herman E. Popeijus | Ronald P. Mensink | Maurice C.J.M. Konings | Kim H.R. Mulders | Jogchum Plat 


\section{Abstract}

In a recent human study, we observed that amoxicillin treatment decreased HDL-C concentration. We hypothesize that antibiotics lower the transcription and secretion of ApoA-I, the responsible protein for HDL production. HepG2 and Caco-2 cells were exposed to increasing dose of amoxicillin, penicillin, and streptomycin. Secreted ApoA-I protein and mRNA transcripts were analyzed using ELISA and qPCR, respectively. To unravel underlying mechanisms, KEAP1, CPT1, and CHOP mRNA expressions were determined as well as PPAR $\alpha$ transactivation. In HepG2 and Caco-2, amoxicillin decreased ApoA-I transcription and secretion. Effects on ApoA-I expression were clearly there for amoxicillin while no effects were observed for penicillin or streptomycin. KEAP1, CPT1, and CHOP mRNA expressions were reduced by amoxicillin treatments. Moreover, a significant correlation between ApoA-I and CPT1 mRNA expressions was found. Furthermore, amoxicillin lowered PPARa transactivation. All together, these data suggest that inhibited PPAR $\alpha$ transactivation is involved in the effects of amoxicillin on ApoA-I. In conclusion, the direct effect of amoxicillin in treated HepG2 and Caco-2 cells was a lower ApoA-I secretion and transcription. Based on evaluating alterations in KEAP1, CPT1, and CHOP mRNA expressions plus PPARa transactivation, we suggest that a reduced PPAR $a$ activation is a potential mechanism behind the observed amoxicillin effects on ApoA-I expression. 


\section{Introduction}

Frequently used antibiotics such as amoxicillin, penicillin, and streptomycin have been classified as essential drugs to treat many types of bacterial infections $(1,2)$. Besides treatment of bacterial infections, antibiotics also influence the quantity and composition of the natural microbiota, which may be involved in a wide variety of physiological processes (3). Over the last decades, evidence accumulated suggesting that the composition of the microbiota can be linked to cardiovascular disease (CVD) (4). Recent studies suggest that specific microbiota can influence atherosclerosis by altering inflammation processes and formation of microbial metabolites, and finally atherosclerotic plaques contain DNA of different bacterial species were found $(5,6)$. In addition, it has been found that gut microbiota is correlated with CVD biomarkers and lipid profiles including high-density lipoprotein-cholesterol (HDL-C) concentrations (7). The question is whether antibiotics might also affect CVD biomarkers, independent of effects on microbiota composition. In a recent placebo-controlled trial in healthy volunteers, we discovered that amoxicillin treatment significantly lowered HDL-C concentrations. Subjects were instructed to take 2 capsules of $250 \mathrm{mg}$ amoxicillin 3 times daily after each meal. One week after treatment, the $\mathrm{HDL}-\mathrm{C}$ concentration in the amoxicillin group was lower as compared to the concentration in the placebo group (8). However, the clinical relevance of this reduction as related to CVD is questionable since HDL functionality seems more important than HDL cholesterol concentrations (9). HDL functionality has been attributed to its main structural protein, ApolipoproteinA-I (ApoA-I), which amongst other effects mediates the process of cholesterol efflux. The cholesterol efflux capacity of the HDL fraction is representative of the removal of excess cholesterol from peripheral tissues by delivering it to the liver for excretion into bile (10). Since ApoA-I is the main functional protein in HDL, elevating the production of ApoA-I has become an interesting target for CVD prevention (11). In a recent overview, Smolders et al. have reviewed that ApoA-I mimetics as well as ApoA-I infusions (ApoA-I Milano, CSL-111, CSL-112, and CER001) and the BET inhibitor RVX208, which elevates ApoA-I production, have promising effects with respect to CVD (11). The synthesis of HDL starts with ApoA-I particles, and therefore, it is considered essential to form new, fresh, functional HDL particles. We hypothesized that the reduction in serum HDL cholesterol concentrations as observed after amoxicillin treatment is related to a reduced ApoA-I transcription. Therefore, we examined here the effects of different antibiotics, including amoxicillin, on ApoA-I transcription and secretion in hepatocytes and enterocytes. Moreover, to understand the underlying mechanism, we addressed the potential involvement of BET inhibition (12), PPARa transactivation $(13,14)$, or ER stress (15) on the antibiotic-induced changes in ApoA-I transcription. 


\section{Results}

Effects of JQ1(+) and Thap as controls on ApoA-I gene expression; ApoA-I protein secretion; and KEAP1, CPT1, and CHOP gene expressions in HepG2 and Caco-2 Cells

In agreement with previous results, JQ1(+) and Thap reacted as expected (16). To summarize, in HepG2 cells, the positive control JQ1(+) significantly increased ApoA-I gene expression whereas the negative control Thap decreased ApoA-I gene expression $(\mathrm{p}<$ 0.05). In line with ApoA-I gene expression, JQ1(+) significantly increased ApoA-I protein secretion whereas Thap significantly decreased ApoA-1 protein secretion $(\mathrm{p}<0.05)$. Moreover, as expected JQ1 $(+)$, a BET inhibitor, significantly $(\mathrm{p}<0.05)$ decreased KEAP1 gene expression and Thap increased KEAP1 gene expression. Furthermore, JQ1(+) significantly decreased CPT1 gene expression while Thap significantly increased CPT1 gene expression $(\mathrm{p}<0.05)$. Finally, JQ1 $(+)$ decreased CHOP gene expression while Thap significantly $(\mathrm{p}<0.05)$ increased CHOP gene expression (Figure 3.1).

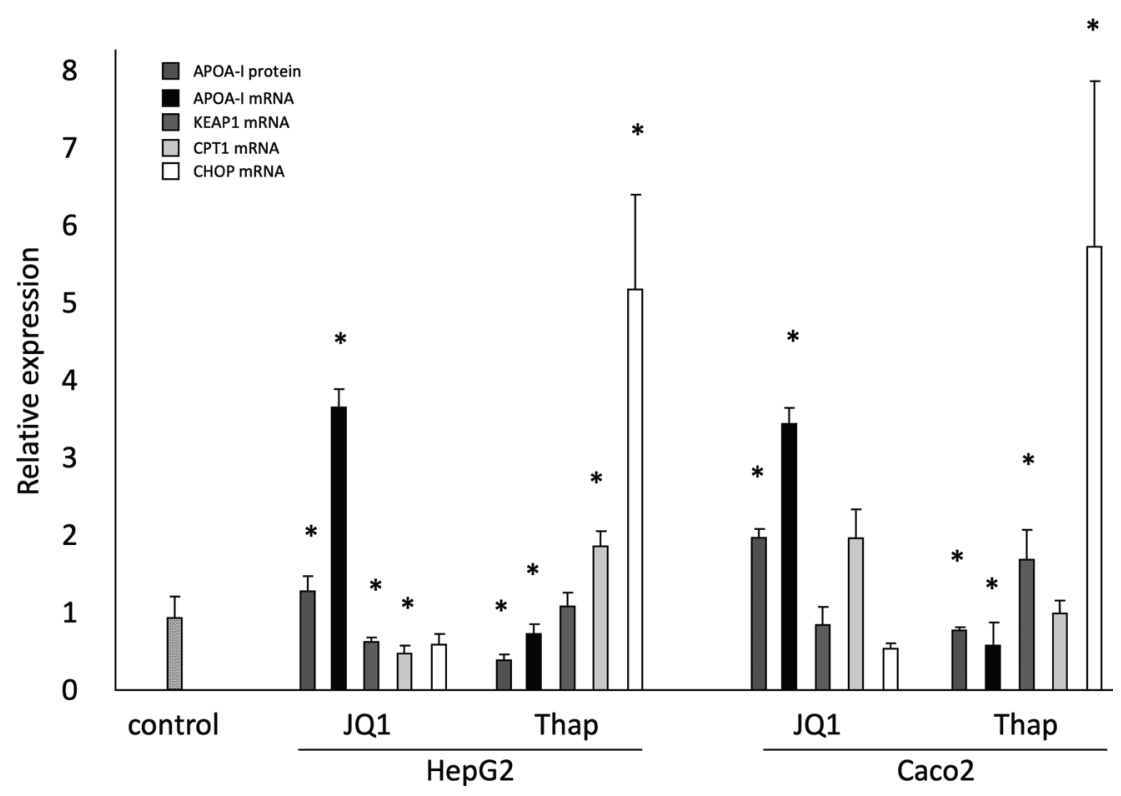

Figure 3.1. Relative ApolipoproteinA-I (ApoA-I) protein secretion and ApoA-I, KEAP1, CPT1, and CHOP mRNA expressions in HepG2 and Caco-2 cells treated with JQ1 $(+)(3 \mu \mathrm{M})$ or Thap $(0.01 \mu \mathrm{M})$ : Four biological (eight technical) replicates were performed for every condition. All results are presented as the mean, while error bars indicate standard deviations. Data were normalized against secretion or expression of the control condition (dimethyl sulfoxide (DMSO)), which was arbitrarily set at 1 . Changes are indicated with * when JQ1(+) or Thap are significantly different from control $(\mathrm{p}<0.05)$. DMSO, dimethyl sulfoxide; ApoA-I, apolipoprotein-I; mRNA, messenger RNA. 
In line with the effects observed in HepG2 cells, in Caco-2 cells JQ1(+) showed a significant increase in ApoA-I mRNA expression and ApoA-I protein secretion while Thap decreased ApoA-I gene expression and ApoA-I protein secretion $(\mathrm{p}<0.05)$. Moreover, JQ1(+) decreased KEAP1 gene expression, which did not reach statistical significance, whereas Thap significantly $(\mathrm{p}<0.05)$ increased KEAP1 expression in Caco-2 cells. Furthermore, both JQ1(+) and Thap did not affect CPT1 gene expression in Caco-2 cells. Finally, JQ1(+) decreased CHOP gene expression while Thap significantly $(\mathrm{p}<0.05)$ increased CHOP gene expression in Caco-2 cells (Figure 3.1).

\section{Effects of antibiotics on ApoA-I gene expression and protein secretion in HepG2 Cells}

Amoxicillin dose-dependently $(\mathrm{p}<0.05)$ lowered ApoA-I mRNA expression (Figure 3.2). Both penicillin and streptomycin as well as the combination of penicillin and streptomycin did not change ApoA-I mRNA expression. Moreover, amoxicillin also significantly $(\mathrm{p}<0.01)$, dose-dependently, decreased the amount of ApoA-I protein secreted into the culture medium (Figure 3.2). In contrast to ApoA-I mRNA, this dose-dependent reduction $(\mathrm{p}<0.01)$ was also found for streptomycin as well as for the combination of penicillin and streptomycin. Penicillin alone, however, did not have an effect on ApoA-I protein concentrations in culture medium. For amoxicillin, a significant correlation $(r=0.714$; $\mathrm{p}<0.05)$ was found between ApoA-I mRNA expression and ApoA-I protein secretion.

\section{Effects of antibiotics on KEAP1, CPT1, and CHOP gene expression and PPARa transactivation in HepG2cells}

Amoxicillin decreased KEAP1 gene expression significantly $(\mathrm{p}<0.01)$ and dosedependently. Penicillin did not affect KEAP1 gene expression, whereas streptomycin and the combination of penicillin and streptomycin significantly $(\mathrm{p}<0.05)$ decreased KEAP1 gene expression. Moreover, amoxicillin significantly $(\mathrm{p}<0.01)$ decreased CPT1 gene expression, whereas penicillin, streptomycin, and the combination of penicillin and streptomycin did not change CPT1 mRNA expression. Furthermore, amoxicillin significantly $(\mathrm{p}<0.05)$ decreased CHOP gene expression whereas penicillin, streptomycin, and the combination of penicillin and streptomycin did not change CHOP gene expression (Figure 3.2). In line with the lowering effect on CPT1 mRNA expression, amoxicillin also significantly $(\mathrm{p}<0.01)$ lowered PPAR $a$ transactivation. Penicillin $(\mathrm{p}<0.05)$ as well as the combination of penicillin and streptomycin $(\mathrm{p}<0.01)$ also significantly $(\mathrm{p}<0.05)$ decreased PPAR $\alpha$ transactivation, whereas streptomycin alone did not have an effect on PPAR $\alpha$ transactivation (Figure 3.3). Although amoxicillin lowered both ApoA-I and KEAP1 mRNA expressions, 
A) HepG2 cells

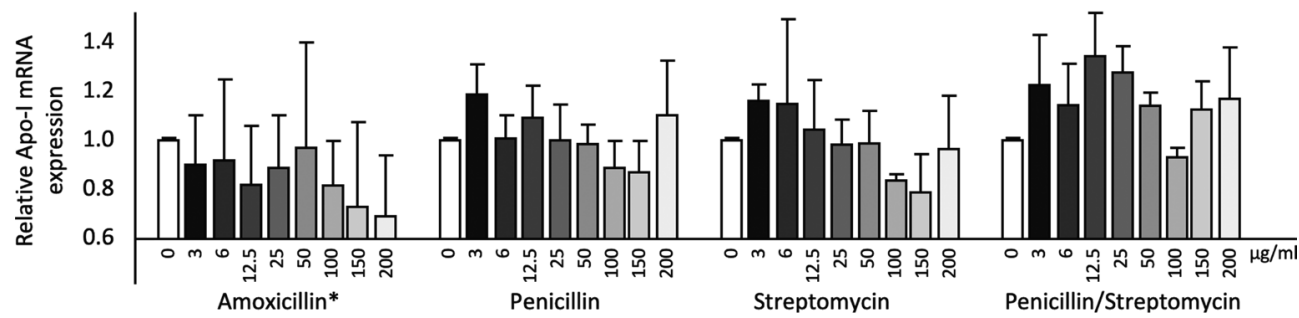

B)

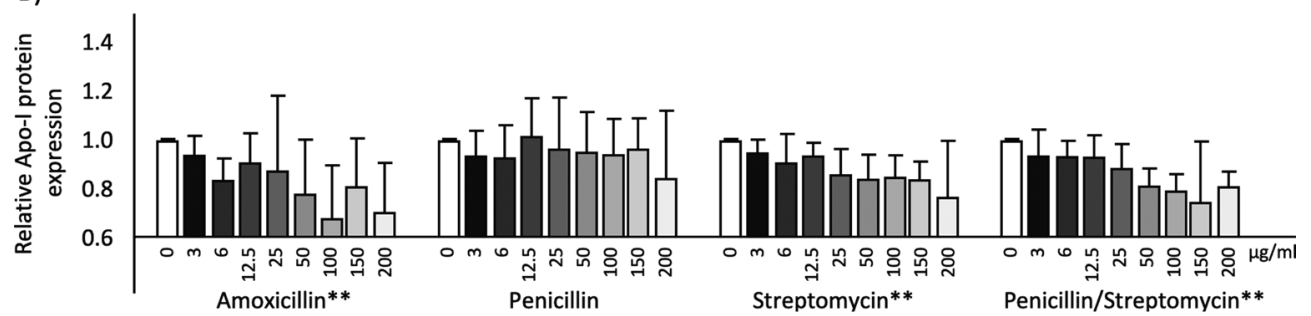

c)

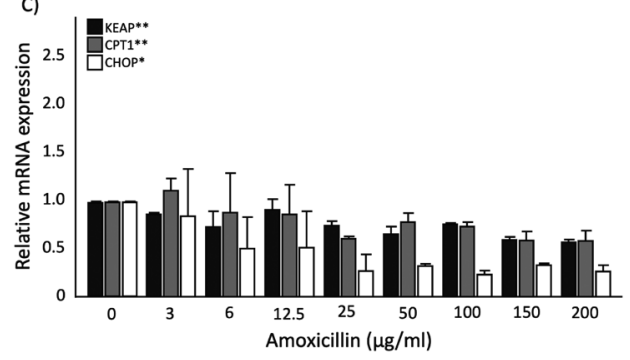

E)

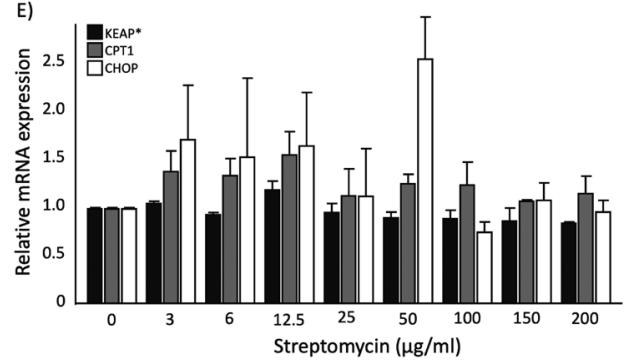

D)
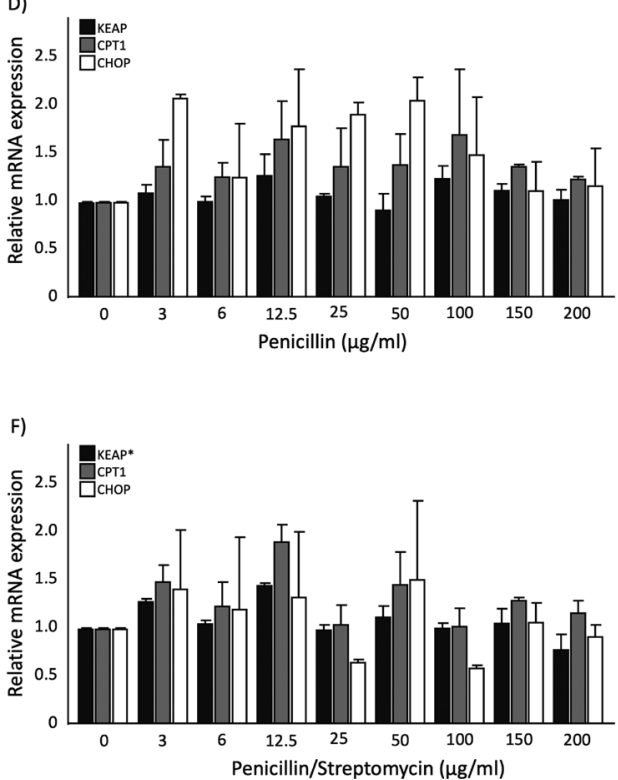

changes in the expression of these two genes were not significantly correlated $(r=0.510$; $\mathrm{p}=0.160)$. ApoA-I mRNA expression was significantly correlated $(\mathrm{r}=0.753 ; \mathrm{p}<0.05)$ with CPT1 mRNA expression, while both genes expressions were negatively correlated with the antibiotic treatment. Finally, a reduced CHOP mRNA expression was observed with amoxicillin treatment, but this effect was not significantly $(r=0.552 ; p=0.123)$ correlated with the reduction of ApoA-I mRNA expression. 
Figure 3.2. (A) Relative ApoA-I mRNA expression in HepG2 cells treated with different concentrations of antibiotics: Increasing amoxicillin concentrations showed a significant reduction in ApoA-I mRNA expression since the regression coefficient significantly different from zero $(\mathrm{p}<0.05)$. (B) Relative ApoA-I protein secretion into culture medium of HepG2 cells after treatment with different concentrations of antibiotics: Increasing amoxicillin, streptomycin, and the combination of penicillin and streptomycin concentrations showed a significant reduction in ApoA-I protein secretion since the regression coefficient significantly different from zero $(\mathrm{p}<0.01)$. (C - F) Relative KEAP1, CPT1, and CHOP mRNA expressions in HepG2 cells treated with different concentrations of antibiotics. (C) Increasing amoxicillin concentrations showed a significant reduction in KEAP1 and CPT1 mRNA expressions since the regression coefficient significantly different from zero $(p<0.01)$. Increasing amoxicillin concentrations showed a significant reduction in CHOP mRNA expression since the regression coefficient significantly different from zero $(\mathrm{p}<0.05)$. (D) Increasing penicillin concentrations did not show any significant effects in KEAP1, CPT1, and CHOP mRNA expressions. (E) Increasing streptomycin concentrations showed a significant reduction in KEAP1 mRNA expression since the regression coefficient significantly different from zero $(\mathrm{p}<0.05)$. (F) Increasing the combination of penicillin and streptomycin concentrations showed a significant reduction in KEAP1 mRNA expression since the regression coefficient significantly different from zero $(\mathrm{p}<0.05)$. Four biological (eight technical) replicates were performed for every single dose treatment. All results are presented as the mean, while error bars indicate standard deviations. Data were normalized against expression of the control condition, which was arbitrarily set at 1 . Changes are indicated with ${ }^{*}$ when the regression coefficient was significant $(\mathrm{p}<0.05)$ or with ${ }^{\star *}$ when the regression coefficient was significant $(\mathrm{p}<0.01)$. ApoA-I, apolipoprotein-I; mRNA, messenger RNA.

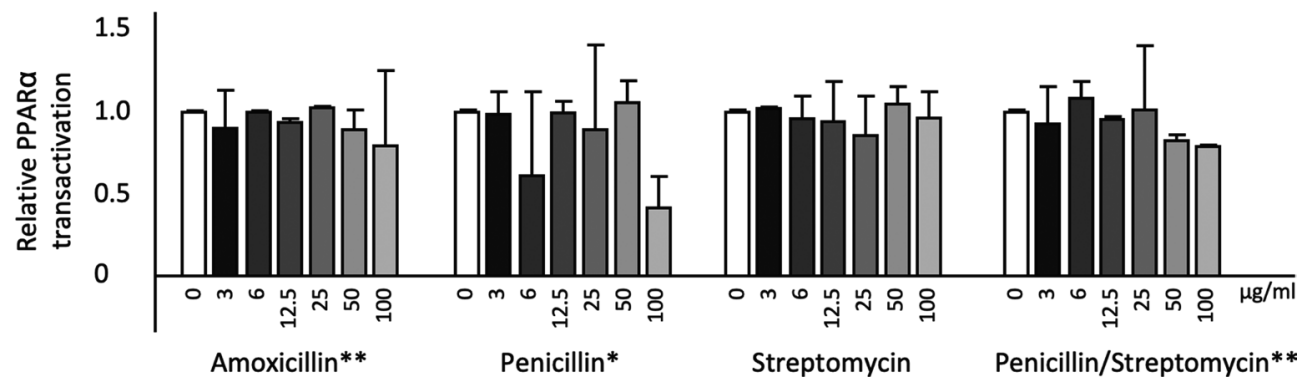

Figure 3.3. Relative PPARa transactivation in transfected HepG2 cells treated with different concentrations of antibiotics: Increasing amoxicillin and the combination of penicillin and streptomycin concentrations showed a significant reduction in PPAR $\alpha$ transactivation since the regression coefficient significantly different from zero $(p<0.01)$. Increasing penicillin concentrations showed a significant reduction in PPARa transactivation since the regression coefficient significantly different from zero $(\mathrm{p}<0.05)$. Four biological (eight technical) replicates were performed for every single dose treatment. All results are presented as the mean, while error bars indicate standard deviations. Data were normalized against expression of the control condition, which was arbitrarily set at 1 . Changes are indicated with $a^{*}$ when the regression coefficient was significant $(p<0.05)$ or with $\mathrm{a}^{* *}$ when the regression coefficient was significant $(\mathrm{p}<0.01)$.

\section{Effects of antibiotics on ApoA-I gene expression and protein secretion in Caco-2 Cells}

ApoA-1 mRNA expression was higher in differentiated Caco-2 cells as compared to HepG2 cells. As for HepG2 cells, amoxicillin significantly $(\mathrm{p}<0.01)$ and dose-dependently lowered ApoA-I mRNA expression in Caco-2 cells. Although both penicillin and streptomycin did not change ApoA-I mRNA expression, the combination of penicillin and streptomycin 
significantly $(\mathrm{p}<0.05)$ lowered dose-dependently ApoA-I gene expression (Figure 3.4). ApoA-1 protein secretion was also higher in differentiated Caco-2 cells as compared to HepG2 cells. In line with the change in ApoA-I mRNA expression, amoxicillin significantly $(\mathrm{p}<0.01)$ reduced dose-dependently the amount of ApoA-I protein secreted into the culture medium in Caco- 2 cells. This dose-dependent reduction $(\mathrm{p}<0.01)$ was also found for the other antibiotic treatments (Figure 3.4). Significant correlation was found between ApoA-I mRNA expression and ApoA-I protein secretion for the amoxicillin condition $(r=0.900$; $\mathrm{p}<0.05)$ as well as for the combination of penicillin and streptomycin $(\mathrm{r}=0.949 ; \mathrm{p}<0.05)$.

\section{Effects of antibiotics on KEAP1, CPT1, and CHOP gene expression in Caco-2 Cells}

As for HepG2 cells, amoxicillin decreased KEAP1 gene expression dose-dependently in Caco-2 cells $(\mathrm{p}<0.05)$. Although penicillin and streptomycin did not affect KEAP1 gene expression, the combination of penicillin and streptomycin also significantly lowered KEAP1 gene expression in a dose-dependent manner $(\mathrm{p}<0.01)$. Amoxicillin as well as the combination of penicillin and streptomycin significantly decreased CPT1 gene expression dose dependently $(\mathrm{p}<0.05)$. Moreover, penicillin significantly $(\mathrm{p}<0.05)$ increased CPT1 gene expression whereas streptomycin did not. Furthermore, in Caco-2 cells, CHOP mRNA expression was not changed after treatment with amoxicillin, penicillin, streptomycin, and the combination of penicillin and streptomycin (Figure 3.4). Although both amoxicillin and the combination of penicillin and streptomycin lowered ApoA-I and KEAP1 mRNA

Figure 3.4. (A) Relative ApoA-I mRNA expression in Caco-2 cells treated with different concentrations of antibiotics: Increasing amoxicillin concentrations showed a significant reduction in ApoA-I mRNA expression since the regression coefficient significantly different from zero $(p<0.01)$. Increasing the combination of penicillin and streptomycin concentrations showed a significant reduction in ApoA-I mRNA expression since the regression coefficient significantly different from zero $(\mathrm{p}<0.05)$. (B) Relative ApoA-I protein secretion into culture medium of Caco-2 cells after treatment with different concentrations of antibiotics. Increasing amoxicillin, penicillin, streptomycin, and the combination of penicillin and streptomycin concentrations showed a significant reduction in ApoA-I protein secretion since the regression coefficient significantly different from zero $(\mathrm{p}<0.01)$. (C - F) Relative KEAP1, CPT1, and CHOP mRNA expressions in Caco-2 cells treated with different concentrations of antibiotics: (C) Increasing amoxicillin concentrations showed a significant reduction in KEAP1 and CPT1 mRNA expressions since the regression coefficient significantly different from zero $(\mathrm{p}<0.05)$. (D) Increasing penicillin concentrations showed a significant increase in CPT1 mRNA expression since the regression coefficient significantly different from zero $(\mathrm{p}<0.05)$. (E) Increasing streptomycin concentrations did not show any significant effects in KEAP1, CPT1, and CHOP mRNA expressions. (F) Increasing the combination of penicillin and streptomycin concentrations showed a significant reduction in KEAP1 mRNA expression since the regression coefficient significantly different from zero $(\mathrm{p}<0.01)$. Increasing the combination of penicillin and streptomycin concentrations showed a significant reduction in CPT1 mRNA expression since the regression coefficient significantly different from zero $(\mathrm{p}<0.05)$. Four biological (eight technical) replicates were performed for every single dose treatment. All results are presented as the mean, while error bars indicate standard deviations. Data were normalized against expression of the control condition, which was arbitrarily set at 1 . Changes are indicated with ${ }^{\star}$ when the regression coefficient was significant $(\mathrm{p}<0.05)$ or with ${ }^{* *}$ when the regression coefficient was significant $(\mathrm{p}<0.01)$. ApoA-I, apolipoprotein-I; mRNA, messenger RNA. 


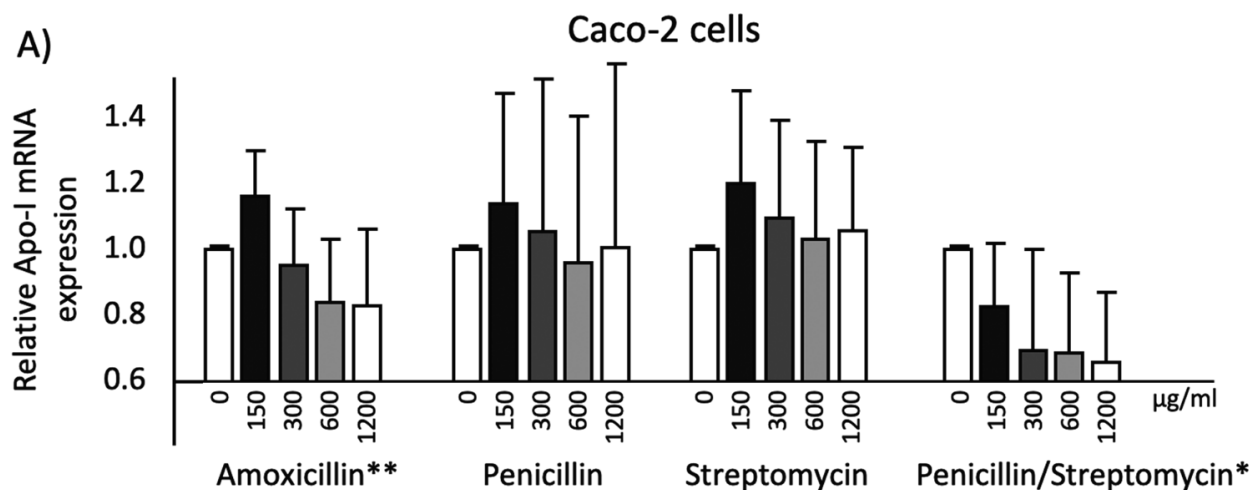

B)

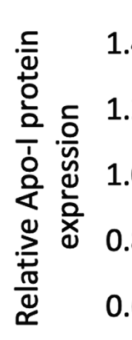

1.4

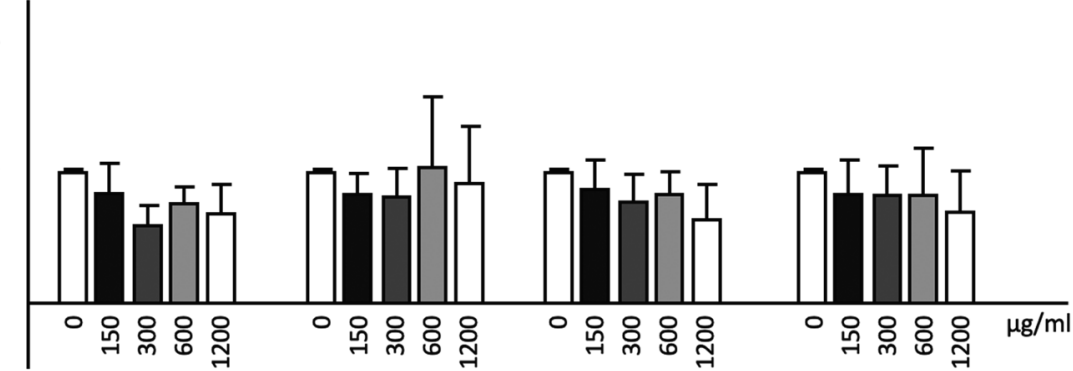

Amoxicillin** Penicillin** Streptomycin** Penicillin/Streptomycin**

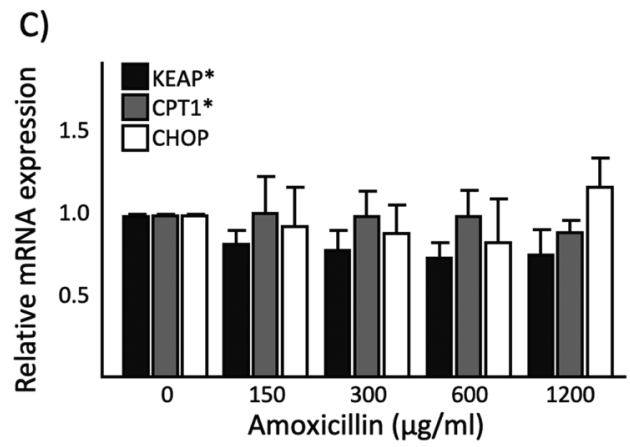

D)

E)

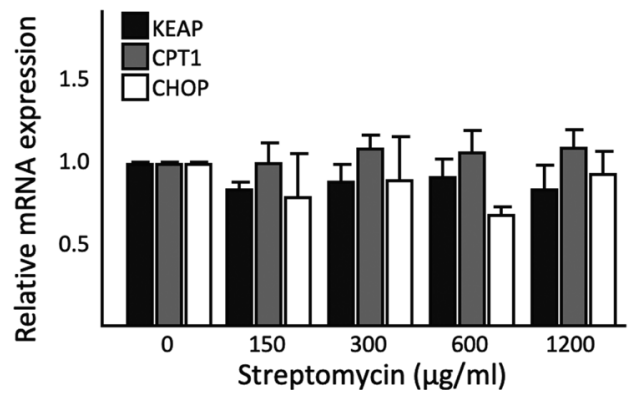



F)

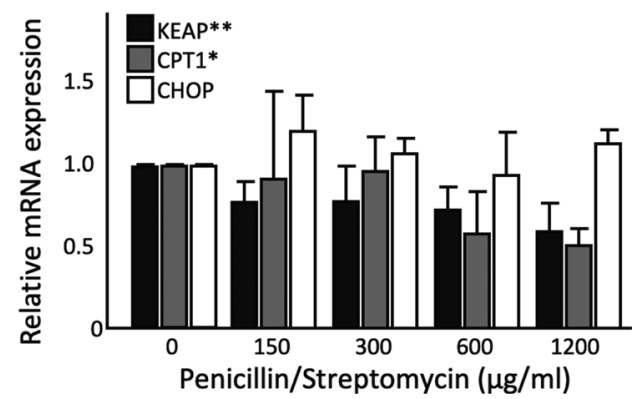


expressions, there was no significant correlation $(r=0.800 ; \mathrm{p}=0.104)$. However, significant correlations $(\mathrm{r}=0.975 ; \mathrm{p}<0.01$ and $\mathrm{r}=0.900 ; \mathrm{p}<0.05)$ were found between ApoA-I mRNA expression and CPT1 mRNA expression after exposure with amoxicillin and the combination of penicillin and streptomycin, respectively.

\section{Discussion}

Antibiotics are classified based on their chemical structures and modes of action. The generally used classes of antibiotics contain the aminoglycosides, beta-lactams, glycopeptides, macrolides, oxazolidinones, quinolones, sulphonamides, and tetracyclines (17). Here, we focused on amoxicillin and penicillin, which both belong to the beta-lactams, and on streptomycin, which belongs to the aminoglycosides class. Although these three antibiotics are considered as safe (18), there are some known "acceptable" side effects such as nausea, diarrhea, vomiting, allergic reactions, and hepatotoxicity (19-21). In addition, the effect of antibiotics on intestinal microbiota composition recently received major attention. These effects have been linked to diabetes development and CVD. Regarding CVD, we show here that one of these frequently used antibiotics, amoxicillin, had in vitro a direct negative effect on ApoA-I transcription and secretion by enterocytes and hepatocytes. Recently, we observed that amoxicillin treatment for 7 days significantly lowered serum HDL cholesterol in healthy subjects (8). Potential direct effects of antibiotics on human lipid and lipoprotein metabolism have, to the best of our knowledge, not been studied in humans before. Therefore, this is the first study to investigate the potential association between antibiotics treatment and ApoA-I gene expression and protein secretion. However, there are observations concerning a potential link between antibiotics and lipid metabolism in animal studies. Significant correlations were found between amoxicillin intake and changes in serum total cholesterol, triglycerides, and HDL and LDL concentrations in male rabbits (22). Moreover, Sato and coworkers (23) recently showed a significant reduction of intestinal ApoA-I secretion after four days of streptomycin and penicillin intake by Sprague-Dawley Rats.

Besides analyzing changes in ApoA-I transcription and secretion, we also explored the possible mechanisms behind this relation such as BET inhibition, PPAR $\alpha$ transactivation, or ER stress. BET protein inhibitors such as JQ1(+) increased ApoA-I expression $(24,25)$. BET inhibitors can regulate transcription of target genes such as KEAP1 $(12,26)$. PPARa is a nuclear receptor which binds to specific response elements (PPREs) within promoter regions of target genes such as CPT1 (14). PPARa activation is identified as targets to elevate ApoA-I transcription (16). ER stress markers like C/EBP homologous protein (CHOP) is activated by components that negatively influence the function of the endoplasmic 
reticulum (ER) (27). A previous study by Neam et al. reported that, in HepG2 cells, Thap resulted in an increase of ER-stress and a decrease of ApoA-I protein secretion (28).

Interestingly, by evaluating the mutual effects of the used agonists on the three pathways (PPARa activation, BET inhibition, and ER stress), some interesting issues concerning the relevance of the different pathways in ApoA-I transcription emerged. In line with our previous data, the BET inhibitor JQ1(+) significantly decreased KEAP1 gene expression; JQ1(+) also inhibited PPARa transactivation in HepG2 cells as CPT1 expressions were downregulated (16). This shows that the sum of effect by BET inhibitors on ApoA-I are quite strong, especially as they also need to counter its negative effect of a lower PPARa activation. JQ1(+) also decreased CHOP, suggesting that BET inhibition reduces ER stress. Interestingly, Thap not only elevated ER stress (higher CHOP expression) but also increased KEAP1 gene expression. This finding was expected since ER stress has opposite effects to BET inhibition in ApoA-I expression (16), meaning that ER stress is more likely to limit BET inhibition. Thap also increased PPARa transactivation in HepG2 cells as CPT1 expressions were increased, similar to JQ1(+); this means that the net results of ER stress on ApoA-I are potent, as they also need to counter the positive effect of a higher PPARa activation (Figure 3.5).

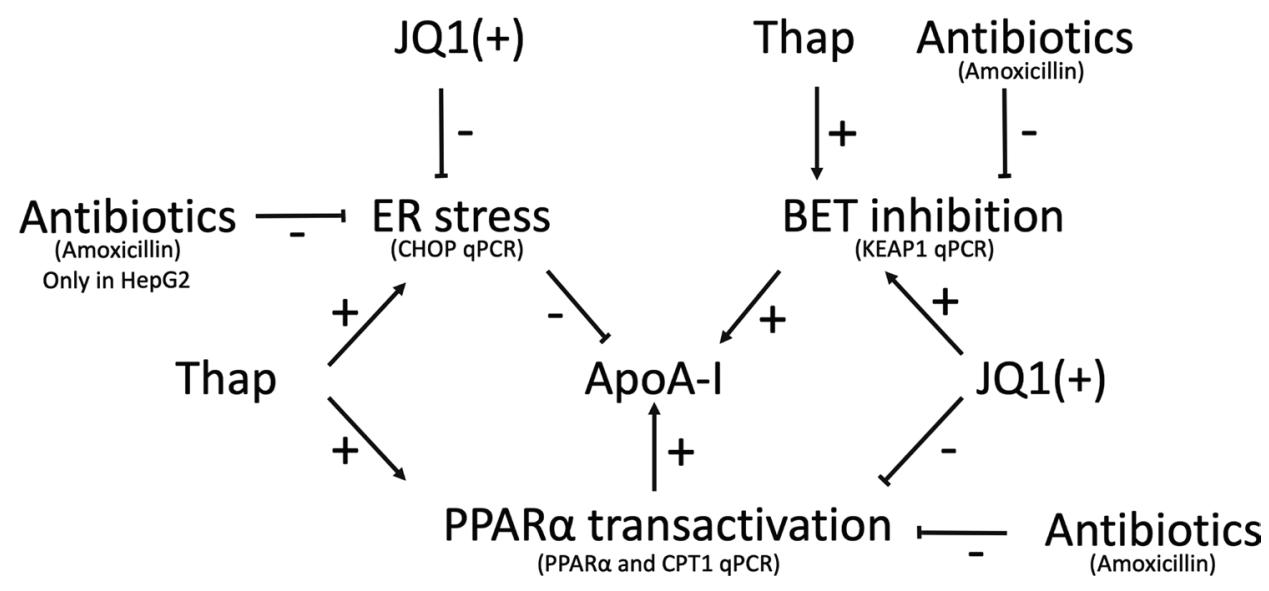

Figure 3.5. Schematic summary of the effects of antibiotics, JQ1(+) and Thap on pathways involved in ApoA-I mRNA expression: The lines represent effects responses (+ positive or - negative) in HepG2 and Caco- 2 cells. ApoA-I, apolipoprotein-I; mRNA, messenger RNA.

We observed differences in the ability of individual antibiotics to stimulate ApoA-I expression. As mentioned, the effects on ApoA-I expression were particularly evident for amoxicillin while no effects were observed for penicillin or streptomycin. The question now arises what differentiates amoxicillin from the other evaluated antibiotics. Amoxicillin, a $\beta$-lactam antibiotic, prevents bacterial growth through binding to the penicillin-binding 
protein $(\mathrm{PBP})$ that is present in the membrane of susceptible bacteria $(29,30)$. Moreover, amoxicillin induces protein damage; inhibits microsomal Ca2+ ATPase and G-6-Pase; and elevates membrane lipid peroxidation as result of increased production of reactive nitrogen species, reactive oxygen species, and free radicals. Finally, there is evidence that effects of amoxicillin might be the result of the attack of its $\beta$-lactam ring on the protein membrane and enzyme thiol groups (31). Although penicillin belongs to the same class as amoxicillin (beta-lactams), penicillin had no effects on the ApoA-I pathways studied. The possible reason is the presence of the $\mathrm{NH} 2$ functions in amoxicillin chemical structure. Amoxicillin has an additional amino acid chain $(-\mathrm{NH} 2)$ in the amide side chain and $(\mathrm{OH})$ in the para position. This makes amoxicillin acid resistant and more hydrophilic than penicillin (32). The hydrophilic force which regulates the molecular interactions between lipoproteins and enzymes (33) might be the reason behind the negative effects of amoxicillin on ApoA-I.

The experiments in HepG2 and Caco-2 cells presented here support our hypothesis that antibiotics elicit direct effects on ApoA-I secretion as well as transcription. We determined that amoxicillin lowered ApoA-I secretion and expression in both cell lines, and the question is how this effect can be explained mechanistically. As for ApoA-I expression, KEAP1, CPT1, and CHOP expressions were all reduced by amoxicillin treatments. Furthermore, we found a significant correlation between ApoA-I and CPT1 mRNA expressions after amoxicillin treatment. Moreover, amoxicillin had lowered PPARa transactivation in HepG2 cells. All together, these data support our previous finding that PPARa transactivation is involved in the effects of ApoA-I expression and secretion (16). Additionally, both BET inhibition and ER stress are most likely not involved in the relation between amoxicillin and ApoA-I expression.

There were some limitations of the in vitro system in this study, which could have some effects on the examined biomarkers outcome. For instance, cell lines are different from primary cells (in vivo) in some of their features and physiological responses (34). Additionally, the cell line responses to the antibiotics could be different compared to the patients' responses to same dose treatment; also, cells might have variations in their responses based on their passage numbers. Moreover, the cells were exposed to culture medium and serum, which can affect cells behavior, functions, and genetic information (35).

In summary, we have shown that amoxicillin treatment has direct effects by lowering ApoA-I secretion and transcription. Based on evaluating alterations in KEAP1, CPT1, and CHOP mRNA expression plus PPARa transactivation, it is tempting to suggest that a reduced PPAR $a$ transactivation is a potential mechanism behind the observed amoxicillininduced effects on hepatic and intestinal ApoA-I expression. 


\section{Material and methods}

\section{Materials}

Human hepatocellular liver carcinoma (HepG2) cells were obtained from Sten BraeschAndersen (Mabtech, Nacka Strand, Sweden). Human epithelial colorectal adenocarcinoma (Caco-2) cells were obtained from ATCC (Molsheim, France). Flasks and plates for cell culture were derived from Corning (Corning, NY, USA). Minimum Essential Medium (MEM), Dulbecco's Modified Eagle Medium (DMEM), sodium pyruvate, and nonessential amino acids (NEAA) used were purchased from Thermo Fisher Scientific (Bleiswijk, The Netherlands). Fetal bovine serum (FBS) was derived from PAA (Toronto, Canada). Amoxicillin, penicillin, streptomycin, DMSO, Tri-reagent, and Thapsigargin (Thap; an endoplasmic reticulum (ER) stress inducer) were obtained from Sigma (Uithoorn, The Netherlands). The BET inhibitor JQ1(+) was obtained from Bio-techne-R\&D (Minneapolis, MN, USA).

\section{Cell culture and antibiotics treatment}

HepG2 and Caco-2 cells were cultured under a humidified atmosphere with 5\% carbon dioxide $\left(\mathrm{CO}_{2}\right)$ in antibiotic free medium (MEM for HepG2 and DMEM for Caco-2) containing $1 \%$ sodium pyruvate, $10 \%$ heat inactivated FBS, and $1 \%$ NEAA at $37^{\circ} \mathrm{C}$. For experiments, cells were seeded at a density of 200,000 cells per well in a 24-well plate setup. HepG2 cells were grown to confluence for $48 \mathrm{~h}$; when cells reached a density of $80-90 \%$, they were exposed to the different antibiotics or JQ1(+) or Thap. Caco-2 cells were grown for 21 days to allow them to differentiate towards a small intestinal phenotype (36) before exposure to the same compounds as mentioned for the HepG2 cells. We used amoxicillin and penicillin $G$ (benzyl penicillin), both $\beta$-lactam antibiotics, and streptomycin, an aminoglycoside antibiotic (37-39). In addition, the cells were exposed to the combination of streptomycin and penicillin, which is often used prophylactically in cell cultures. In our previous human study where the reduction in serum HDL cholesterol concentrations was observed (8), capsules of $500 \mathrm{mg}$ amoxicillin were taken three times daily. If $500 \mathrm{mg}$ is dissolved in an assumed maximal volume of $3 \mathrm{~L}$ of stomach content (40), the cells of the small intestine will be exposed to a minimal concentration of about $167 \mu \mathrm{g} / \mathrm{mL}$ amoxicillin. Moreover, antibiotic local concentrations in the gastrointestinal tract might differ due to the presence of food or variable fluid consumption. Therefore, it was decided in this study to test the effects of the antibiotics on differentiated Caco- 2 cells in concentrations of 150, 300,600 , and $1200 \mu \mathrm{g} / \mathrm{mL}$. On the other hand, following oral administration of $500 \mathrm{mg}$ of amoxicillin, serum peak levels were between 6.0 to $15.3 \mu \mathrm{g} / \mathrm{mL}$, and after intravenous administration of $500 \mathrm{mg}$ of amoxicillin, serum peak levels were found to be between 52.1 
to $30.1 \mu \mathrm{g} / \mathrm{mL}$ (41). After oral administration of $500 \mathrm{mg}$ penicillin, blood serum levels were $3.8 \mu \mathrm{g} / \mathrm{mL}$ and intravenous administration resulted in serum levels between $13.9 \mu \mathrm{g} / \mathrm{mL}$ and $17 \mu \mathrm{g} / \mathrm{mL}$ (42). Intramuscular injection of $1 \mathrm{~g}$ of streptomycin showed a peak serum level of 25 to $50 \mu \mathrm{g} / \mathrm{mL}$ (43). Therefore, the effects of the selected antibiotics on HepG2 cells were initially tested at concentrations of $3,6,12.5,25,50,100,150$, and $200 \mu \mathrm{g} / \mathrm{mL}$. Both HepG2 as well as Caco-2 cells were exposed for $48 \mathrm{~h}$ in culture medium without added FBS enriched to the abovementioned concentration range antibiotics. In all experiments, JQ1(+) $(3 \mu \mathrm{M})$ and Thap $(0.01 \mu \mathrm{M})$ were used in separate wells as positive and negative controls for ApoA-I production, respectively (16). All antibiotics were dissolved in water, and their effects were expressed relative to a water control. JQ1(+) and Thap were both dissolved in dimethyl sulfoxide (DMSO) and their effects were therefore expressed relative to a DMSO control. Cell culture medium was collected after $48 \mathrm{~h}$ for analysis of ApoA-I protein concentrations. The cells were harvested to determine mRNA expression levels of ApoA-I, KEAP1, CPT1, CHOP and cyclophilin A. All samples, culture medium and lysed cells, were snap frozen in liquid nitrogen and stored at $-80^{\circ} \mathrm{C}$ prior to further analysis.

\section{ApoA-I protein concentration in cell culture medium}

ApoA-I protein concentrations in culture medium of both HepG2 and Caco-2 cells were measured by a direct enzyme-linked sandwich immunoassay (ELISA) obtained from Mabtech (Nacka Strand, Sweden) following the manufacturer's instructions, with small adaptations, e.g., blocker BSA 10\% (Thermo Fisher Scientific, Bleiswijk, Netherlands) was added to the block buffer (final concentration 1\%) and the dilution buffer (final concentration $0.1 \%$ ).

\section{mRNA expression quantification}

Total RNA was isolated using Tri-reagent to evaluate mRNA expression levels of ApoA-I, KEAP1, CPT1, and CHOP according to the manufacturer's instructions. The isolated RNA was further purified using the RNeasy mini kit (Qiagen, Hilden, Germany). Next, 350 ng of total RNA was reverse transcribed for cDNA synthesis, using RNAse inhibitor, dNTPs, random hexamers, MMLV reverse trans, DTT, and $5 \times$ FS buffer (Thermo Fisher Scientific, Bleiswijk, Netherlands). This cDNA served as a template for real time quantitative PCR using TaqMan Gene Expression Assays, ApoA-I (Hs 00163641), KEAP1 (Hs 00202227), CPT1 (Hs 00912671), and CHOP (Hs 00358796). Expression of the housekeeping cyclophilin A (Hs 99999904) was used as a control. Gene expression levels were presented as relative values based on the $\mathrm{Ct}$ values, normalized for the cyclophilin $\mathrm{A}$, and compared to their respective control conditions. 


\section{Luciferase assay}

PPAR $\alpha$ transcriptional activity was analyzed by transfection of HepG2 cells using X-treme gene 9 DNA transfection reagent (Sigma, Uithoorn, Netherlands) with the following plasmids: pcDNA3.1, pcDNA3.1_PPARa, pGL3, and pGL3_PPRE as previously described (44). Following transfection and 48-h antibiotics treatment, cells were harvested by lysis in $1 \times$ luciferase lysis buffer (Promega, Madison, USA) and luciferase activity, reflecting PPAR $\alpha$ transactivation, and was determined by a GloMax ${ }^{\circledR} 96$ Microplate luminometer, following the manufacturer's instructions (Promega, Madison, WI, USA). This transfection assay was only performed in HepG2 cells, since Caco- 2 cells need 21 days to be fully differentiated, as cells only remain transfected for 7 days maximally and the differentiated cells are typically resistant to transient transfection.

\section{Statistical analysis}

All independent experiments contained duplicate samples; furthermore, each independent experiment was repeated at least twice. Four biological (eight technical) replicates were performed for every single dose treatment. With regression analysis, the dose-response relationship between the antibiotic and the gene of interest was examined using a regression coefficient. Spearman correlations between ApoA-I protein secretion and ApoA-I mRNA expression or the mRNA expression of KEAP1, CPT1, and CHOP were calculated. The regression coefficient and spearman correlation coefficient were considered to be statistically significant at $\mathrm{p}<0.05$. All statistical analyses were performed using SPSS v.25 (IBM Corp., Armonk, NY, USA).

\section{References}

1. WHO. 19th WHO Model List of Essential Medicine. 2015. Available online: https://www.who. int/medicines/publications/essentialmedicines/EML2015_8-May-15.pdf (accessed on 8 October 2019).

2. Ventola, C.L. The antibiotic resistance crisis: Part 1: Causes and threats. P T. 2015, 40, 277-283.

3. Ianiro, G.; Tilg, H.; Gasbarrini, A. Antibiotics as deep modulators of gut microbiota: Between good and evil. Gut. 2016, 65, 1906-1915.

4. Lippi, G.; Danese, E.; Mattiuzzi, C.; Favaloro, E.J. The Intriguing link between the Intestinal microbiota and cardiovascular disease. Semin. Thromb. Hemost. 2017, 43, 609-613.

5. Battson, M.L.; Lee, D.M.; Weir, T.L.; Gentile, C.L. The gut microbiota as a novel regulator of cardiovascular function and disease. J. Nutr. Biochem. 2018, 56, 1-15.

6. Ma, J.; Li, H. The role of gut microbiota in atherosclerosis and hypertension. Front. Pharmacol. 2018, 9, 1082. 
7. Canyelles, M.; Tondo, M.; Cedó, L.; Farràs, M.; Escolà-Gil, J.C.; Blanco-Vaca, F. Trimethylamine N-Oxide: A link among diet, gut microbiota, gene regulation of liver and intestine cholesterol homeostasis and HDL function. Int. J. Mol. Sci. 2018, 19, 3228.

8. De Smet, E. Plant Stanol Esters: Focus on Intestinal Lipoprotein Metabolism. Ph.D. Thesis, Maastricht University, Maastricht, The Netherlands, 2014. Available from BOXPress, ISBN 97890-8891-909-1.

9. Khera, A.V.; Cuchel, M.; De La Llera-Moya, M.; Rodrigues, A.; Burke, M.F.; Jafri, K.; French, B.C.; Phillips, J.A.; Mucksavage, M.L.; Wilensky, R.L. Cholesterol efflux capacity, high-density lipoprotein function, and atherosclerosis. N. Engl. J. Med. 2011, 364, 127-135.

10. Du, Y.; Wang, L.; Si, S.; Yang, Y.; Hong, B. A novel compound 4010B-30 upregulates apolipoprotein A-I gene expression through activation of PPARgamma in HepG2 cells. Atherosclerosis. 2015, 239, 589-598.

11. Smolders, L.; Plat, J.; Mensink, R.P. Dietary strategies and novel pharmaceutical approaches targeting serum ApoA-I metabolism. J. Nutr. Metab. 2017, 2017, 5415921.

12. Shi, J.; Vakoc, C.R. The mechanisms behind the therapeutic activity of BET bromodomain inhibition. Mol. Cell. 2014, 54, 728-736.

13. Martin, G.; Duez, H.; Blanquart, C.; Berezowski, V.; Poulain, P.; Fruchart, J.C.; Najib-Fruchart, J.; Glineur, C.; Staels, B. Statin-induced inhibition of the Rho-signaling pathway activates PPARalpha and induces HDL apoA-I. J. Clin. Investig. 2001, 107, 1423-1432.

14. Berger, J.; Moller, D.E. The mechanisms of action of PPARs. Annu. Rev. Med. 2002, 53, 409-435.

15. Liu, M.Q.; Chen, Z.; Chen, L.X. Endoplasmic reticulum stress: A novel mechanism and therapeutic target for cardiovascular diseases. Acta Pharmacol. Sin. 2016, 37, 425-443.

16. Van der Krieken, S.E.; Popeijus, H.E.; Mensink, R.P.; Plat, J. Link between ER-stress, PPAR-alpha activation, and BET inhibition in relation to apolipoprotein A-I transcription in HepG2 cells. J. Cell. Biochem. 2017, 118, 2161-2167.

17. Etebu, E.; Arikekpar, I. Antibiotics: Classification and mechanisms of action with emphasis on molecular perspectives. Int. J. Appl. Microbiol. Biotechnol. Res. 2016, 4, 90-101.

18. Mandell, L.A.; Ball, P.; Tillotson, G. Antimicrobial safety and tolerability: Differences and dilemmas. Clin. Infect. Dis. 2001, 32, S72-S79.

19. Salvo, F.; De Sarro, A.; Caputi, A.P.; Polimeni, G. Amoxicillin and amoxicillin plus clavulanate: A safety review. Expert Opin. Drug Saf. 2009, 8, 111-118.

20. Bhattacharya, S. The facts about penicillin allergy. J. Adv. Pharm. Technol. Res. 2010, 1, 11-17.

21. Crofton, J. The MRC randomized trial of streptomycin and its legacy: A view from the clinical front line. J. R. Soc. Med. 2006, 99, 531-534.

22. Al-Jowari, S. Comparative investigation of amoxicillin and cephalexin administration on some biochemical parameters in male rabbits. IJSR 2018, 7, 1481-1484.

23. Sato, H.; Zhang, L.S.; Martinez, K.; Chang, E.B.; Yang, Q.; Wang, F.; Howles, P.N.; Hokari, R.; Miura, S.; Tso, P. Antibiotics suppress activation of intestinal mucosal mast cells and reduce dietary lipid absorption in Sprague-Dawley rats. Gastroenterology. 2016, 5, 923-932.

24. Jahagirdar, R.; Zhang, H.; Azhar, S.; Tobin, J.; Attwell, S.; Yu, R.; Wu, J.; McLure, K.G.; Hansen, H.C.; Wagner, G.S. A novel BET bromodomain inhibitor, RVX-208, shows reduction of atherosclerosis in hyperlipidemic ApoE deficient mice. Atherosclerosis. 2014, 236, 91-100. 
25. Kempen, H.J.; Bellus, D.; Fedorov, O.; Nicklisch, S.; Filippakopoulos, P.; Picaud, S.; Knapp, S. Stimulation of hepatic apolipoprotein A-I production by novel thieno-triazolodiazepines: Roles of the classical benzodiazepine receptor, PAF receptor, and bromodomain binding. Lipid Insights. 2013, 6,47-54.

26. Hussong, M.; Börno, S.; Kerick, M.; Wunderlich, A.; Franz, A.; Sültmann, H.; Timmermann, B.; Lehrach, H.; Hirsch-Kauffmann, M.; Schweiger, M. The bromodomain protein BRD4 regulates the KEAP1/NRF2-dependent oxidative stress response. Cell Death Dis. 2014, 5, e1195.

27. Wang, X.Z.; Lawson, B.; Brewer, J.W.; Zinszner, H.; Sanjay, A.; Mi, L.J.; Boorstein, R.; Kreibich, G.; Hendershot, L.M.; Ron, D. Signals from the stressed endoplasmic reticulum induce C/EBPhomologous protein (CHOP/GADD153). Mol. Cell. Biol. 1996, 16, 4273-4280.

28. Naem, E.; Haas, M.J.; Wong, N.C.; Mooradian, A.D. Endoplasmic reticulum stress in HepG2 cells inhibits apolipoprotein A-I secretion. Life Sci. 2013, 92, 72-80.

29. Guillemot, D.; Carbon, C.; Balkau, B.; Geslin, P.; Lecoeur, H.; Vauzelle-Kervroëdan, F.; Bouvenot, G.; Eschwége, E. Low dosage and long treatment duration of $\beta$-lactam: Risk factors for carriage of penicillin-resistant streptococcus pneumoniae. JAMA 1998, 279, 365-370.

30. Okamoto, T.; Yoshiyama, H.; Nakazawa, T.; Park, I.D.; Chang, M.W.; Yanai, H.; Okita, K.; Shirai, M. A change in PBP1 is involved in amoxicillin resistance of clinical isolates of Helicobacter pylori. $J$. Antimicrob. Chemother. 2002, 50, 849-856.

31. Adesanoye, O.; Ifezue, A.; Farombi, E. Influence of chloramphenicol and amoxicillin on rat liver microsomal enzymes and lipid peroxidation. Afr. J. Biomed. Res. 2014, 17, 135-142.

32. Neal, M.J. Medical Pharmacology at a Glance, 7th ed.; Wiley: Hoboken, NJ, USA, 2015; pp. 82-83.

33. Amigó, N.; Mallol, R.; Heras, M.; Martínez-Hervás, S.; Blanco-Vaca, F.; Escolà-Gil, J.C.; Plana, N.; Yanes, Ó.; Masana, L.; Correig, X. Lipoprotein hydrophobic core lipids are partially extruded to surface in smaller HDL: "Herniated" HDL, a common feature in diabetes. Sci. Rep. 2016, 6, 19249.

34. Pan, C.; Kumar, C.; Bohl, S.; Klingmueller, U.; Mann, M. Comparative proteomic phenotyping of cell lines and primary cells to assess preservation of cell type-specific functions. Mol. Cell. Proteom. 2009, 8, 443-450.

35. Savoji, H.; Mohammadi, M.H.; Rafatian, N.; Toroghi, M.K.; Wang, E.Y.; Zhao, Y.; Korolj, A.; Ahadian, S.; Radisic, M. Cardiovascular disease models: A game changing paradigm in drug discovery and screening. Biomaterials. 2019, 198, 3-26.

36. Van Breemen, R.B.; Li, Y. Caco-2 cell permeability assays to measure drug absorption. Expert Opin. Drug Metab. 2005, 1, 175-185.

37. DrugBank. Amoxicillin. 2005. Available online: https://www.drugbank.ca/drugs/DB01060 (accessed on 8 October 2019).

38. DrugBank. Benzylpenicillin. 2005. Available online: https://www.drugbank.ca/drugs/DB01053 (accessed on 8 October 2019).

39. DrugBank. Streptomycin. 2005. Available online: https://www.drugbank.ca/drugs/DB01082 (accessed on 8 October 2019).

40. Sinicina, I.; Pankratz, H.; Büttner, A.; Mall, G. Death due to neurogenic shock following gastric rupture in an anorexia nervosa patient. Forensic Sci. Int. 2005, 155, 7-12.

41. Arancibia, A.; Guttmann, J.; Gonzalez, G.; Gonzalez, C. Absorption and disposition kinetics of amoxicillin in normal human subjects. Antimicrob. Agents Chemother. 1980, 17, 199-202.

42. Tuano, S.B.; Johnson, L.D.; Brodie, J.L.; Kirby, W.M. Comparative blood levels of hetacillin, ampicillin and penicillin G. N. Engl. J. Med. 1966, 275, 635-639. 
43. Dailymed. Streptomycin Injection, Powder, lyophilized, for Solution. 2009. Available online: https://dailymed.nlm.nih.gov/dailymed/archives/fdaDrugInfo.cfm?archiveid=2439 (accessed on 8 October 2019).

44. Popeijus, H.E.; van Otterdijk, S.D.; van der Krieken, S.E.; Konings, M.; Serbonij, K.; Plat, J.; Mensink, R.P. Fatty acid chain length and saturation influences PPAR $\alpha$ transcriptional activation and repression in HepG2 cells. Mol. Nutr. Food Res. 2014, 58, 2342-2349. 


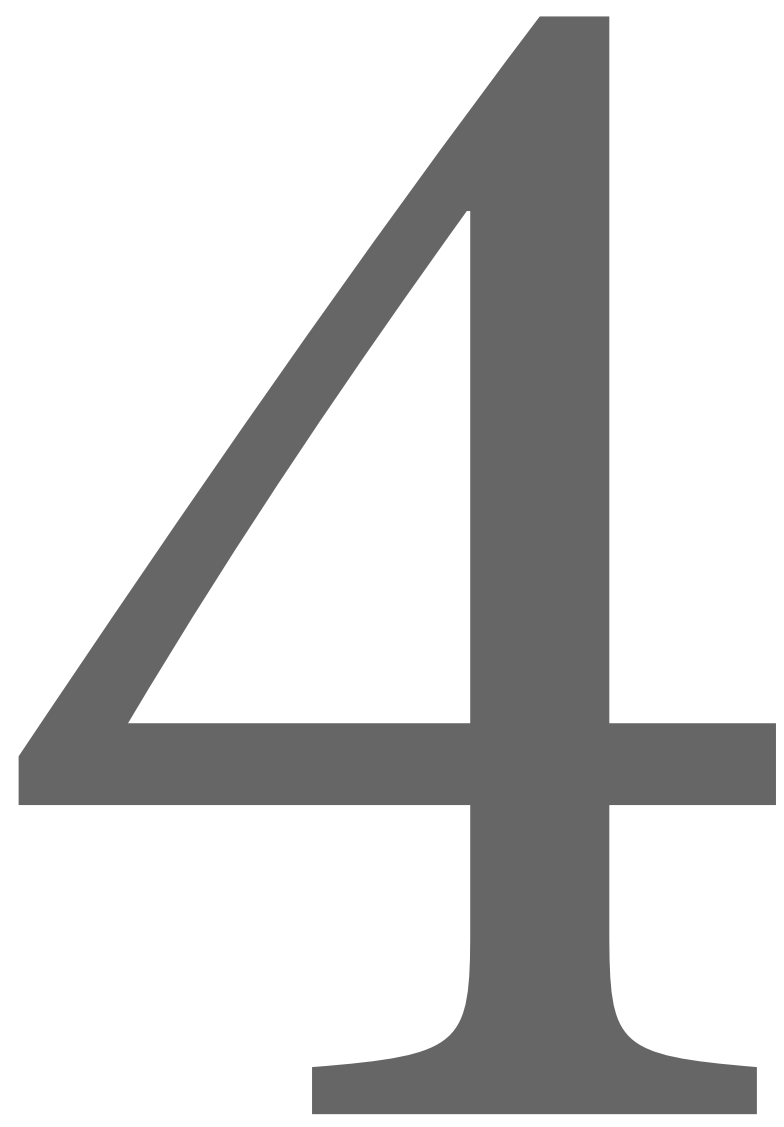




\section{Short-chain fatty acids (except Hexanoic acid) lower NF-kB transactivation, which rescues inflammation-induced decreased Apolipoprotein A-I transcription in HepG2 cells}

Jehad Z. Tayyeb | Herman E. Popeijus | Ronald P. Mensink | Maurice C.J.M. Konings | Fatma B.A. Mokhtar | Jogchum Plat 


\section{Abstract}

Concentrations of apolipoprotein A-I (ApoA-I) decrease during inflammation, which may lead to dysfunctional ApoA-I-poor high-density lipoprotein (HDL) particles, and as such, elevate cardiovascular risk. Therefore, rescuing ApoA-I concentrations, especially during inflammation, seems beneficial. Recently, shortchain fatty acids (SCFAs) have received more attention as a strategy in reversing atherosclerosis. We here evaluated the effects of SCFAs on inflammatory pathways in relation to ApoA-I transcription. SCFAs dose-response studies were performed in the presence and absence of inflammatory cytokines. ApoA-I and interleukin 8 (IL -8) mRNA expression were analyzed using qPCR and ELISA, respectively. To study underlying mechanisms, nuclear factor kappa $\mathrm{B}(\mathrm{NF}-\kappa \mathrm{B})$ transactivation and changes in mRNA expressions of the genes targets of bromodomain and extra-terminal (BET) inhibition, peroxisome proliferator-activated receptor-alpha (PPAR $\alpha$ ) transactivation and activator protein 1 (AP -1) pathway were analyzed. SCFAs (except hexanoic acid) increased ApoA-I mRNA transcription in both normal and inflammatory conditions and lowered IL-8 mRNA expression. This anti-inflammatory effect of SCFAs was confirmed by inhibition of NF- $\kappa \mathrm{B}$ transactivation. Moreover, butyric acid increased carnitine palmitoyltransferase 1 (CPT1), PPARa target gene, mRNA transcription in both conditions, and there was a negative correlation between CPT1 and NF- $\kappa$ B. Therefore, PPARa transactivation is probably involved in the anti-inflammatory effects of SCFAs, which rescues ApoA-I transcription. In conclusion, propionate, butyrate and valerate elicit anti-inflammatory effects which might rescue ApoA-I transcription in inflammatory conditions via PPARa transactivation mediated NF$\kappa \mathrm{B}$ inhibition. 


\section{Introduction}

Inflammation has clear effects on lipid and lipoprotein metabolism, which contributes to the association between inflammation and increased cardiovascular risk as seen for example in metabolic syndrome (1). It is known that inflammation strongly reduces high-density lipoprotein (HDL) cholesterol and particle concentrations and, more importantly, introduces the formation of dysfunctional HDL particles which consequently leads to impaired reverse cholesterol transport (2). Therefore, elevation of HDL particle concentration and more particularly its functionality, especially in inflamed conditions, might help to prevent the development of atherosclerosis and cardiovascular diseases (CVD) (3). The potential antiatherogenic effects of HDL have been linked to its main functional and structural protein, apolipoprotein A-I (ApoA-I) (4). Amongst others, ApoA-I is the acceptor for ATP-binding cassette transporter (ABCA1)-mediated cholesterol efflux and as such, regulates cholesterol efflux capacity (5). In addition, ApoA-I has many other functional and cardioprotective effects such as blunting inflammation (6) and lowering coagulant activity $(7,8)$. This explains the cross-sectional associations between ApoA-I and lower CVD risk. Lately, short chain fatty acids (SCFAs) have received more attention as they may be an attractive strategy in reversing the pathophysiology of metabolic disorders such as chronic inflammation and atherosclerosis (9). SCFAs are either absorbed and utilized by gut epithelial cells or transported directly to the liver via the portal vein (10). We have shown earlier that exposure to SCFAs elevates in vitro ApoA-I production in HepG2 cells (11). Moreover, Bartolomaeus and coworkers have recently shown that propionate (C3) supplementation reduced atherosclerosis in experimental animal models (12). This is in line with earlier observations suggesting a protective role for SCFAs in CVD development and inflammatory diseases (13). Furthermore, SCFAs can inhibit the proliferation and activation of T-cells and block the adhesion of antigen presenting cells in obesity-associated systemic inflammation (14). Given these effects of SCFAs on inflammation, here we evaluated the effects of SCFAs on the inflammatory pathways in relation to ApoA-I transcription in inflamed HepG2 cells and attempted to unravel the mechanism underlying these effects.

\section{Results}

Effects of SCFAs on ApoA-I mRNA expression in normal and inflammatory conditions

ApoA-I mRNA expression in HepG2 cells was lower under inflammatory conditions as compared to normal conditions (Figure 4.1). In agreement with our previous studies (15), the 
positive control JQ 1(+) and the negative control thapsigargin (Thap) respectively increased and decreased ApoA-I mRNA expression (both $\mathrm{p}<0.001$ ). Also, under inflammatory conditions, JQ1 $(+)$ significantly $(\mathrm{p}<0.001)$ increased ApoA-I gene expression, whereas Thap even further decreased ApoA-1 gene expression $(\mathrm{p}<0.001)$ compared with the normal condition (Figure 4.1). For the SCFAs, we observed that both under normal and inflammatory conditions, ApoA-I mRNA expression dose-dependently increased after C3, butyric acid (C4) and valeric acid (C5) treatment ( $\mathrm{p}<0.05)$, whereas hexanoic acid (C6) did not change ApoA-I mRNA expression. Interestingly, all SCFAs (except C6) were able to rescue the reduced levels of ApoA-I mRNA under the inflammatory condition. Both C3 and C5 fully rescued ApoA-I mRNA expression at $5.5 \mathrm{mM}$, and even increased ApoA-I mRNA expression at $7 \mathrm{mM}$, while C4 partly rescued ApoA-I mRNA expression up to 80\% at $4 \mathrm{mM}$ (Figure 4.1).
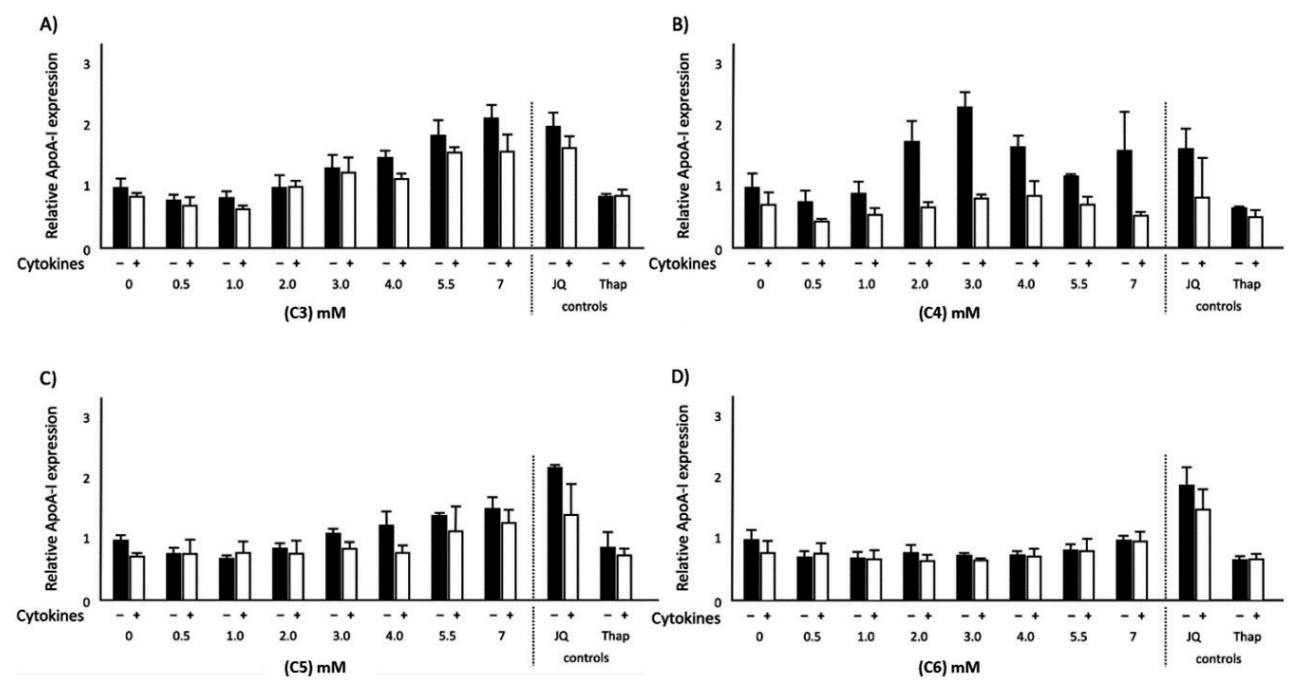

Figure 4.1. Relative apolipoprotein A-I (ApoA-I) mRNA expression in HepG2 cells treated with different concentrations of short chain fatty acids (SCFAs), JQ $1(+)(3 \mu \mathrm{M})$ or thapsigargin (Thap) $(0.01 \mu \mathrm{M})$. Six biological (12 technical) replicates were performed for every condition. (A) Increasing C3 concentrations showed a significant increase in ApoA-I mRNA expression in both normal and inflammatory conditions $(\mathrm{p}<0.05)$. (B) Increasing C4 concentrations showed a significant increase in ApoA-I mRNA expression in both normal and inflammatory conditions $(\mathrm{p}<0.05)$. (C) Increasing C5 concentrations showed a significant increase in ApoA-I mRNA expression in both normal and inflammatory conditions $(\mathrm{p}<0.05)$. (D) Increasing C6 concentrations did not show any significant effects in ApoA-I mRNA expression in both normal and inflammatory conditions. The positive control JQ1(+) and the negative control Thap respectively increased and decreased ApoA-I mRNA expression $(\mathrm{p}<0.001)$ in both normal and inflammatory conditions. All results are presented as the mean, while error bars indicate standard deviations. Data were normalized against the expression observed in the control condition, which was arbitrarily set at 1. Linear regression for SCFA doseresponse effects was performed except for $\mathrm{C} 4$, where a quadratic polynomial regression was performed to evaluate the dose-response effects. Changes were considered significant when the regression coefficients were significantly different from zero $(\mathrm{p}<0.05)$. 


\section{Effects of C4 on KEAP1 and CPT1 mRNA expression and NF- $\mathrm{kB}$ transactivation in normal and inflammatory conditions}

To understand the possible mechanism underlying how $\mathrm{C} 3, \mathrm{C} 4$ and $\mathrm{C} 5$ rescued the reduced ApoA-I mRNA during inflammation, we first explored the effects of $\mathrm{C} 4$ on kelch-like ECHassociated protein 1 (KEAP1) and carnitine palmitoyltransferase 1 (CPT1) mRNA gene expressions and nuclear factor kappa $\mathrm{b}(\mathrm{NF}-\kappa \mathrm{B})$ transactivation. $\mathrm{C} 4$ dose-dependently increased KEAP1 mRNA expression $(\mathrm{p}<0.05)$ under the inflammatory condition, while KEAP1 mRNA expression was reduced in the normal condition $(\mathrm{p}<0.05)$. Furthermore, CPT1 mRNA expression dose-dependently increased ( $\mathrm{p}<0.001)$ after C4 treatment, both under normal and inflammatory conditions (Figure 4.2). When cells were transfected with
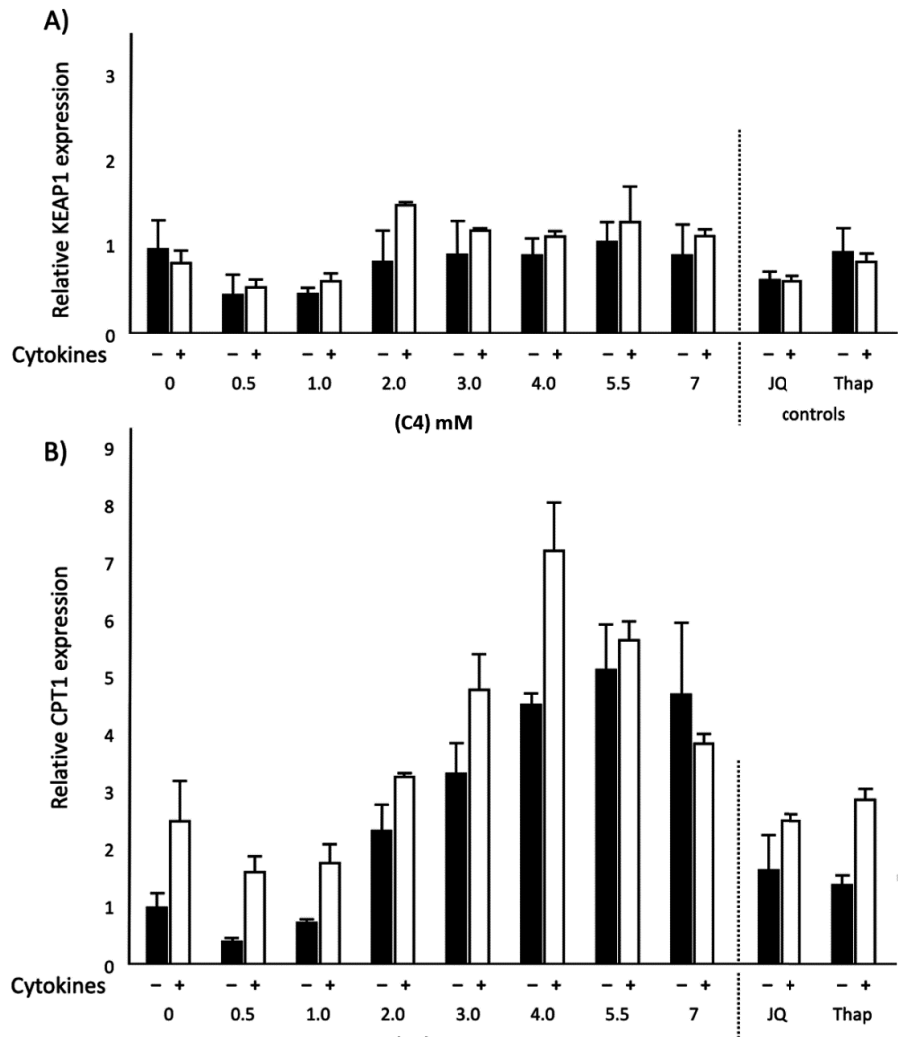

Figure 4.2. Relative kelch-like ECH-associated protein 1 (KEAP1) and carnitine palmitoyltransferase 1 (CPT1) mRNA expressions in HepG2 cells treated with different concentrations of C4, JQ1(+) $(3 \mu \mathrm{M})$ or thapsigargin (Thap) $(0.01 \mu \mathrm{M})$. (A) Increasing C4 concentrations showed a significant reduction in KEAP1 mRNA expression in the normal condition $(\mathrm{p}<0.05)$. Increasing $\mathrm{C} 4$ concentrations showed a significant increase in KEAP1 mRNA expression in the inflammatory condition $(\mathrm{p}<0.05)$. (B) Increasing C4 concentrations showed a significant increase in CPT1 mRNA expression in both normal and inflammatory conditions $(p<0.001)$. All results are presented as the mean, while error bars indicate standard deviations. Data were normalized against the expression observed in the control condition, which was arbitrarily set at 1 . A quadratic polynomial regression was performed for $\mathrm{C} 4$ dose-response effects. Changes were considered significant when the regression coefficients were significantly different from zero $(p<0.05)$. 
the NF- $\kappa \mathrm{B}$ reporter, both in normal as well as inflammatory conditions, C4 significantly $(\mathrm{p}<0.05)$ decreased NF- $\kappa \mathrm{B}$ transactivation (Figure 4.3). Moreover, an inverse correlation was found between CPT1 mRNA expression and NF- $\kappa$ B transactivation in inflamed HepG2 cells after $\mathrm{C} 4$ treatment $(\mathrm{r}=-0.733$; $\mathrm{p}<0.05)$. Finally, looking at the effects of the positive and negative controls, as expected, the bromodomain and extra-terminal (BET) inhibitor JQ1(+) significantly decreased KEAP1 gene expression $(\mathrm{p}<0.05)$ both under inflammatory and normal conditions, whereas Thap did not affect KEAP1 gene expression. Furthermore, both JQ1 $(+)$ and Thap significantly $(\mathrm{p}<0.05)$ increased CPT1 gene expression in both conditions (Figure 4.2).
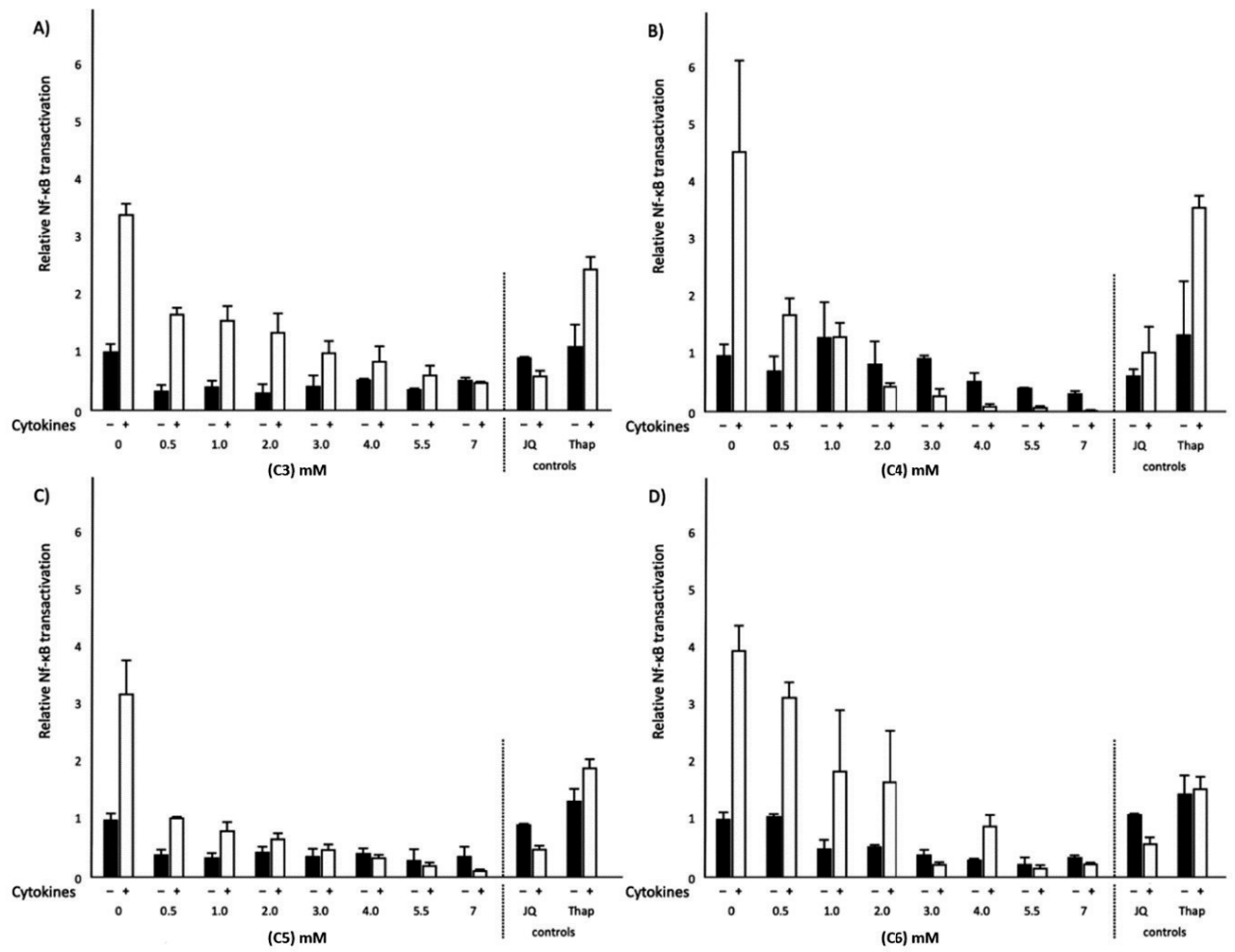

Figure 4.3. Relative nuclear factor kappa $\mathrm{b}(\mathrm{NF}-\mathrm{\kappa B})$ transactivation in HepG2 cells treated with different concentrations of SCFAs, JQ1 $(+)(3 \mu \mathrm{M})$ or thapsigargin (Thap) $(0.01 \mu \mathrm{M})$. (A) Increasing C3 concentrations showed a significant decrease in NF- $\kappa B$ transactivation in the inflammatory condition $(\mathrm{p}<0.05)$. (B) Increasing C4 concentrations showed a significant decrease in NF- $\mathrm{kB}$ transactivation in both normal and inflammatory conditions $(\mathrm{p}<0.05)$. (C) Increasing $\mathrm{C} 5$ concentrations showed a significant decrease in NF- $\kappa B$ transactivation in the inflammatory condition $(\mathrm{p}<0.05)$. (D) Increasing C6 concentrations showed a significant decrease in NF- $\kappa B$ transactivation in both normal and inflammatory conditions $(p<0.05)$. All results are presented as the mean, while error bars indicate standard deviations. Data were normalized against the transactivation observed in the control condition, which was arbitrarily set at 1 . Linear regression for SCFA dose-response effects was performed except for $\mathrm{C} 4$, where a quadratic polynomial regression was performed to evaluate the dose-response effects. Changes were considered significant when the regression coefficients were significantly different from zero $(\mathrm{p}<0.05)$. 


\section{Effects of different SCFAs on NF-אB transactivation and IL-8 secretion in normal and inflammatory conditions}

To extend the observed effects of $\mathrm{C} 4$ on NF- $\mathrm{kB}$ transactivation, the above-mentioned experiments for $\mathrm{C} 4$ were repeated with $\mathrm{C} 3, \mathrm{C} 5$ and $\mathrm{C} 6$. In the normal condition, not only C4 but also C6 significantly decreased NF- $\kappa$ B transactivation $(\mathrm{p}<0.05)$, whereas C3 and $\mathrm{C} 5$ did not have any effects on NF- $\kappa \mathrm{B}$ transactivation. In the inflammatory condition, all SCFAs significantly $(\mathrm{p}<0.05)$ decreased NF- $\kappa \mathrm{B}$ transactivation (Figure 4.3). Moreover, all SCFAs studied did not affect interleukin 8 (IL -8) secretion in normal conditions. On the other hand, in the inflammatory condition, C3, C4 and C5 significantly ( $p<0.05)$ decreased IL-8 secretion (Figure 4.4). Interestingly, C6 did not lower IL-8 secretion even
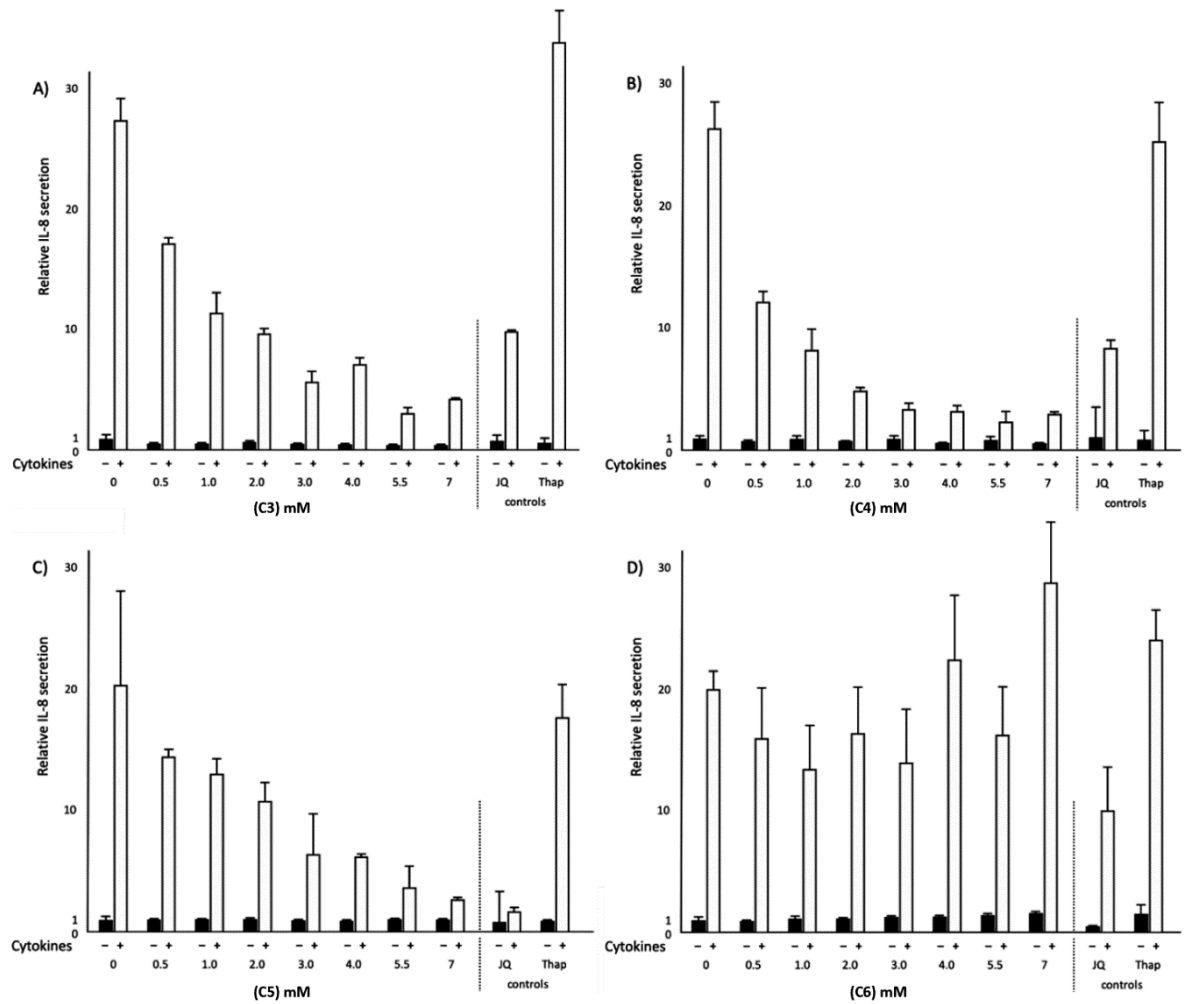

Figure 4.4. Relative interleukin 8 (IL-8) secretion in HepG2 cells treated with different concentrations of SCFAs, JQ1 $(+)(3 \mu \mathrm{M})$ or thapsigargin (Thap) $(0.01 \mu \mathrm{M})$. (A) Increasing C3 concentrations showed a significant decrease in IL-8 secretion in the inflammatory condition $(\mathrm{p}<0.05)$. (B) Increasing C4 concentrations showed a significant decrease in IL-8 secretion in the inflammatory condition $(\mathrm{p}<0.05)$. (C) Increasing C5 concentrations showed a significant decrease in IL-8 secretion in the inflammatory condition $(\mathrm{p}<0.05)$. (D) Increasing C6 concentrations did not show any significant effects on IL-8 secretion in either normal or inflammatory conditions. All results are presented as the mean, while error bars indicate standard deviations. Data were normalized against secretion observed in the control condition, which was arbitrarily set at 1 . Linear regression for SCFAs dose-response effects was performed except for $\mathrm{C} 4$, where a quadratic polynomial regression was performed to evaluate the dose-response effects. Changes were considered significant when the regression coefficients were significantly different from zero $(\mathrm{p}<0.05)$. 
though C6 lowered NF- $\kappa$ B transactivation. JQ1(+) did not affect NF- $\kappa \mathrm{B}$ transactivation, whereas Thap significantly $(\mathrm{p}<0.05)$ increased NF- $\kappa \mathrm{B}$ transactivation in both conditions (Figure 4.3). Finally, both JQ1 $(+)$ and Thap significantly $(\mathrm{p}<0.05)$ further increased IL-8 secretion under the inflammatory condition, but these effects were not observed under the normal condition (Figure 4.4).

\section{Effects of different SCFAs on c-Jun and c-Fos mRNA expression in normal and inflammatory conditions}

To further explore the role of $\mathrm{C} 3, \mathrm{C} 4$ and $\mathrm{C} 5$ in rescuing ApoA-I transcription during inflammation, we also examined - besides NF- $\kappa$ B transactivation - the effects of SCFAs
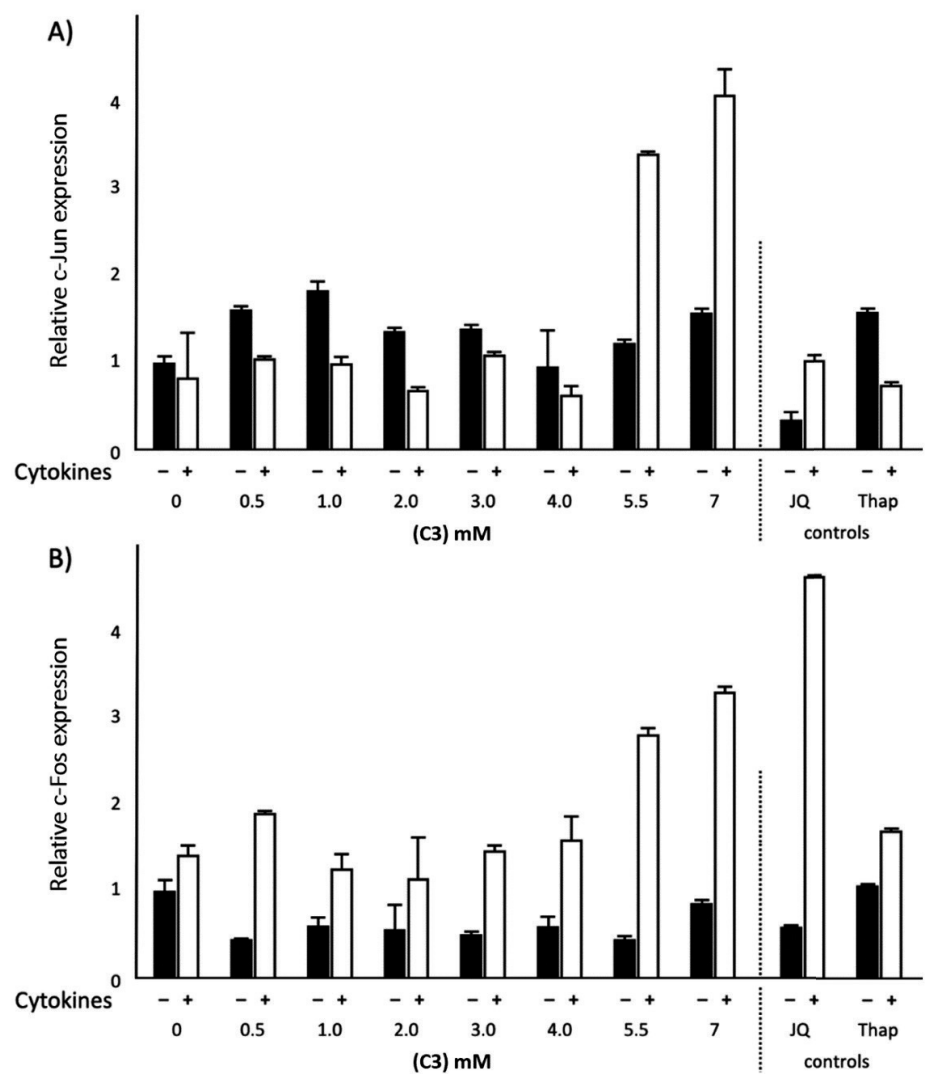

Figure 4.5. Relative c-Jun and c-Fos mRNA expressions in HepG2 cells treated with different concentrations of C3, JQ1 $(+)(3 \mu \mathrm{M})$ or thapsigargin (Thap) $(0.01 \mu \mathrm{M})$. (A) Increasing C3 concentrations showed a significant increase in c-Jun mRNA expression in the inflammatory condition ( $\mathrm{p}<0.01)$. (B) Increasing C3 concentrations showed a significant increase in c-Fos mRNA expression in the inflammatory condition $(\mathrm{p}<0.01)$. All results are presented as the mean, while error bars indicate standard deviations. Data were normalized against the expression observed in the control condition, which was arbitrarily set at 1 . A linear regression analysis to evaluate $\mathrm{C} 3$ dose-response effects was performed. Changes were considered significant when the regression coefficients were significantly different from zero $(\mathrm{p}<0.05)$. 
on activation of the activator protein (AP-1) pathway by analyzing potential changes in c-Fos and c-Jun mRNA expression. If the AP-1 pathway was involved, we would expect a reduction in AP-1 activation as translated into lower c-Fos and c-Jun expression. In normal conditions, all SCFAs (except C3) significantly $(\mathrm{p}<0.01)$ increased c-Jun mRNA expression. In the inflammatory condition, all SCFAs increased $(\mathrm{p}<0.01) \mathrm{c}$-Jun mRNA expression. Furthermore, all SCFAs did not change c-Fos mRNA expression in the normal condition, except C4, which significantly increased $(\mathrm{p}<0.001) \mathrm{c}$-Fos mRNA expression. Moreover, all SCFAs increased $(\mathrm{p}<0.01) \mathrm{c}$-Fos mRNA expression in the inflammatory condition, except C6, which significantly decreased $(\mathrm{p}<0.05)$ c-Fos mRNA expression. Effects of C3 are shown in (Figure 4.5), whereas the effects of all SCFAs are shown in Supplementary Table S4.1.

\section{Discussion}

The SCFAs propionic acid (C3), butyric acid (C4), valeric acid (C5) and hexanoic acid (C6) are produced after the fermentation of dietary fibers and resistant starches by the microbiota in the cecum and colon (9). An increasing number of functions and beneficial effects of SCFAs on human metabolism have been described (14). Amongst others, SCFAs have anti-inflammatory effects and modulate different processes including cell proliferation, hormone secretion and immune responses (16). SCFAs are either absorbed and utilized by gut epithelial cells or transported directly to the liver via the portal vein (10). Despite different reports describing the beneficial effects of SCFAs in HepG2 cells, the effects of SCFAs on human liver cells under inflammatory conditions have not been studied. We recently described a favorable effect of SCFAs on ApoA-I mRNA transcription (11), but these effects were observed in normal (non-inflamed) HepG2 cells. Exposure to a cocktail of inflammatory cytokines of tumor necrosis factor alpha (TNF- $\alpha$ ) and interleukin 1 beta (IL-1 $\beta$ ) has already been shown to decrease ApoA-I mRNA levels in HepG2 cells (17). Here we report the effects of SCFAs in HepG2 cells exposed to these cytokines, in which ApoA-I mRNA transcription was indeed lower due to the inflammatory response. This lower ApoA-I transcription in cytokine-exposed cells is in line with the known effects of acute phase responses on lipoprotein metabolism $(18,19)$.

We observed that all four SCFAs studied here have anti-inflammatory effects as shown by a lower NF- $\kappa \mathrm{B}$ transactivation, which in turn (except for C6) rescued the inflammationinduced reduced hepatic ApoA-I mRNA expression. This seems to be a logical response since the ApoA-I gene contains several NF- $\kappa B$ binding sites in the promoter region in hepatocytes $(20,21)$. The anti-inflammatory effects of C3, C4 and C5 were confirmed by a 
reduction in IL-8 secretion into the supernatant of the cells. However, C6 did not affect IL-8 secretion in the inflamed hepatic cells, which was remarkable since C6 did lower NF- $\mathrm{B}$ transactivation just like the three other SCFAs.

These findings for these SCFAs were in line with previous studies which also evaluated the relation between SCFA exposure and inflammation in other cells types. For example, Qiao et al. (22) showed that C4 inhibited TNF- $\alpha$, IL- 6 and myeloperoxidase activity by preventing NF- $\kappa B$ transactivation in the liver cells of Sprague-Dawley rats. Another study showed that both C4 and C3 reduced IL-6 and IL-8 levels in human umbilical vein endothelial cells that were stimulated with lipopolysaccharide (LPS) and TNF- $\alpha$ (23). Moreover, Usami and coworkers showed that $\mathrm{C} 3$ and $\mathrm{C} 4$ both reduced TNF- $\alpha$ production and downregulated $\mathrm{NF}-\kappa \mathrm{B}$ transactivation in peripheral blood mononuclear cells (24). Here we extend these observations of C3 and C4 to C5 in HepG2 cells and link this interesting finding to the rescued expression of ApoA-I during inflammation.

To investigate the mechanisms underlying the rescued ApoA-I transcription by SCFA treatment in relation to the observed reduced NF- $\kappa \mathrm{B}$ transactivation in more detail, changes in markers for BET inhibition and PPARa transactivation were analyzed. This analysis was conducted via testing BET and PPAR $\alpha$ target gene expression (KEAP1 and $\mathrm{CPT} 1$, respectively). In both normal and inflammatory conditions, $\mathrm{C} 4$ treatment increased CPT1 mRNA transcription, whereas KEAP1 mRNA transcription was decreased in the normal condition only. Furthermore, we found a significant negative correlation between CPT1 mRNA expression and NF- $\kappa$ B transactivation associated with $\mathrm{C} 4$ treatment in the inflammatory condition. Consequently, this finding suggests the ability of PPAR $\alpha$ to reduce inflammation by inhibiting NF- $\kappa B$ transactivation. All together, these results of $C 4$ suggested that PPARa transactivation might be involved in the effects of SCFAs on ApoA-I expression in normal and inflammatory conditions. This is also in agreement with our previous study in which we found a clear role for PPARa transactivation on ApoA-I mRNA transcription in non-inflamed HepG2 cells (15). Moreover, it seems that BET inhibition does not contribute to the effects of SCFAs on ApoA-I during inflammatory conditions, since KEAP1 was not reduced in inflamed HepG2 cells.

We observed variations in the ability of SCFAs to increase ApoA-I expression or to reduce IL-8 secretion. In our previous study in non-inflamed conditions (11), we have shown that C6 was the weakest inducer of ApoA-I mRNA expression as compared to the other SCFAs (C3, C4 and C5). Again, in the present study, we found that C6 was the only SCFA that was unable to rescue ApoA-I transcription in the inflammatory condition. Moreover, although $\mathrm{C} 6$ reduced $\mathrm{NF}-\kappa \mathrm{B}$ transactivation just like the other SCFAs, we found that C6 
did not inhibit IL-8 secretion like the other SCFAs. This outcome regarding the effects of C6 suggests that the anti-inflammatory effects of SCFAs related to ApoA-I might not be linked only to a blunted NF- $\mathrm{\kappa B}$ transactivation but could additionally also be ascribed to other (inflammatory) pathways. Since the observed reduction in IL-8 was associated with increased ApoA-I expression after C3, C4 and C5 treatment, while C6 did not lower IL-8 expression and also did not elevate ApoA-I expression, we speculated that the ApoA-I rescue effects of $\mathrm{C} 3, \mathrm{C} 4$ and $\mathrm{C} 5$ could also be the result of another modulating anti-inflammatory signaling pathway underlying IL-8 secretion. The promoter region of the IL-8 gene contains not only functional binding sites for NF- $\mathrm{kB}$, but also for AP- 1 and CCAAT/enhancer binding protein $\beta$ (C/EBP- $\beta$ ) (25). It has been described that PPAR $\alpha$, which is known as a regulatory factor of ApoA-I (15), is also linked to the AP-1 pathway (26). PPAR $\alpha$ transactivation had an inhibitory effect on both NF- $\mathrm{KB}$ and AP-1, which led to the inhibition of inflammation (26). Although an earlier study showed a significant correlation between SCFA exposure and the regulation of the AP-1 signaling pathway in intestinal cells (27), the relationship between SCFAs and the AP-1 pathway in inflamed liver cells has not been studied before. Consequently, we decided to investigate the antiinflammatory effects of SCFAs in relation to the AP-1 signaling pathway as an additional candidate besides lowering NF- $\mathrm{kB}$ pathway activity. Therefore, the mRNA expression of c-Jun and c-Fos, both target genes of the AP-1 pathway, were evaluated in both normal and inflamed HepG2 cells. Unfortunately, all SCFAs, again except C6, did not inhibit the AP-1 signaling pathway in the inflammatory condition. This means that inhibition of the AP-1 pathway was probably not involved in the anti-inflammatory effects of those SCFAs that were able to elevate ApoA-I mRNA expression. However, since the transcriptional activity of c-Jun is regulated mainly post-transcriptionally via phosphorylation, other types of experiments need to be performed to confirm the absence of AP-1 involvement. Altogether, our findings regarding the relationship between exposure to SCFAs and NF- $\mathrm{\kappa B}$ or AP-1 pathway activation allow us to speculate that maybe the third candidate regulator C/EBP- $\beta$ with a binding site in the IL- 8 promoter might be a potential mediator involved in the effects of SCFAs during inflammation. Indeed, Bai and coworkers have shown that overexpression of C/EBP- $\beta$ increased the expression of cytokines such as IL-8 (28). Furthermore, the ApoA-I promoter has a C/EBP binding site, which indicates that C/EBP- $\beta$ might be involved in ApoA-I production (29). As a result, we suggest in future experiments to investigate the role of SCFAs in rescuing the inflammation-induced reduction in ApoA-I expression by evaluating their effects on the C/EBP- $\beta$ signaling pathway.

For now, we conclude that the SCFAs propionic acid, butyric acid and valeric acid can reduce NF- $\kappa \mathrm{B}$ mediated pro-inflammatory responses, probably mediated via PPAR $\alpha$ 
transactivation, which translates into a rescue of ApoA-I transcription in inflamed HepG2 cells. This anti-inflammatory effect of C3, C4 and C5 was confirmed by the inhibition of both NF- $\kappa$ B transactivation and IL-8 secretion.

\section{Material and methods}

\section{Materials}

Human hepatocellular liver carcinoma cells (HepG2) were kindly provided by Sten Braesch-Andersen (Mabtech, Nacka Strand, Sweden). Cell culture flasks and plates were obtained from Corning (Corning, NY, USA). Minimum Essential Medium (MEM), sodium pyruvate, non-essential amino acids (NEAA), penicillin and streptomycin were all obtained from Thermo Fisher Scientific (Bleiswijk, Netherlands). Fetal bovine serum (FBS) was purchased from PAA (Toronto, ON, Canada). Propionic acid (C3), butyric acid (C4), valeric acid (C5) and hexanoic acid (C6) were bought from Sigma (Uithoorn, Netherlands). JQ1(+) was obtained from Bio-Techne R\&D (Minneapolis, MN, USA). Tumor necrosis factor-alpha (TNF- $\alpha$ ), interleukin-1 beta (IL-1 $\beta$ ), thapsigargin (Thap), dimethyl sulfoxide (DMSO) and Tri-reagent were purchased from Sigma (Uithoorn, Netherlands).

\section{Cell culture and SCFA treatment}

HepG2 cells were cultured at $37^{\circ} \mathrm{C}$ in a humidified atmosphere of $5 \%$ carbon dioxide $\left(\mathrm{CO}_{2}\right)$ in MEM containing $10 \%$ heat inactivated FBS, $1 \%$ sodium pyruvate, $1 \%$ NEAA and $1 \%$ of penicillin-streptomycin mixture. For all experiments, cells were seeded in a 24-well plate at a density of 200,000 cells per well. Cell viability was inspected daily by microscope and when cells reached a density of $80-90 \%$, they were incubated for $48 \mathrm{~h}$ in the medium (MEM without FBS) plus a concentration range of $0-7$ mM SCFAs (C3, C4, C5 or C6) or $3 \mu \mathrm{M}$ JQ1(+) with or without a cytokine cocktail (TNF- $\alpha 100 \mathrm{ng} / \mathrm{mL}$ and IL-1 $\beta 5 \mathrm{ng} / \mathrm{mL}$ ). A positive control JQ1(+), a BET inhibitor, was included in each experiment to ensure that cells were responsive and produced sufficient amounts of ApoA-I mRNA. Thapsigargin (Thap), an endoplasmic reticulum (ER )stress inducer, was used as a negative control. All SCFAs, JQ1(+) and Thap were dissolved in dimethyl sulfoxide (DMSO, cell culture tested) and effects were expressed relative to those of the carrier control (DMSO only). The final DMSO concentration was always $0.2 \%$. Culture medium was collected for the analysis of IL-8 concentrations and cells were harvested for the analysis of mRNA expression after lysing with Tri-reagent. Both culture medium and lysed cells were snap frozen in liquid nitrogen and stored at $-80^{\circ} \mathrm{C}$ until further analysis. 


\section{Quantification of gene mRNA transcription}

To evaluate effects of SCFAs on mRNA expression levels of ApoA-I, KEAP1, CPT1, c-Jun and c-Fos, total RNA was isolated from HepG2 cells using Tri-reagent according to the manufacturer's instructions. The RNeasy Mini Kit (Qiagen, Hilden, Germany) was used for RNA purification. For cDNA synthesis, 350 ng of total RNA was reverse-transcribed using RNAse inhibitor, dNTPs, random hexamers, moloney murine leukemia virus (MMLV) reverse trans, Dithiothreitol (DTT) and 5x reverse transcriptase (RT) buffer (Thermo Fisher Scientific, Bleiswijk, Netherlands). The resulting cDNA was used for real-time quantitative PCR using TaqMan Gene Expression Assays using cyclophilin A (Hs99999904) as a housekeeping control. To quantify ApoA-I, KEAP1, CPT1, c-Jun and c-Fos, the TaqMan gene expression assays Hs00163641, Hs00202227, Hs00912671, Hs00277190 and Hs00170630 were used. Values are presented as relative gene expressions based on the cycle threshold $(\mathrm{Ct})$ values, normalized for the internal control cyclophilin $\mathrm{A}$, and compared to the control conditions.

\section{Luciferase assay}

To investigate effects of SCFAs on NF- $\mathrm{BB}$ transactivation, HepG2 cells were transfected with X-treme gene 9 DNA transfection reagent (Sigma, Uithoorn, Netherlands) and the plasmids pcDNA3.1, pGL3 and NF- $\kappa B$ pGL3 following the manufacturer's instructions. The pcDNA3.1 (empty vector) and the empty pGL3 luciferase reporter (without NF- $\kappa \mathrm{B}$ elements) were used as a negative control. Following transfection and $48 \mathrm{~h}$ SCFA treatment with or without the cytokine cocktail, cells were lysed in luciferase lysis buffer (Promega, Madison, WI, USA) and measured for luciferase activity, reflecting NF- $\kappa$ B transactivation, using a GloMax 96 Microplate luminometer, according to the manufacturer's manual (Promega, Madison, WI, USA).

\section{Quantification of IL-8 secretion levels in the culture medium}

To investigate IL-8 secretion by HepG 2 cells, IL- 8 protein concentrations in culture medium were measured by an enzyme-linked immunoassay (ELISA; Hycult Biotechnology, Uden, The Netherlands) following the manufacturer's instructions.

\section{Statistical analysis}

All independent dose-response experiments with the SCFAs were performed in duplicate and each experiment was repeated three times. Six biological (12 technical) replicates were performed for every single SCFA dose. Regression analysis was used to examine the relationships between SCFAs and the parameters. First, we modeled via quadratic polynomial 
regression, which fits with a parabolic relationship as seen for $\mathrm{C} 4$. In case this did not reach significance, a linear relationship (i.e., as seen for C3 and C5) was examined. Where mentioned, Spearman correlations were calculated. The regression coefficients and Spearman correlation coefficients were considered to be statistically significant when different from zero at $\mathrm{p}<0.05$. Effects of the positive JQ1(+) and negative (Thap) controls were statistically evaluated by Mann-Whitney $U$ test, in which a p-value $<0.05$ was considered statistically significant. All statistical analyses were performed using SPSS v.25 (IBM Corp., Armonk, NY, USA).

\section{References}

1. Esteve, E.; Ricart, W.; Fernández-Real, J.M. Dyslipidemia and inflammation: An evolutionary conserved mechanism. Clin. Nutr. 2005, 24, 16-31.

2. Kosmas, C.E.; Silverio, D.; Sourlas, A.; Montan, P.D.; Guzman, E. Dysfunctional high-density lipoprotein and atherogenesis. Vessel Plus. 2019, 3, 2.

3. Chyu, K.-Y.; Peter, A.; Shah, P.K. Progress in HDL-based therapies for atherosclerosis. Curr. Atheroscler. Rep. 2011, 13, 405.

4. Haas, M.; Reinacher, D.; Li, J.; Wong, N.; Mooradian, A. Regulation of apoA1 gene expression with acidosis: Requirement for a transcriptional repressor. J. Mol. Endocrinol. 2001, 27, 43-57.

5. Du, Y.; Wang, L.; Hong, B. High-density lipoprotein-based drug discovery for treatment of atherosclerosis. Expert. Opin. Drug. Dis. 2015, 10, 841-855.

6. Gerster, R.; Eloranta, J.J.; Hausmann, M.; Ruiz, P.A.; Cosin-Roger, J.; Terhalle, A. Anti-inflammatory function of high-density lipoproteins via autophagy of IкB kinase. Cell. Mol. Gastroenterol. 2015, 1, 171-187.

7. Oslakovic, C.; Krisinger, M.; Jauhiainen, M.; Ehnholm, C.; Dahlbäck, B. 614 anticoagulant properties of apolipoprotein ai and phospholipid transfer protein. Atheroscler. Suppl. 2009, 10, 138.

8. Georgila, K.; Vyrla, D.; Drakos, E. Apolipoprotein AI (ApoA-I), Immunity, Inflammation and Cancer. Cancers. 2019, 11, 1097.

9. Ohira, H.; Tsutsui, W.; Fujioka, Y. Are short chain fatty acids in gut microbiota defensive players for inflammation and atherosclerosis? J. Atheroscler. Thromb. 2017, 24, 660-672.

10. Nakatani, M.; Inoue, R.; Tomonaga, S.; Fukuta, K.; Tsukahara, T. Production, Absorption, and Blood Flow Dynamics of Short-Chain Fatty Acids Produced by Fermentation in Piglet Hindgut during the Suckling-Weaning Period. Nutrients. 2018, 10, 1220.

11. Tayyeb, J.Z.; Popeijus, H.E.; Mensink, R.P.; Konings, M.C.; Mulders, K.H.; Plat, J. The effects of short-chain fatty acids on the transcription and secretion of apolipoprotein A-I in human hepatocytes in vitro. J. Cell. Biochem. 2019, 120, 17219-17227.

12. Bartolomaeus, H.; Balogh, A.; Yakoub, M.; Homann, S.; Markó, L.; Höges, S. Short-chain fatty acid propionate protects from hypertensive cardiovascular damage. Circulation 2019, 139, 1407-1421.

13. Wong, J.M.; De Souza, R.; Kendall, C.W.; Emam, A.; Jenkins, D.J. Colonic health: Fermentation and short chain fatty acids. J. Clin. Gastroenterol. 2006, 40, 235-243.

14. Den Besten, G.; Van Eunen, K.; Groen, A.K.; Venema, K.; Reijngoud, D.-J.; Bakker, B.M. The role of short-chain fatty acids in the interplay between diet, gut microbiota, and host energy metabolism. J. Lipid Res. 2013, 54, 2325-2340. 
15. Van der Krieken, S.E.; Popeijus, H.E.; Mensink, R.P.; Plat, J. Link between ER-stress, PPAR-alpha activation, and BET inhibition in relation to apolipoprotein A-I transcription in HepG2 cells. J. Cell. Biochem. 2017, 118, 2161-2167.

16. Vinolo, M.A.; Rodrigues, H.G.; Nachbar, R.T.; Curi, R. Regulation of inflammation by short chain fatty acids. Nutrients 2011, 3, 858-876.

17. Haas, M.J.; Horani, M.; Mreyoud, A.; Plummer, B.; Wong, N.C.; Mooradian, A.D. Suppression of apolipoprotein AI gene expression in HepG2 cells by TNF $\alpha$ and IL-1 $\beta$. Biochim. Biophys. Acta 2003, 1623, 120-128.

18. Carpintero, R.; Pineiro, M.; Andres, M.; Iturralde, M.; Alava, M.; Heegaard, P.M. The concentration of apolipoprotein AI decreases during experimentally induced acute-phase processes in pigs. Infect. Immun. 2005, 73, 3184-3187.

19. Chait, A.; Han, C.Y.; Oram, J.F.; Heinecke, J.W. Lipoprotein-associated inflammatory proteins: Markers or mediators of cardiovascular disease. J. Lipid Res. 2005, 46, 389-403.

20. Morishima, A.; Ohkubo, N.; Maeda, N.; Miki, T.; Mitsuda, N. NFkB regulates plasma apolipoprotein $\mathrm{AI}$ and high density lipoprotein cholesterol through inhibition of peroxisome proliferator-activated receptor a. J. Biol. 2003, 278, 38188-38193.

21. Trusca, V.G.; Dumitrescu, M.; Fenyo, I.M.; Tudorache, I.F.; Simionescu, M.; Gafencu, A.V. The Mechanism of Bisphenol A Atherogenicity Involves Apolipoprotein AI Downregulation through NF- $\kappa B$ Activation. Int. J. Mol. Sci. 2019, 20, 6281.

22. Qiao, Y.-L.; Qian, J.-M.; Wang, F.-R.; Ma, Z.-Y.; Wang, Q.-W. Butyrate protects liver against ischemia reperfusion injury by inhibiting nuclear factor kappa B activation in Kupffer cells. J. Surg. Res. 2014, 187, 653-659.

23. Li, M.; van Esch, B.C.; Henricks, P.A.; Garssen, J.; Folkerts, G. Time and concentration dependent effects of short chain fatty acids on lipopolysaccharide-or tumor necrosis factor a-induced endothelial activation. Front. Pharmacol. 2018, 9, 233.

24. Usami, M.; Kishimoto, K.; Ohata, A.; Miyoshi, M.; Aoyama, M.; Fueda, Y. Butyrate and trichostatin A attenuate nuclear factor kappaB activation and tumor necrosis factor alpha secretion and increase prostaglandin E2 secretion in human peripheral blood mononuclear cells. Nutr. Res. 2008, 28, 321-328.

25. Elliott, C.; Allport, V.; Loudon, J.; Wu, G.; Bennett, P. Nuclear factor-kappa B is essential for upregulation of interleukin-8 expression in human amnion and cervical epithelial cells. Mol. Hum. Reprod. 2001, 7, 787-790.

26. Martin, G.; Duez, H.; Blanquart, C.; Berezowski, V.; Poulain, P.; Fruchart, J.-C. Statin-induced inhibition of the Rho-signaling pathway activates PPARa and induces HDL apoA-I. J. Clin. Investig. 2001, 107, 1423-1432.

27. Nepelska, M.; Cultrone, A.; Beguet-Crespel, F.; Le Roux, K.; Dore, J.; Arulampalam, V. Butyrate produced by commensal bacteria potentiates phorbol esters induced AP-1 response in human intestinal epithelial cells. PLoS One. 2012, 7, e52869.

28. Bai, Y.; Wei, Y.; Wu, L.; Wei, J.; Wang, X.; Bai, Y. C/EBP $\beta$ mediates endoplasmic reticulum stress regulated inflammatory response and extracellular matrix degradation in LPS-stimulated human periodontal ligament cells. Int. J. Mol. Sci. 2016, 17, 385.

29. Van der Krieken, S.E.; Popeijus, H.E.; Konings, M.; Dullens, S.P.; Mensink, R.P.; Plat, J. C/EBP- $\beta$ Is Differentially Affected by PPARa Agonists Fenofibric Acid and GW7647, But Does Not Change Apolipoprotein A-I Production During ER-Stress and Inflammation. J. Cell. Biochem. 2017, 118, 754-763. 


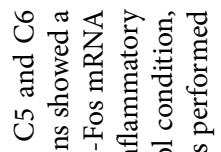

ऊी.



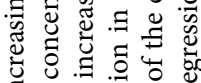

声青要

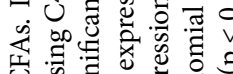

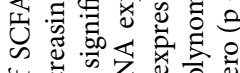

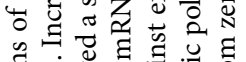

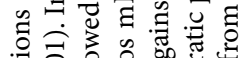

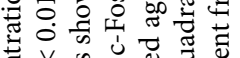

矛 $\vee$ :

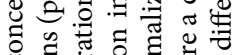

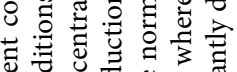

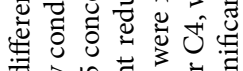

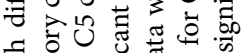

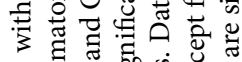

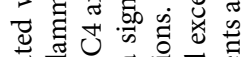

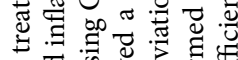

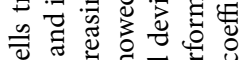



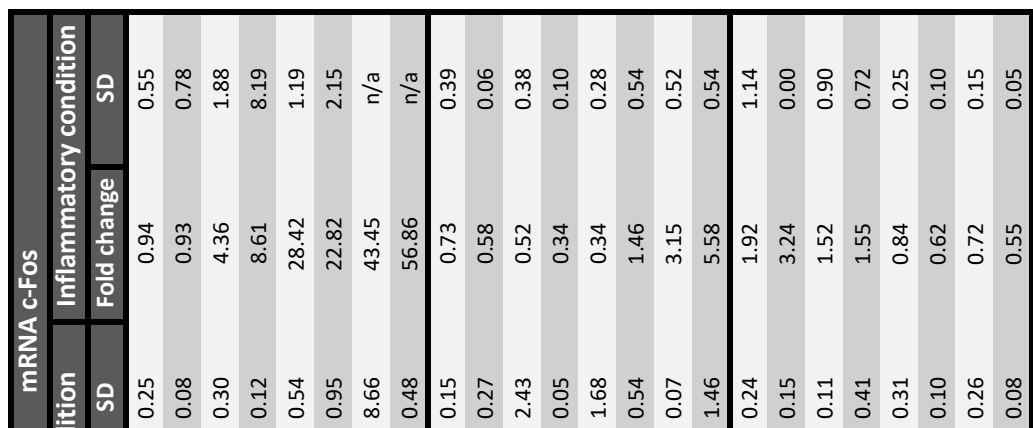

)

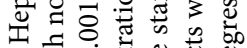

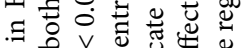

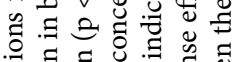

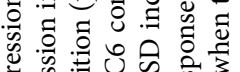

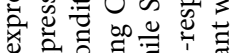

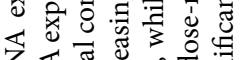



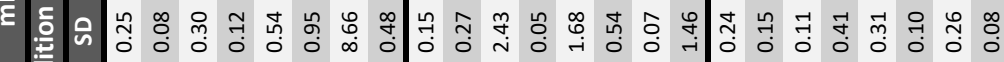

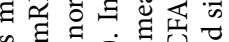

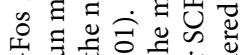

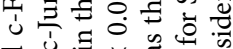

宅.

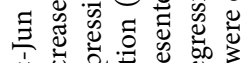

ن

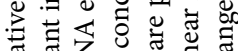

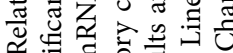

$\approx$ 纯

둘

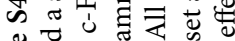



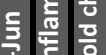

๓

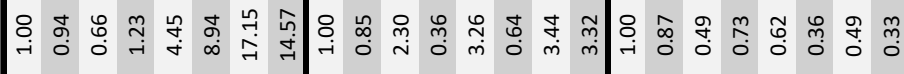

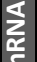

등

क:

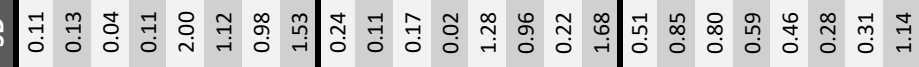

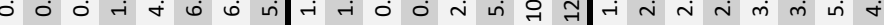

ती

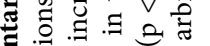

牙泀

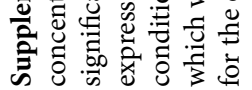




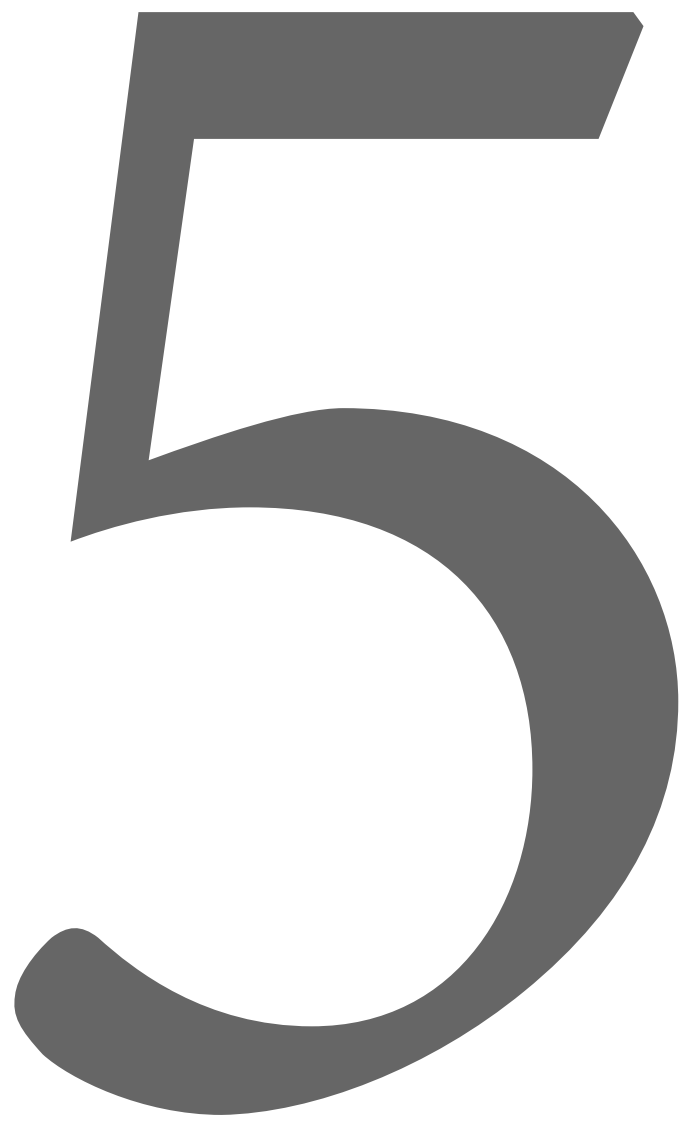




\section{Butyric acid added apically to intestinal Caco-2 cells elevates}

hepatic ApoA-I transcription and rescues lower ApoA-I expression in inflamed HepG2 cells co-cultured in the basolateral compartment

Jehad Z. Tayyeb | Herman E. Popeijus | Ronald P. Mensink | Jogchum Plat 


\section{Abstract}

Apolipoprotein A-I (ApoA-I) concentrations are decreased during inflammation, which may reduce high-density lipoprotein (HDL) functionality. Thus, rescuing ApoA-I concentrations during inflammation might help to prevent atherosclerosis. Recent studies have shown that butyric acid (C4) has anti-inflammatory effects and rescues ApoA-I production. However, whether intestinal short chain fatty acids (SCFAs) are able to influence hepatic processes is unknown. Therefore, we investigated C4 anti-inflammatory effects on ApoA-I transcription in the intestineliver co-culture model. $\mathrm{C} 4$ dose-response experiments in the presence or absence of cytokines were performed in a co-culture system including Caco-2 cells, HepG2 cells, or both. Changes in ApoA-I transcription in Caco- 2 cells and HepG2 cells were analyzed using qPCR. C4 increased ApoA-I expression in HepG2 cells that cultured alone. When both cells were cultured together, C4 decreased ApoA-I expression in Caco-2 cells and increased ApoA-I expression in HepG2 cells. However, adding $\mathrm{C} 4$ to apical Caco-2 cells resulted in a smaller effect in HepG2 cells compared with adding $\mathrm{C} 4$ directly to the hepatocytes. Moreover, $\mathrm{C} 4$ rescued ApoA-I expression in inflamed HepG2 cells. These findings suggests that intestinal SCFAs can affect hepatic processes. However, the smaller effect in the co-culture experiment indicates crosstalk between intestine and liver. 


\section{Introduction}

A higher production of short chain fatty acids (SCFAs) in the intestinal lumen may result in various health benefits (1). Butyric acid (C4), the most studied SCFA, is mainly produced by colonic microbiota through the fermentation of water-soluble dietary fibers like pectin (2). In the past, it has been shown that $\mathrm{C} 4$ plays a local role in the physiology of the intestinal mucosa, since amongst other functions it is the major oxidative substrate for colonocytes (3). In addition, more recent studies have shown that $\mathrm{C} 4$ has local antiinflammatory effects in human colonic epithelial cells (4). In fact, C4 was reduced in intestinal biopsies of Crohn's disease patients' mRNA expression and production of pro-inflammatory cytokines, such as TNFa, by the inhibition of nuclear factor kappa $\mathrm{B}$ (NF- $\mathrm{kB}$ ) transactivation (5). Beside these local benefits, SCFAs also exert systemically beneficial effects, such as lowering inflammation in macrophages and endothelial cells through the inhibition of inflammatory cytokine production (6) and abating the development of atherosclerosis (7). These latter anti-atherosclerotic effects are thought to be mediated by modulating pro-inflammatory cytokine production, endothelial dysfunction and oxidative stress (8). Additionally, we have recently postulated that SCFAs may also affect atherosclerotic risk via elevating hepatic Apolipoprotein A-I (ApoA-I) transcription (9). To be effective systemically, SCFAs first need to be absorbed from the intestinal lumen by the enterocytes and transported into the circulation via the portal vein (10). This makes it tempting to suggest that SCFAs will most likely affect hepatic (patho)physiology, as shown, for example, in previous studies using liver (HepG2) cells $(9,11)$. In particular, the observation that $C 4$ could rescue the inflammation-induced reduction in ApoA-I transcription (12) is highly relevant, since ApoA-I is a negative acute phase protein, and its production is significantly lowered during inflammation (13). This consequent reduction in serum ApoA-I concentrations seems undesirable, especially during inflammation when the numerous beneficial effects of the ApoA-I protein itself $(14,15)$ are needed. However, in our earlier experiments mimicking hepatic inflammation, C4 was added directly to the medium of the HepG2 cells, while as mentioned earlier, SCFAs are formed in vivo in the intestinal lumen and need to be transported to the liver before any hepatic effects can be expected. However, various SCFAs do not reach the liver in similar concentrations since amounts produced and oxidation within the enterocytes might differ (16). To the best of our knowledge, the effects of butyrate on ApoA-I gene expression in the intestinal cells and the possible existence of cross-talk between enterocytes and hepatocytes on ApoA-I transcription are unknown. Theoretically, intraluminal-produced butyrate that is taken up by enterocytes might have a direct effect on hepatocytes when it reaches the liver (17). However, butyrate may also change the secretion of certain factors 
by intestinal cells (18), which affect the transcription of ApoA-I in liver cells. Therefore, to understand this in more detail, we here investigated changes in ApoA-I transcription after adding $\mathrm{C} 4$ to Caco-2 cells or HepG2 cells directly and compared these changes to the effects of adding $\mathrm{C} 4$ to the apical surface of Caco-2 cells, while analyzing ApoA-I expression in HepG2 cells cultured in the basolateral compartment in our co-culture model. These co-culture studies were performed in normal as well as under inflamed conditions.

\section{Material and methods}

\section{Materials}

Human epithelial colorectal adenocarcinoma (Caco-2) cells were obtained from ATCC (Molsheim, France). Human hepatocellular liver carcinoma cells (HepG2) were kindly provided by Sten Braesch-Andersen (Mabtech, Nacka Strand, Sweden). Cell culture flasks, plates and polyester membrane inserts ( $12 \mathrm{~mm}$ diameter, $0.4 \mu \mathrm{m}$ pore diameter) were obtained from Corning (Cambridge, MA, USA). Dulbecco's modified eagle medium (DMEM), Minimum essential medium (MEM), and sodium pyruvate and non-essential amino acids (NEAA) were all obtained from Thermo Fisher Scientific (Bleiswijk, Netherlands). Fetal bovine serum (FBS) was purchased from PAA (Toronto, Canada). Butyric acid (C4) was bought from Sigma (Uithoorn, Netherlands). The BET inhibitor JQ1(+) was obtained from Bio-Techne - R\&D (Minneapolis, MN, USA). Tumor necrosis factor-alpha (TNFa), interleukin-1beta (IL-1 $)$, dimethyl sulfoxide (DMSO), and Trireagent were all purchased from Sigma (Uithoorn, Netherlands).

\section{Cell culture and C4 treatment}

Caco- 2 cells and HepG2 cells were both cultured in T75 flasks (Corning, Cambridge, MA, USA) at $37^{\circ} \mathrm{C}$ in a humidified atmosphere of $5 \%$ carbon dioxide $\left(\mathrm{CO}_{2}\right)$ in medium (DMEM for Caco-2 and MEM for HepG2). Cell media contained 10\% heat inactivated FBS, $1 \%$ sodium pyruvate, $1 \%$ NEAA and $1 \%$ penicillin-streptomycin mixture. For all experiments, Caco- 2 cells were seeded to the inside of transwell inserts $(1.5 \mathrm{~mL} /$ well $)$ at a density of 300,000 cells $/ \mathrm{mL}$. For the first 21 days, the inserts were placed in 6-well plates. During these 21 days, the Caco- 2 cells were allowed to differentiate to their small intestinal phenotype (19). Next, the inserts were placed in new 6-well plates to determine whether the Caco-2 cell layers were confluent by examining phenol red leakage from the apical to the basolateral compartment (20). For this, MEM with phenol red was added to the apical compartment, while MEM without phenol red was added to the basolateral compartment for $48 \mathrm{~h}$ at $37^{\circ} \mathrm{C}$ 
in a humidified atmosphere of $5 \% \mathrm{CO}_{2}$. The optical density (OD) of the medium solution in both compartments was measured at $450 \mathrm{~nm}$ using a spectrophotometer (Beckman, Pasadena, CA, USA) (Figure 5.1). Only confluent Caco-2 monolayers (OD $450 \mathrm{~nm}$ of the basolateral medium less than 0.02) were used in the experiments. In addition, HepG2 cells were seeded in 6-well plates $(2.6 \mathrm{~mL} /$ well), at a density of $300,000 \mathrm{cells} / \mathrm{mL}$, and cultured for $48 \mathrm{~h}$. Three different conditions were used (Figure 5.2). First, Caco-2 cells were seeded in the inserts, without HepG2 cells in the lower compartment. Second, HepG2 cells were seeded in the lower compartment without Caco-2 cells on the insert above. Finally, Caco-2 cells were seeded on the insert in addition to HepG-2 cells in the lower compartment, and both of them were incubated together in a co-culture system to mimic the intestine-liver interaction. The three different setups of the transwell system were allowed to equilibrate for $48 \mathrm{~h}$ before the start of the experiments.

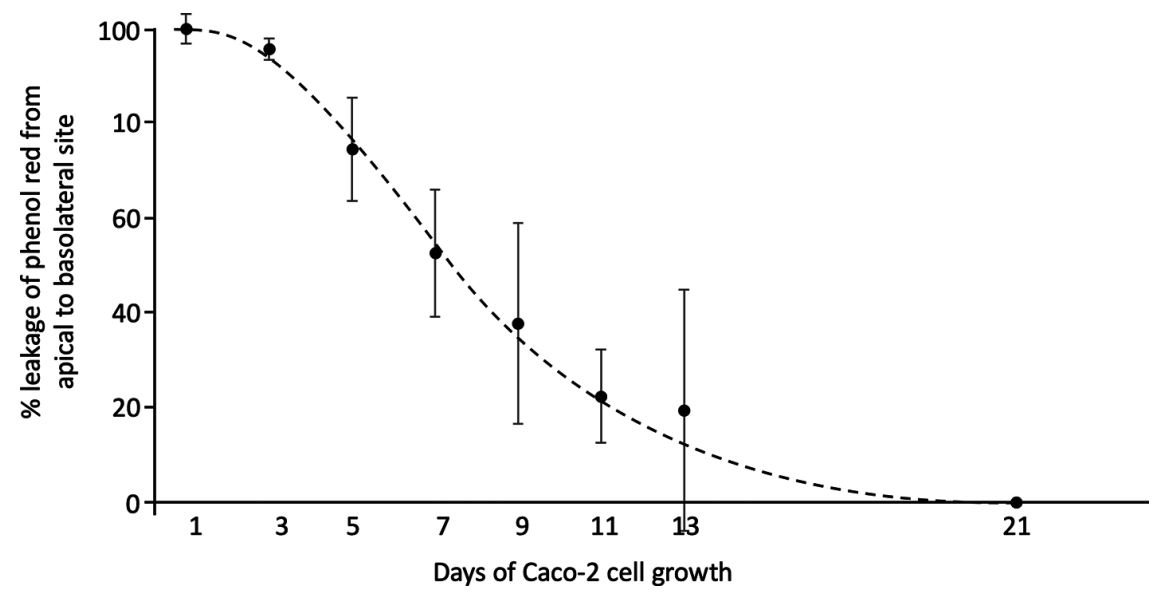

Figure 5.1. The percentage of phenol red leakage from the apical to the basolateral compartment in the transwell system. Medium solution (MEM) with phenol red was added to the apical compartment, while MEM without phenol red was added to the basolateral compartment. The optical density (OD) of the medium solution in both compartments was measured across the different days of Caco-2 cell growth. All results are presented as the mean, while error bars indicate standard deviations.

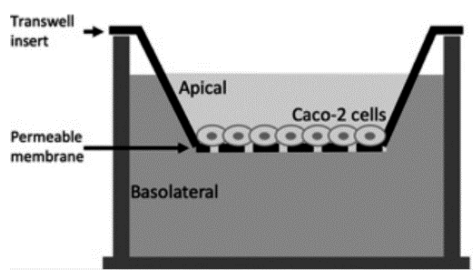

Condition 1

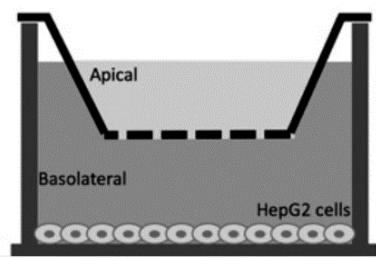

Condition 2



Condition 3

Figure 5.2. Schematic overview of different experimental conditions were performed in the transwell system. 
Several experiments were performed. In condition 1, C4 was added to the apical surface of Caco-2 cells in a concentration range of $0,1,2,4$, and $6 \mathrm{mM}$. In condition 2, C4 was added in the same concentration range to HepG2 cells. In condition 3, C4 was added to the apical surface of Caco-2 cells, again in the same concentration range, but this time HepG2 cells were present in the basolateral compartment. In this co-culture condition, we also evaluated the effect of an inflammatory component. For this, a cytokine cocktail of TNFa (with final concentration of $100 \mathrm{ng} / \mathrm{mL}$ ) and IL-1 $\beta$ (with final concentration of $5 \mathrm{ng} / \mathrm{mL}$ ) was added either only apically to the Caco- 2 cells, or only to HepG2 cells in the basolateral compartment, or simultaneously to both the apical and basolateral compartments. JQ1(+), a BET inhibitor, was included as a positive control in a separate well to ensure that cells were responsive to the treatments and produced sufficient amounts of ApoA-I mRNA (21). For this, JQ1(+) was added in final concentrations of $3 \mu \mathrm{M}$. Both C4 and JQ1(+) were dissolved in dimethyl sulfoxide (DMSO, cell culture tested), and effects were expressed relative to those of the carrier control (DMSO only). The final DMSO concentration was $0.2 \%$ in all samples. In all experiments, both Caco-2 and HepG2 cells were harvested for analysis of ApoA-I mRNA expression after lysing with Tri-reagent, as described (21) and stored at $-80{ }^{\circ} \mathrm{C}$ until further analysis.

\section{Quantification of gene mRNA transcription}

To evaluate the effects of C4 on ApoA-I mRNA expression, total RNA was isolated from the Caco-2 and HepG2 cells using Tri-reagent and the RNeasy mini kit (Qiagen, Hilden, Germany) according the manufacturer's instructions. For cDNA synthesis, 350 ng of total RNA was reverse transcribed using moloney murine leukemia virus (MMLV) reverse trans, dNTPs, random hexamers, dithiothreitol (DTT) and a 5xFS buffer supplemented with RNAse inhibitor (Thermo Fisher Scientific, Bleiswijk, Netherlands). The resulting cDNA was used for real time quantitative PCR using TaqMan gene expression assays using Cyclophilin A (Hs99999904) as a housekeeping control. To quantify ApoA-I, the TaqMan gene expression assays (Hs00163641) was used. Values are presented as relative gene expressions based on Ct values, normalized for the internal control Cyclophilin A and compared with control conditions.

\section{Statistical analysis}

All independent dose-response experiments with $\mathrm{C} 4$ were performed in duplicate, and each experiment was repeated three times. Six biological (12 technical) replicates were performed for every single $\mathrm{C} 4$ dose. Regression analysis was used to examine dose-response relationships between the concentration of added C4 and the mRNA expression of ApoA-I. 
For a dose-response relationship, the regression coefficients had to be significantly different from zero at $(\mathrm{p}<0.05)$. Effects of the positive control JQ1(+) in Caco-2 or HepG2 cells when cultured alone were statistically evaluated versus the control condition determined by a Mann-Whitney $U$ test, in which a p-value $<0.05$ was considered statistically significant. When evaluating the effects of JQ1(+) on ApoA-I mRNA expression in the different proinflammatory cytokines conditions in HepG2 or Caco-2 cells, the Mann-Whitney U test was used with a correction for multiple comparisons, in which a p-value $<0.008$ was considered statistically significant. In addition, when evaluating the effects of adding no cytokines versus cytokines added apical, basolateral, or to both compartments side-byside, the Mann-Whitney $U$ test was also used with a correction for multiple comparisons (requiring $\mathrm{p}<0.008$ for significance). All statistical analyses were performed using SPSS v.25 (IBM Corp., Armonk, NY, USA).

\section{Results}

\section{Effects of C4 on ApoA-I mRNA expression in Caco-2 or HepG2 cells (conditions 1 and 2)}

To evaluate the effects of $\mathrm{C} 4$ on Caco-2 cells (Condition 1, Figure 5.3A), different doses of $\mathrm{C} 4$ were added to the apical surface of the Caco- 2 cells on the insert, without HepG2 cells in the lower compartment. C4 did not change ApoA-I mRNA expression in the Caco-2 cells.

A

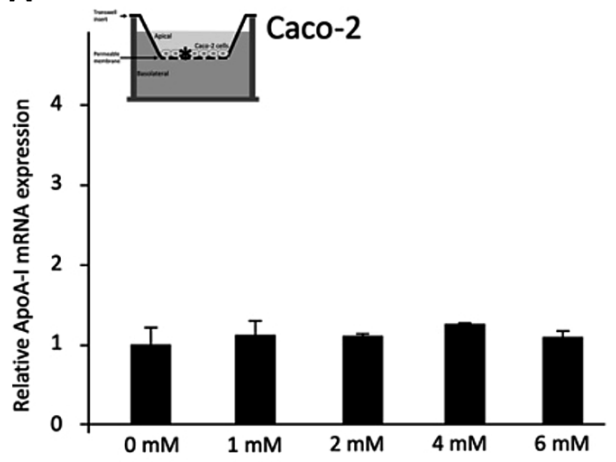

B

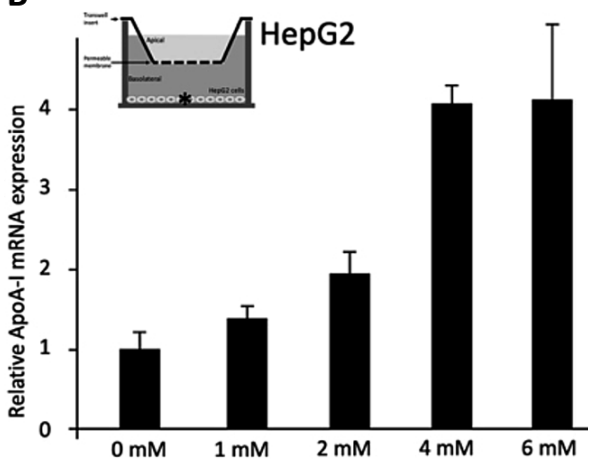

Figure 5.3. Relative Apolipoprotein A-I (ApoA-I) mRNA expressions in Caco-2 and HepG2 cells treated with different concentrations of C4. (A) Increasing C4 concentrations did not have any significant effects on ApoA-I mRNA expression in Caco-2 cells. (B) Increasing C4 concentrations showed a significant increase in ApoA-I mRNA expression in HepG2 cells $(\mathrm{p}<0.001)$. All results are presented as the mean, while error bars indicate standard deviations. Data were normalized against the expression observed in the control condition, which was arbitrarily set at 1 . A linear regression was performed for $\mathrm{C} 4$ dose-response effects. Changes were considered significant when the regression coefficients were significantly different from zero $(p<0.05)$. ApoA-I, apolipoprotein-I; mRNA, messenger RNA. 
When C4 was added to HepG2 cells in the lower compartment without the Caco-2 cells on the insert above (Condition 2, Figure 5.3B), ApoA-I mRNA expression dose-dependently increased $(\mathrm{p}<0.001)$, with a maximum of a 4.2 -fold at a $6 \mathrm{mM}$ concentration of $\mathrm{C} 4$.

\section{Effects of C4 added to the apical surface of Caco-2 cells on ApoA-I mRNA expres- sion in Caco-2 and HepG2 cells (condition 3)}

To explore the effects of $\mathrm{C} 4$ on Caco-2 and HepG2 cells co-cultured in a transwell system, C4 was added to the apical surface of Caco-2 cells cultured on inserts placed on top of the HepG2 cells in the lower compartment (Condition 3). In contrast to the condition without HepG2 cells (Figure 5.3A), ApoA-I gene expression in Caco-2 cells co-cultured with HepG2 cells significantly $(\mathrm{p}<0.01$ ) decreased after adding $\mathrm{C} 4$ (Figure 5.4A), whereas at the same time, ApoA-I mRNA expression in HepG2 cells was dose-dependently increased $(\mathrm{p}<0.001)$ (Figure 5.4B). When cytokines were added to the apical side of the Caco-2 cells, ApoA-I mRNA concentrations were significantly $(\mathrm{p}<0.008)$ lowered in both Caco-2 and HepG2 cells. Also, a significant decrease $(\mathrm{p}<0.008)$ was observed in both cell lines when cytokines were added to the basolateral compartment instead of the apical side. When cytokines were added to the apical side and basolateral compartment simultaneously, there was even a further reduction $(\mathrm{p}<0.008)$ in ApoA-I mRNA in both cell lines (Figure 5.4C-D). When C4 was added apically to the Caco-2 cells in the presence of cytokines (either apical, basolateral or both), C4 did not change ApoA-I mRNA expression in the Caco-2 cells (Figure 5.4C). In HepG2 cells, ApoA-I mRNA expression dose-dependently increased after adding C4 apically to the Caco-2 cells ( $\mathrm{p}<0.01$ ), also in the presence of cytokines either added apical, basolateral or in both compartments (Figure 5.4D). In other words, apical C4 was able to rescue the reduced ApoA-I mRNA expression in HepG2 cells exposed to pro-inflammatory cytokines but not in intestinal Caco- 2 cells.

\section{Effects of the positive control JQ1(+) on ApoA-I mRNA expression in Caco-2 and HepG2 cells}

The positive control JQ1(+) increased ApoA-I mRNA expression $(\mathrm{p}<0.05)$ in Caco-2 or HepG2 cells when cultured alone (Figure 5.5). As mentioned before and shown in Figures 5.4C and 5.4D, ApoA-I mRNA expression in cells exposed to pro-inflammatory cytokines was lower compared with mRNA expression in non-inflamed cells. Effects of JQ1(+) treatment in these inflammatory conditions are shown in Figure 5.5. JQ1(+) did not affect ApoA-I gene expression in Caco-2 cells (co-cultured with HepG2 cells) without cytokines or when cytokines were added to either apical, basolateral or both compartments. Moreover, JQ1(+) significantly ( $\mathrm{p}$ < 0.008) increased ApoA-I gene expression in HepG2 cells (co- 
A

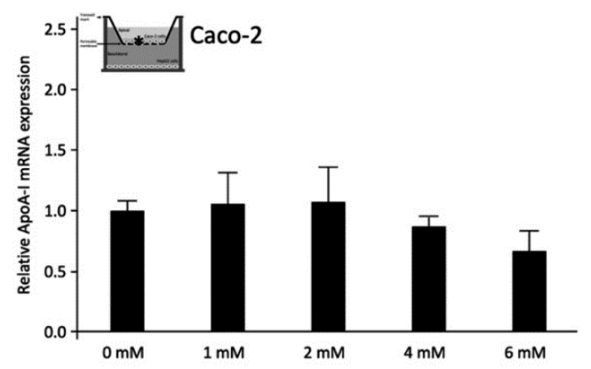

C

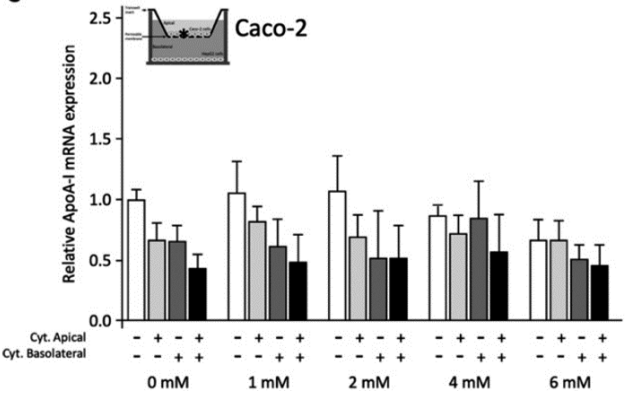

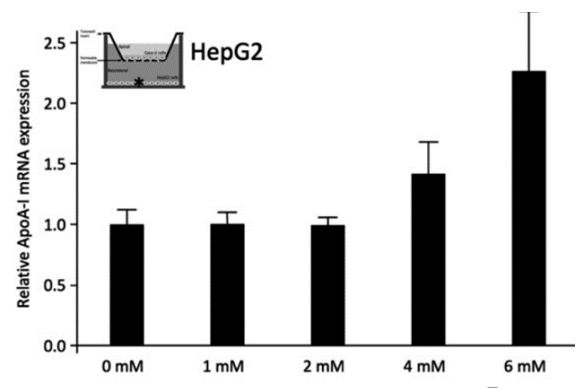

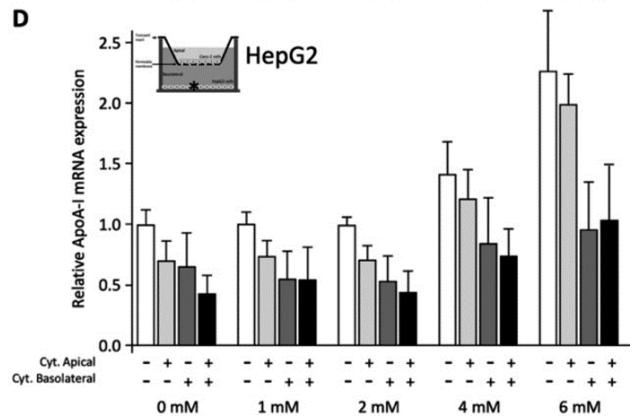

Figure 5.4. Relative Apolipoprotein A-I (ApoA-I) mRNA expressions in Caco-2 and HepG2 cells were cultured in a transwell system and treated with different concentrations of $\mathrm{C} 4$. All results are presented as the mean, while error bars indicate standard deviations. Data were normalized against the expression observed in the control condition, which was arbitrarily set at 1 . A linear regression was performed for $\mathrm{C} 4$ dose-response effects. Changes were considered significant when the regression coefficients were significantly different from zero $(\mathrm{p}<0.05 ; \mathbf{A}-\mathbf{D})$. When evaluating the effects of adding no cytokines versus cytokines added apical, basolateral or to both compartments side-by-side, the Mann-Whitney $U$ test was used with a correction for multiple comparisons in which a p-value $<0.008$ was considered statistically significant (C-D). (A) Increasing C4 concentrations showed a significant reduction in ApoA-I mRNA expression in Caco-2 cells that were cultured alone $(\mathrm{p}<0.01)$. (B) Increasing C4 concentrations showed a significant increase in ApoA-I mRNA expression in HepG2 cells that were cultured alone $(p<0.001)$. (C) Increasing C4 concentrations did not show any significant effects on ApoA-I mRNA expression in Caco-2 cells co-cultured with HepG2 cells in the presence of cytokines (either apical, basolateral or both compartments). (D) Increasing C4 concentrations showed a significant increase in ApoA-I mRNA expression in HepG2 cells co-cultured with Caco-2 cells in the presence of cytokines (either apical, basolateral or both compartments) $(p<0.01)$. When cytokines were added to the apical side of the Caco-2 cells, ApoA-I mRNA expression significantly $(\mathrm{p}<0.008)$ decreased in both Caco-2 and HepG2 cells (C-D). A significant reduction in ApoA-I mRNA expression ( $<<0.008)$ was observed in Caco-2 and HepG2 cells when cytokines were added to the basolateral compartment instead of the apical side. When the cytokines were added to both the apical and basolateral compartments, there was an even further reduction $(\mathrm{p}<0.008)$ in ApoA-I mRNA expression in Caco-2 and HepG2 cells. The presence of cytokines was indicated with (+), while the absence of cytokines was indicated with (-). ApoA-I, apolipoprotein-I; mRNA, messenger RNA.

cultured with Caco-2 cells) with or without cytokines added to the apical compartment. Furthermore, JQ1(+) did not affect ApoA-I gene expression in HepG2 cells (co-cultured with Caco-2 cells) with cytokines added basolaterally or to both compartments (Figure 5.5). Taken together, the cells responded to JQ1(+) as expected (21). 


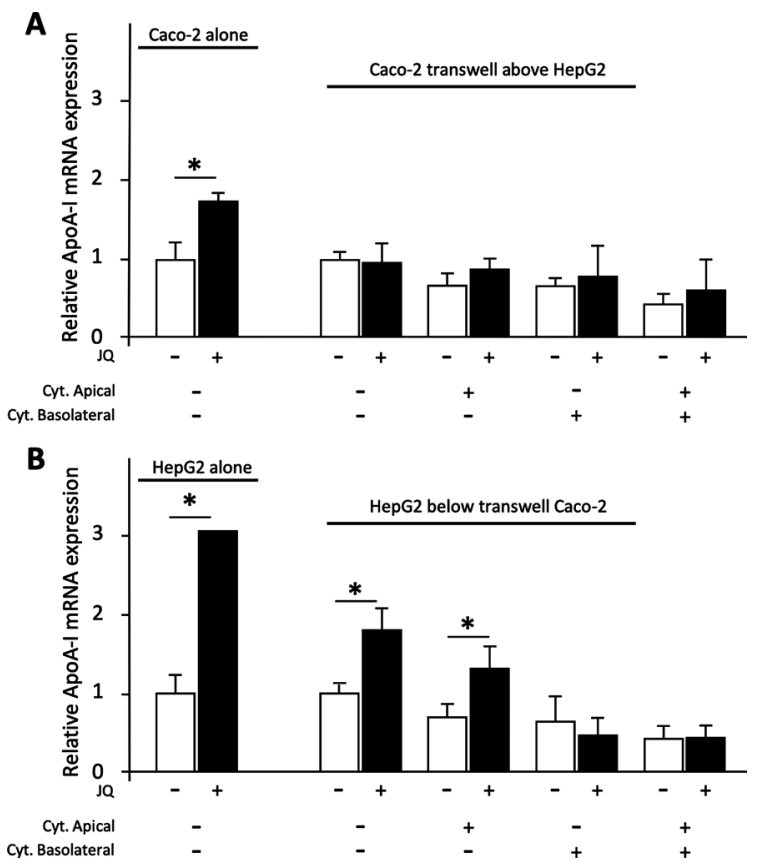

Figure 5.5. Relative Apolipoprotein A-I (ApoA-I) mRNA expressions in Caco-2 and HepG2 cells were cultured in a transwell system and treated with the positive control JQ1(+) $(3 \mu \mathrm{M})$. (A) JQ1(+) significantly increased ApoA-I mRNA expression in Caco-2 cells that were cultured alone $(\mathrm{p}<0.05)$. JQ1(+) did not affect ApoA-I gene expression in Caco-2 cells co-cultured with HepG2 cells without cytokines or when cytokines were added to either apical, basolateral or both compartments. (B) JQ1(+) significantly increased ApoA-I mRNA expression in HepG2 cells that were cultured alone $(\mathrm{p}<0.01)$. JQ1 $(+)$ significantly increased ApoA-I mRNA expression in HepG2 cells co-cultured with Caco-2 cells without cytokines or when cytokines were added to apical compartments $(\mathrm{p}<0.008)$. JQ1 $(+)$ did not affect ApoA-I gene expression in HepG2 cells co-cultured with Caco-2 cells when cytokines were added basolaterally or to both compartments. All results are presented as the mean, while error bars indicate standard deviations. Data were normalized against the expression observed in the control condition, which was arbitrarily set at 1 . A Mann-Whitney U test was performed to evaluate JQ1(+) effects in Caco-2 or HepG2 cells that were cultured alone versus control conditions, in which a p-value $<0.05$ was considered statistically significant. When evaluating the effects of JQ1(+) on ApoA-I mRNA expression in different pro-inflammatory cytokines conditions in HepG2 or Caco2 cells, the Mann-Whitney $\mathrm{U}$ test was used with a correction for multiple comparisons, in which a p-value $<0.008$ was considered statistically significant. Changes are indicated with ${ }^{\star}$ when the effect of JQ1(+) is significantly different from the control. The presence of cytokines was indicated with $(+)$, while the absence of cytokines was indicated with (-). ApoA-I, apolipoprotein-I; mRNA, messenger RNA.

\section{Discussion}

In earlier experiments, we have shown that C4 treatment increased ApoA-I mRNA expression in HepG2 cells in both normal and inflammatory conditions $(9,12)$. These positive effects on hepatic ApoA-I transcription were observed when $\mathrm{C} 4$ was added directly to HepG2 cells. Here, we have evaluated whether these effects were still evident when C4 was added to the apical surface of enterocytes, which means that it first had to be taken 
up by Caco 2 cells and transported towards HepG2 cells in the basolateral compartment. This also made it possible to evaluate the effects of $\mathrm{C} 4$ on ApoA-I expression in Caco 2 cells, which also contribute to ApoA-I concentrations in the circulation (22). Although C4 is mainly produced by microbiome in the colon, we decided to examine the effects of $\mathrm{C} 4$ on ApoA-I transcription in small intestinal enterocytes since ApoA-I is mainly produced in the duodenum and jejunum $(23,24)$. However, although SCFAs as end products of bacterial fermentation are present in higher concentrations in the colon, SCFAs are also present in the proximal intestines $(23,25)$. We did not find any effect of C4 on ApoA-I gene expression in Caco2 cells when cultured alone (condition 1). In contrast to the effects in Caco-2 cells, but in line with our earlier findings (9), C4 again significantly increased ApoA-I expression in HepG2 cells (condition 2). Interestingly, in the co-culture model (condition 3), we found that ApoA-I mRNA expression in Caco-2 cells was even lowered after adding C4 in the presence of HepG2 cells, whereas effects of $\mathrm{C} 4$ on HepG2 cells remained positive, showing a significant increase in ApoA-I gene expression. This illustrates the cell-specific response of enterocytes and hepatocytes in a co-culture model in response to $\mathrm{C} 4$ exposure. Moreover, in the presence of cytokines either added to apical, basolateral or to both cell compartments, ApoA-I mRNA levels in HepG2 cells were rescued by C4. However, the effects of C4 on elevating ApoA-I mRNA expression in HepG2 cells when added to the apical side of Caco2 cells in the transwell were lower compared with the effects of $\mathrm{C} 4$ when added directly to HepG2 cells. The question is how the lower hepatic effects of $\mathrm{C} 4$ in the co-culture experiments can be explained. Theoretically, decreased bioavailability (i.e., the amount of SCFAs that are transported from the apical to the basolateral side), which translates into lower hepatic exposure to C4, seems the most logical explanation. It is well-known that SCFAs are used as fuel by the intestinal cells (26). Therefore, once SCFAs are metabolized by the intestinal cells, they will not be available (in their original concentrations) in the basolateral compartment, hence explaining the lower bioavailability. Moreover, we also observed that the inhibitory effect on hepatic ApoA-I expression was larger when cytokines were added directly to HepG2 cells. This finding, in the case of cytokines, could also attributed to the bioavailability of cytokines. However, in contrast to $\mathrm{C} 4$, cytokines are not used for fueling enterocytes, which again raises the question of why cytokines' effects on HepG2 cells were smaller when they were added apically in the transwell system. An alternative explanation for the lower effects of both C4 and cytokines on HepG2 cells after adding them to Caco- 2 cells could also theoretically be a cross-talk phenomenon between Caco- 2 cells and HepG2 cells. For example, the secreted factors from the Caco- 2 cells into the basolateral compartment might influence the effects of C4 on HepG2 cells. In theory, such cross-talk between intestine and liver may be critical for human health since several liver diseases result from alterations in the intestinal barrier (27). Moreover, such interactions between the intestine, including 
its microbiota, and the liver can also be regulated by exposure to dietary compounds (27). A recent study investigated the effects of SCFAs on the gut-liver axis in interconnected human micro-physiological systems (MPS) (28). Adding SCFAs to the apical enterocyte side favorably modulated the gut-liver axis in MPS by innate immune inactivation. Moreover, Trapecar et al. also showed, by using the same MSP system, that apical addition of SCFAs to enterocytes not only inhibited gut inflammation but also increased liver metabolic function, improved lipid metabolism and enhanced hepatic bile acid secretion (28). Altogether, this illustrates that hepatic effects exist after intestinal exposure to SCFAs. However, this can still be simple response to SCFAs transferred from the intestine to the liver, or cross-talk of the liver responding to factors secreted via intestinal cells. To the best of our knowledge, there is so far no known intestinal factor that is secreted in response to SCFAs exposure, influencing hepatic physiology. However, our data may suggest the existence of such a factor. We showed that Caco- 2 cells responded in the opposite direction to C4 compared with HepG2 cells. To elaborate, treating Caco-2 cells with $\mathrm{C} 4$ had no effects on ApoA-I mRNA when the Caco-2 cells were cultured alone and even decreased intestinal ApoA-I mRNA expression in the co-culture model. The question now is whether we should expect effects on the intestine and liver to go hand-in-hand or whether there are other known conditions where effects on the liver and intestine were also in the opposite direction. A potential difference in ApoA-I mRNA response between the intestinal and hepatic cells could be explained by the need of some coactivators to produce ApoA-I, which is absent in Caco-2 cells. For example, the synergy between the ApoA-I promoter and the ApoCIII enhancer is essential to induce intestinal ApoA-I transcription, whereas the induction of hepatic ApoA-I gene expression seems independent of the ApoCIII enhancer (29). However, although the absence of such coactivators might explain why $\mathrm{C} 4$ had no effects on Caco-2 cells when cultured alone, we have now even observed inhibition of ApoA-I expression in Caco-2 in the co-culture model after $\mathrm{C} 4$ exposure. This suggests that effects of $\mathrm{C} 4$ on Caco- 2 cells might activate different pathways that not only translate into lower ApoA-I transcription but could also induce the production of a currently unknown factor. These factors might not only affect ApoA-I transcription in the enterocyte itself but also influence hepatic function, which is evident in our studies as a lower hepatic ApoA-I transcription. Identification of such a factor would be highly informative and deserves further studies. Finally, although we acknowledge that the various SCFAs have different systemic concentrations, we expect other SCFAs to behave identically (1). On the other hand, as explained, it could be that there are differences regarding intestinal cells metabolism between different SCFAs, which might consequently result in different basolateral concentrations and different effects on liver cells. However, since the concentrations of acetate and propionate in vivo are likely to be higher than those of butyrate, it seems "safe" to speculate that comparable effects will be observed for other 
SCFAs.

In conclusion, our findings indicate that in co-culture experiments, adding $\mathrm{C} 4$ apically to intestinal cells does not increase the lower ApoA-I mRNA level in inflamed Caco-2 cells. Furthermore, C4 added apically to Caco-2 cells elevates hepatic ApoA-I transcription and rescues the lower ApoA-I expression in inflamed HepG2 cells. As we found previously that all SCFAs (in line with C4) were able to enhance hepatic ApoA-I expression when directly added to HepG2 cells (9), we suggest further exploration of the effects of other SCFAs in the intestine-liver co-culture model. Moreover, the effects of adding C4 to the apical side of enterocytes translate into a smaller effect on HepG2 cells compared with adding C4 to hepatocytes directly. We speculate that this could be due to lower bioavailability, but it could also indicate cross-talk between intestine and liver. Identification of such an enterocytederived inhibitory factor warrants further studies.

\section{References}

1. Kles, K.A.; Chang, E.B. Short-chain fatty acids impact on intestinal adaptation, inflammation, carcinoma, and failure. Gastroenterology. 2006, 2, S100-S105.

2. Waldecker, M.; Kautenburger, T.; Daumann, H.; Veeriah, S.; Will, F.; Dietrich, H. Histone-deacetylase inhibition and butyrate formation: Fecal slurry incubations with apple pectin and apple juice extracts. Nutrition. 2008, 4, 366-374.

3. Robles-Vera, I.; Toral, M.; de la Visitación, N.; Aguilera-Sánchez, N.; Redondo, J.M.; Duarte, J. Protective Effects of Short-Chain Fatty Acids on Endothelial Dysfunction Induced by Angiotensin II. Front. Physiol. 2020, 11, 277.

4. Canani, R.B.; Di Costanzo, M.; Leone, L.; Pedata, M.; Meli, R.; Calignano, A. Potential beneficial effects of butyrate in intestinal and extraintestinal diseases. World J. Gastroenterol. 2011, 12, 1519.

5. Segain, J.; De La Blétiere, D.R.; Bourreille, A.; Leray, V.; Gervois, N.; Rosales, C. Butyrate inhibits inflammatory responses through NFkB inhibition: Implications for Crohn's disease. Gut. 2000, 3, 397-403.

6. Li, M.; van Esch, B.C.; Wagenaar, G.T.; Garssen, J.; Folkerts, G.; Henricks, P.A. Pro-and anti-inflammatory effects of short chain fatty acids on immune and endothelial cells. Eur. J. Pharmacol. 2018, 11, 52-59.

7. Ohira, H.; Tsutsui, W.; Fujioka, Y. Are short chain fatty acids in gut microbiota defensive players for inflammation and atherosclerosis? J. Atheroscler. Thromb. 2017, 7, 660-672.

8. Thibault, R.; Blachier, F.; Darcy-Vrillon, B.; De Coppet, P.; Bourreille, A.; Segain, J-P. Butyrate utilization by the colonic mucosa in inflammatory bowel diseases: A transport deficiency. Inflammatory bowel diseases. Inflamm. Bowel Dis. 2010, 4, 684-695.

9. Tayyeb, J.Z.; Popeijus, H.E.; Mensink, R.P.; Konings, M.C.; Mulders, K.H.; Plat, J. The effects of short-chain fatty acids on the transcription and secretion of apolipoprotein A-I in human hepatocytes in vitro. J. Cell. Biochem. 2019, 10, 17219-17227. 
10. Bloemen, J.G.; Venema, K.; van de Poll, M.C.; Damink, S.W.O.; Buurman, W.A.; Dejong, C.H. Short chain fatty acids exchange across the gut and liver in humans measured at surgery. Clin. Nutr. 2009, 6, 660-672.

11. Den Besten, G. Elucidating the Mechanisms of Action of Short-Chain Fatty Acids: From Dietary Fiber to Host Metabolism. Ph.D. Thesis, Groningen University, Groningen, The Netherlands, 2014; ISBN 978-90-367-6877-1.

12. Tayyeb, J.Z.; Popeijus, H.E.; Mensink, R.P.; Konings, M.; Mokhtar, F.B.A.; Plat, J. Short-Chain Fatty Acids (Except Hexanoic Acid) Lower NF-kB Transactivation, Which Rescues Inflammation-Induced Decreased Apolipoprotein A-I Transcription in HepG2 Cells. Int. J. Mol. Sci. 2020, 14, 5088.

13. Carpintero, R.; Pineiro, M.; Andres, M.; Iturralde, M.; Alava, M.; Heegaard, P.M. The concentration of apolipoprotein AI decreases during experimentally induced acute-phase processes in pigs. Infect. Immun. 2005, 5, 3184-3187.

14. Miyazaki, A.; Sakuma, S.; Morikawa, W.; Takiue, T.; Miake, F.; Terano, T. Intravenous injection of rabbit apolipoprotein AI inhibits the progression of atherosclerosis in cholesterol-fed rabbits. Arterioscler. Thromb. Vasc. Biol. 1995, 11, 1882-1888.

15. Shah, P.K.; Yano, J.; Reyes, O.; Chyu, K.-Y.; Kaul, S.; Bisgaier, C.L. High-Dose Recombinant Apolipoprotein A-IMilano Mobilizes Tissue Cholesterol and Rapidly Reduces Plaque Lipid and Macrophage Content in Apolipoprotein E-Deficient Mice: Potential Implications for Acute Plaque Stabilization. Circulation. 2001, 25, 3047-3050.

16. Cummings, J.; Pomare, E.; Branch, W.; Naylor, C.; Macfarlane, G. Short chain fatty acids in human large intestine, portal, hepatic and venous blood. Gut. 1987, 10, 1221-1227.

17. Schmitt Jr, M.G.; Soergel, K.H.; Wood, C.M. Absorption of short chain fatty acids from the human jejunum. Gastroenterology. 1976, 2, 211-215.

18. Lammi, C.; Zanoni, C.; Ferruzza, S.; Ranaldi, G.; Sambuy, Y.; Arnoldi, A. Hypocholesterolaemic activity of lupin peptides: Investigation on the crosstalk between human enterocytes and hepatocytes using a co-culture system including Caco-2 and HepG2 cells. Nutrients. 2016, 7, 437.

19. Van Breemen, R.B.; Li, Y. Caco-2 cell permeability assays to measure drug absorption. Expert Opin. Drug Metab. Toxicol. 2005, 2, 175-185.

20. Garcia, M.N.; Flowers, C.; Cook, JD. The Caco-2 cell culture system can be used as a model to study food iron availability. J. Nutr. 1996, 1, 251-258.

21. Van der Krieken, S.E.; Popeijus, H.E.; Mensink, R.P.; Plat, J. Link between ER-stress, PPAR-alpha activation, and BET inhibition in relation to apolipoprotein A-I transcription in HepG2 cells. J. Cell. Biochem. 2017, 8, 2161-2167.

22. Wu, A.L.; Windmueller, H.G. Relative contributions by liver and intestine to individual plasma apolipoproteins in the rat. J. Biol. Chem. 1979, 15, 7316-7322.

23. Peng, L.; He, Z.; Chen, W.; Holzman, I.R.; Lin, J. Effects of butyrate on intestinal barrier function in a Caco-2 cell monolayer model of intestinal barrier. Pediatrics Res. 2007, 1, 37-41.

24. Glickman, R.M.; Green, P.H. The intestine as a source of apolipoprotein A1. Proc. Natl. Acad. Sci. USA 1977, 6, 2569-2573.

25. Neis, E.P.; van Eijk, H.M.; Lenaerts, K.; Damink, S.W.O.; Blaak, E.E.; Dejong, C.H. Distal versus proximal intestinal short-chain fatty acid release in man. Gut. 2019, 4, 764-765.

26. Parada Venegas, D.; De la Fuente, M.K.; Landskron, G.; González, M.J.; Quera, R.; Dijkstra, G. Short Chain Fatty Acids (SCFAs)-mediated gut epithelial and immune regulation and its relevance for inflammatory bowel diseases. Front. Immunol. 2019, 10, 277. 
27. Albillos, A.; de Gottardi, A.; Rescigno, M. The gut-liver axis in liver disease: Pathophysiological basis for therapy. J. Hepatol. 2020, 3, 558-577.

28. Trapecar, M.; Communal, C.; Velazquez, J.; Maass, C.A.; Huang, Y.-J.; Schneider, K. Gut-Liver physiomimetics reveal paradoxical modulation of IBD-related inflammation by short-chain fatty acids. Cell Syst. 2020, 3, 223-239.

29. Kan, H.-Y.; Georgopoulos, S.; Zannis, V. A hormone response element in the human apolipoprotein CIII (ApoCIII) enhancer is essential for intestinal expression of the ApoA-I and ApoCIII genes and contributes to the hepatic expression of the two linked genes in transgenic mice. J. Biol. Chem. 2000, 39, 30423-30431. 


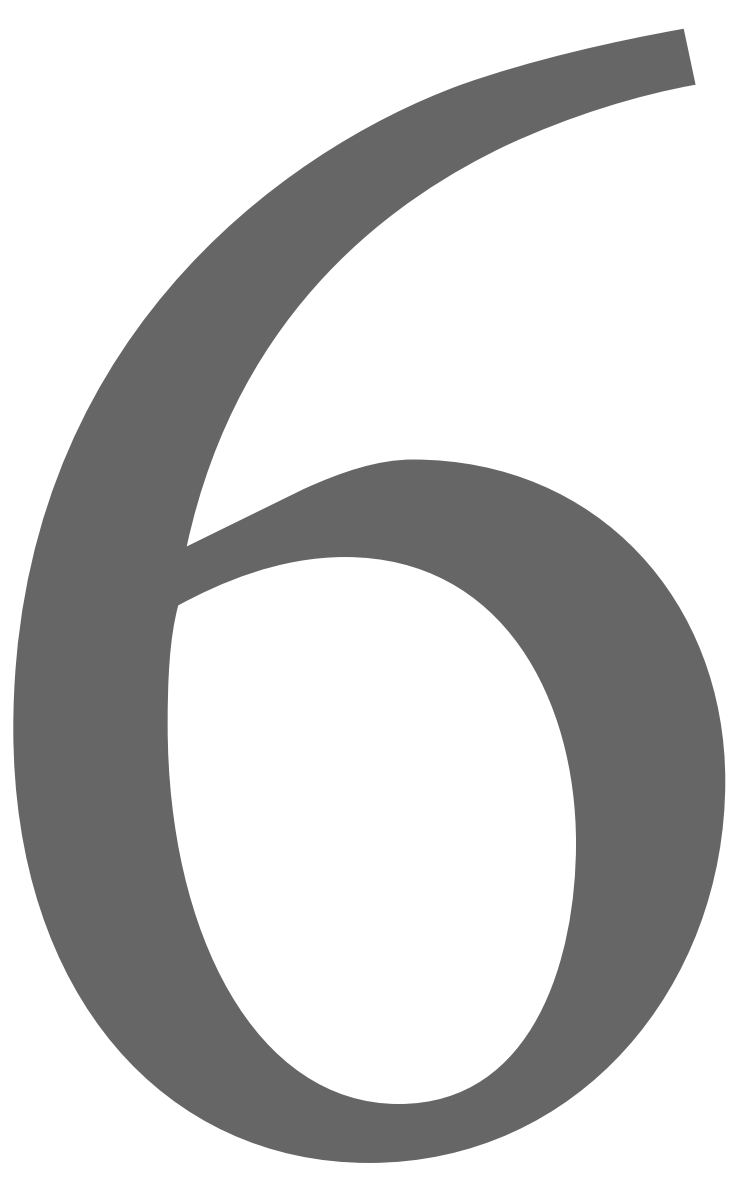




\section{Differential effects of individual amino acids on PPARa transactivation, ApoA-I transcription and (pro)-ApoA-I secretion}

Jehad Z. Tayyeb /Herman E. Popeijus | Ronald P. Mensink | Jogchum Plat 


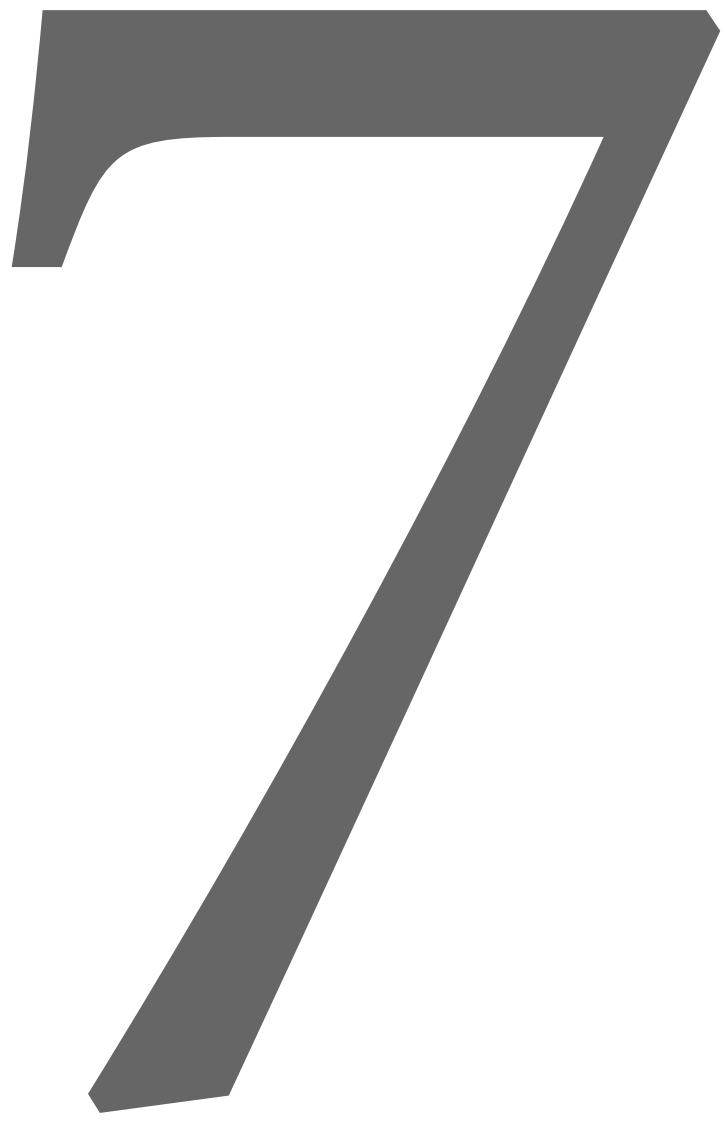


General discussion 


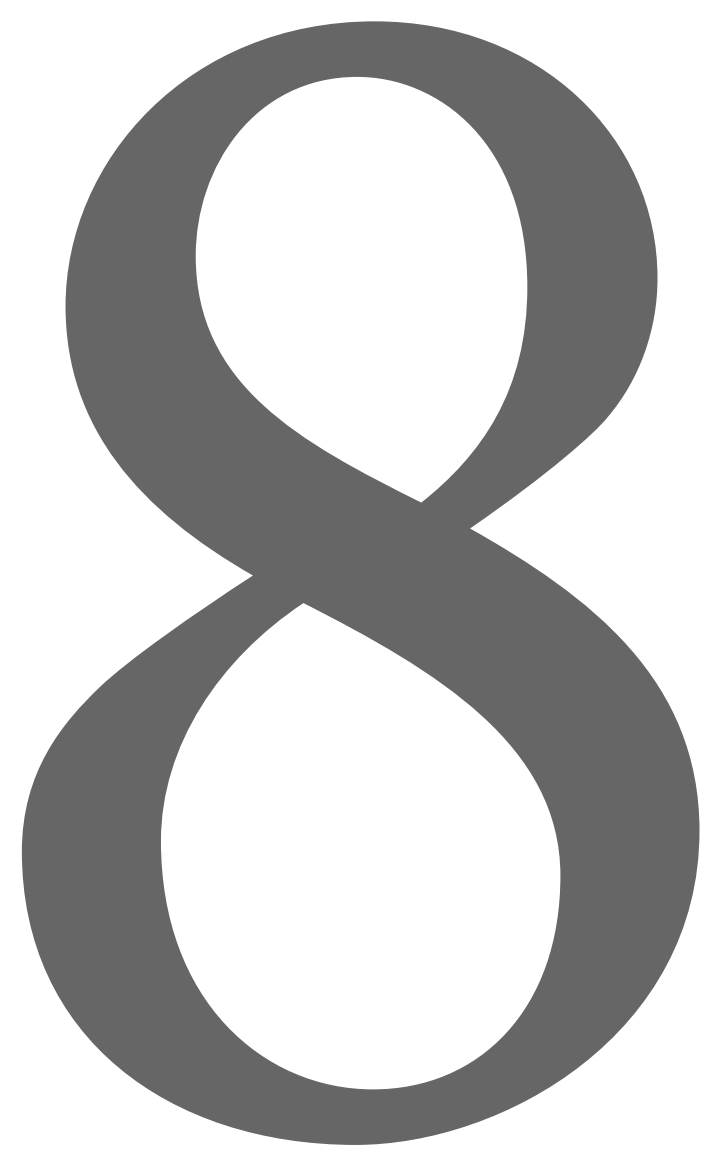


Summary 



\section{Summary}

Cardiovascular diseases (CVD) are the leading cause of death in the western countries. Dyslipidemia, one of the modifiable CVD risk factors, is defined as having high plasma low-density lipoprotein (LDL) cholesterol and triacylglycerol concentrations or low levels of serum high-density lipoprotein (HDL) cholesterol. Indeed, several epidemiological studies have shown an inverse relation between HDL cholesterol levels and CVD risk. Surprisingly, recent clinical studies however showed that increasing serum HDL cholesterol concentrations by pharmacological interventions failed to reduce CVD risk. Thus, the focus has switched to improving HDL functionality instead of elevating serum HDL cholesterol concentrations. Increasing evidence suggests that HDL functionality plays a major role in inflammation and atherogenesis. For example, HDL particles promote reverse cholesterol transport (RCT), a mechanism by which accumulated cholesterol is transported from the arterial walls to the liver for excretion, which consequently reduces atherosclerosis development. Apolipoprotein A-I (ApoA-I), the major protein component of HDL particles, has been identified as an important player in explaining HDL functionality and RCT. Therefore, approaches that target to elevate ApoA-I is a promising strategy to reduce CVD risk. In this thesis we therefore focused on the effects of antibiotics, short-chain fatty acids (SCFAs) and amino acids on ApoA-I transcription. In addition, we evaluated the effects of SCFAs on ApoA-I expression in a co-culture model by culturing enterocytes and hepatocytes together in a transwell system.

In an earlier clinal trial, the plasma HDL cholesterol concentration was significantly reduced after antibiotic (amoxicillin) treatment. This finding raised the question whether this HDL cholesterol lowering effect by amoxicillin was a direct effect on lipoprotein metabolism or an indirect effect resulting from alterations in the composition of microbiota and consequent SCFAs concentrations. To investigate the potential direct effect, we examined the effects on ApoA-I transcription when different concentrations of various antibiotics were added to the HepG2 and Caco-2 cells (chapter 3). In line with the clinal trial, we found that amoxicillin significantly reduced both ApoA-I mRNA expression and protein secretion in HepG2 and Caco-2 cells. We also examined potential mechanisms underlying these direct effects of antibiotic on ApoA-I expression. We found that amoxicillin treatment inhibited PPAR $a$ transactivation, which was confirmed by a lowered mRNA expression of CPT1, a well-known PPARa target gene. Therefore, we concluded that antibiotic treatment can directly affects lipoprotein metabolism via inhibiting ApoA-I transcription and secretion. In chapter 2, we examined the potential indirect effect of the antibiotics on HDL metabolism by evaluating dose-response relationships between the different SCFAs with ApoA-I transcription. Interestingly, increasing SCFAs concentrations significantly increased ApoA-I 
mRNA expression in HepG2 cells. This increase was associated with the enhanced PPARa transactivation, which indicates that the PPARa pathway is most likely involved in the positive effects of SCFAs on ApoA-I mRNA expression. These findings further suggest that the effects on serum HDL cholesterol concentrations as observed in our human intervention study with amoxicillin could be due to a combination of direct inhibitory effects on ApoA-I transcription as well as indirect effects via reduced SCFAs production. Moreover, previous studies have suggested that ApoA-I concentrations are reduced during inflammation, since ApoA-I is a negative acute phase protein. To evaluate effects of SCFAs on ApoA-I expression in the inflammatory condition, we performed our SCFAs experiments also in inflamed HepG2 cells (chapter 4). In line with the normal condition, SCFAs also exerted their beneficial effects on ApoA-I during inflammation. Moreover, SCFAs were able to recover the reduced level of ApoA-I expression in the inflamed HepG2 cells up the normal level, and even increased ApoA-I expression at higher concentrations. In addition, SCFAs treatment inhibited NF- $\kappa$ B transactivation and lowered IL-8 secretion from the inflamed HepG2 cells. Our data suggest that PPAR $a$ transactivation also underlies these anti-inflammatory effects of SCFAs in the inflamed HepG2 cells. Although SCFAs are produced mainly by microbiota in the colon, they are also produced in lower amounts in the small intestine. It is known that the intraluminally produced SCFAs are taken up by the intestinal cells and used as energy source. However, a part of the SCFAs is transported to the liver via the portal vein. In addition, their uptake by enterocytes might trigger the secretion of factors that could be transported from the enterocytes to the liver and affect hepatic processes. To study such a possible cross-talk between intestine and liver on hepatic ApoA-I expression, we evaluated the effect of $\mathrm{C} 4$ on hepatic ApoA-I mRNA expression in a co-culture system including Caco2 and HepG2 cells (chapter 5). The positive effects of SCFAs on hepatic ApoA-I expression were also observed in these co-culture experiments after adding $\mathrm{C} 4$ apically to the intestinal cells. However, the effect of $\mathrm{C} 4$ on hepatic ApoA-I expression in this study was smaller than the effect of $\mathrm{C} 4$ when it was added directly to the liver cells. This latter finding indeed suggests a lower bioavailability of C4. Also, it might be the result from a cross-talk between the intestine and liver cells. In that situation the enterocytes should secrete an inhibitory factor to dampen hepatic ApoA-I expression. Finally, since some studies have suggested that amino acids influence PPAR $a$ transactivation, we explored in chapter 6 the effects of different amino acids on ApoA-I expression in HepG2 cells. Interestingly, leucine, glutamic acid and tryptophan increased mRNA expressions of both ApoA-I and PPARa. Therefore, amino acids could be interesting targets for further studies to increase ApoA-I production.

In summary, the research in this thesis was performed to get more insights into the effects of external factors like antibiotics, SCFAs and amino acids on hepatic and intestinal ApoA-I 
transcription. Besides the negative effect of antibiotic on ApoA-I transcription and secretion, our findings have shown that SCFAs have positive effects on ApoA-I expression in HepG2 cells in both normal and inflammatory conditions. Moreover, $\mathrm{C} 4$ treatment also increased hepatic ApoA-I mRNA expression in the intestine-liver co-culture model. Finally, different amino acids were identified as natural compounds that have the potency to transactivate PPAR $\alpha$ and consequently increased hepatic ApoA-I expression. 







\section{Impact paragraph}





\section{Social and economic relevance}

In this section, the potential impact of our scientific findings for society and economy will be described. This mostly concerns the possible future applications of our findings in specific target groups, i.e. subjects with risk factors for cardiovascular diseases (CVD) such as dyslipidemia. Despite the fact that treatment has improved tremendously over the past decades, CVD remains the leading cause of morbidity and mortality worldwide (1). The most recent world health organization (WHO) report indicates that more than 17.9 million persons die from CVD each year. Currently, the number of newly diagnosed CVD cases in Europe is more than 11 million yearly, which results in a high economic burden and concomitant health care related costs of around $€ 210$ billion per year (1). Clearly, there is a need to discover and use additional therapies on top of already existing portfolios to further reduce the risk of CVD development and the high costs of CVD-related medical care. Although some studies showed conflicting results about the link between elevating circulating high-density lipoprotein (HDL) cholesterol concentrations and CVD (2), recent experimental studies have shown that CVD can be reduced by the enhancement of HDL functionality (3). The main function of HDL is to transport the excess cholesterol from peripheral tissues to the liver for excretion, a process called a reverse cholesterol transport (RCT) (4). Recent evidence suggested that increasing RCT process protects against CVD and atherosclerosis (5). Therefore, targeting factors known to stimulate RCT, such as increasing apolipoprotein A-I (ApoA-I) synthesis (6), is a promising strategy to reduce the progression of atherosclerosis development and consequent CVD. Although there are already some pharmaceutical options to increase ApoA-I production in specific high risk patient populations (7), dietary recommendations that aim to increase ApoA-I and HDL functionality may be needed to prevent CVD. Therefore, in our studies we aimed to discover the potential contribution of some (dietary natural) compounds on ApoA-I production with the ultimate aim to lower CVD risk and progression, and the financial costs of current CVD treatment. Thus, the main goal of this thesis was to explore the effects of several external stimuli on ApoA-I transcription and secretion. We focused on the effects of SCFAs, antibiotics and amino acids to understand underlying mechanisms and to generate leads for future CVD treatment and prevention.

\section{Scientific gain of this thesis}

The research in this thesis supported our previous observation from a placebo-controlled double blind human intervention trial showing that antibiotic treatment was linked to alterations in metabolic biomarkers, amongst others a reduction in serum HDL cholesterol 
concentrations (8). Our in vitro studies described in this thesis were the first showing that antibiotic treatment could directly influence ApoA-I transcription and secretion in human liver and intestinal cells. Moreover, in line with several human and animal studies (9-12), we revealed favorable effects of SCFAs (linking to potential indirect effects of antibiotic treatment) as well as specific amino acids on hepatic ApoA-I transcription. Our findings further provide a mechanistic insight underlying the effects of these compounds on ApoA-I transcription. Sharing these research findings within the scientific community helps to evolve our understanding of the underlying processes and the regulatory factors involved in ApoA-I transcription and to develop strategies targeting ApoA-I transcription via dietary approaches. Therefore, it is important that the data presented in this thesis is available to the (scientific) public through publications in international scientific journals. Also, our preliminary results were presented at national and international conferences that support the development of fundamental knowledge and created the possibility to discuss the findings with several experts from the field. Altogether, this stimulates that the leads we presented here can also be further developed by others. This thesis was funded by a scholarship granted by university of Jeddah, a leading governmental institution of higher education in Saudi Arabia. This implies that the collaboration between the university of Jeddah and Maastricht university contributes to obtain new international scientific perspectives, transfer knowledge between research institutions and to gain new insights into CVD treatment and prevention.

\section{Translation into clinical application}

The studies described in this thesis are in vitro experiments using different cell lines, which means that our observations cannot be directly used as clinical applications. First, our findings need to be confirmed in additional (in vivo) experiments. In other words, this research alone is not enough to adapt the dietary guidelines for fiber or protein intake or to recommend any dietary adjustments for the food industry. If our findings could be confirmed in human interventions, i.e. elevating the uptake of specific SCFAs and/or amino acids effectively increased ApoA-I and HDL functionality, this will be of high relevance for nutritional interventions targeting ApoA-I. However, more studies are needed to identify the most effective dietary fibers (that need to be processed into the optimal SCFAs by our microbiota) or proteins (that need to contain the optimal amino acids composition), thereby stimulating similar effect as reported in our in vitro experiments. Into more detail, after the confirmation of these positive findings in human studies, the intake of SCFAs or amino acids could be used by health care professionals as a dietary recommendation to 
prevent the development of CVD in general, or for example as adjunct therapy for patients treated with antibiotics or by the food industries for the development of novel functional foods.

To conclude, this thesis provides a first insight into the potential role of SCFAs and amino acids on ApoA-I transcription and secretion in human cell line models. Our finding can be used to design future human interventions to investigate the effects of SCFAs and amino acids in clinical applications. Finally, these positive effects might contribute to the development of new nutritional strategies to improve the HDL functionality and subsequently to reduce CVD risk.

\section{References}

1. Mach, F.; Baigent, C.; Catapano, A. L.; Koskinas, K. C., et al. 2019 ESC/EAS Guidelines for the management of dyslipidaemias: lipid modification to reduce cardiovascular risk: The Task Force for the management of dyslipidaemias of the European Society of Cardiology (ESC) and European Atherosclerosis Society (EAS). Eur. Heart J. 2020, 41, 111-188.

2. Rader, D. J.; Hovingh, G. K. HDL and cardiovascular disease. Lancet 2014, 384, 618-625.

3. He, Y.; Kothari, V.; Bornfeldt, K. E. High-Density Lipoprotein Function in Cardiovascular Disease and Diabetes Mellitus. Arterioscler. Thromb. Vasc. Biol. 2018, 38, e10-e16.

4. Rothblat, G. H.; Phillips, M. C. High-density lipoprotein heterogeneity and function in reverse cholesterol transport. Curr. Opin. Lipidol. 2010, 21, 229-238.

5. Rohatgi, A. Reverse Cholesterol Transport and Atherosclerosis. Arterioscler. Thromb. Vasc. Biol. 2019, 39, 2-4.

6. Frank, P. G.; Marcel, Y. L. Apolipoprotein A-I: structure-function relationships. J. Lipid Res. 2000, 41, 853-872.

7. Di Bartolo, B. A.; Scherer, D. J.; Nicholls, S. J. Inducing apolipoprotein A-I synthesis to reduce cardiovascular risk: from ASSERT to SUSTAIN and beyond. Arch. Med. Sci. 2016, 12, 1302-1307.

8. De Smet, E. Plant stanol esters: focus on intestinal lipoprotein metabolism. 2014. (Doctoral dissertation), Maastricht University, Maastricht, Netherlands. Available from BOXPress (ISBN: 978-90-8891-909-1).

9. Blaak, E. E.; Canfora, E. E.; Theis, S.; Frost, G.; Groen, A. K.; Mithieux, G.; Nauta, A.; Scott, K.; Stahl, B.; van Harsselaar, J.; van Tol, R.; Vaughan, E. E.; Verbeke, K. Short chain fatty acids in human gut and metabolic health. Benef. Microbes. 2020, 11, 411-455.

10. Lu, Y.; Fan, C.; Li, P.; Lu, Y.; Chang, X.; Qi, K. Short Chain Fatty Acids Prevent High-fat-dietinduced Obesity in Mice by Regulating G Protein-coupled Receptors and Gut Microbiota. Sci. Rep. 2016, 6, 37589.

11. Jonker, R.; Engelen, M. P. K. J.; Deutz, N. E. P. Role of specific dietary amino acids in clinical conditions. Br. J. Nutr. 2012, 108, S139-S148.

12. Bifari, F.; Ruocco, C.; Decimo, I.; Fumagalli, G.; Valerio, A.; Nisoli, E. Amino acid supplements and metabolic health: a potential interplay between intestinal microbiota and systems control. Genes Nutr. 2017, 12, 27. 

Abbreviations 

ABCA1

AP-1

ApoA-I

BET

Caco-2

cDNA

C/EBP- $\beta$

CETP

CPT1

CVD

DMEM

DMSO

ELISA

ER

FBS

HDL

HDAC

HepG2

IL-1 $\beta$

IL-8

KEAP1

LCAT

LDL

LPS

MEM

mRNA

NF- $\kappa B$

PPARa

PPRE

q-PCR

RCT

RXR

SCFAs

SREBP1

Thap

TG

TLR5

$\mathrm{TNF} \alpha$
ATP-binding cassette transporter A1

Activator protein 1

Apolipoprotein A-I

Bromodomain and Extra-Terminal domain

Human colonocytes

Complementary DNA

CCAAT/enhancer binding protein $\beta$

Cholesteryl ester transfer protein

Carnitine palmitoyl transferase $1 \mathrm{a}$

Cardiovascular diseases

Dulbecco's modified eagle medium

Dimethyl sulfoxide

Enzyme-linked sandwich immunoassay

Endoplasmic reticulum

Fetal bovine serum

High-density lipoprotein

Histone deacetylase

Human hepatocytes

Interleukin 1 beta

Interleukin 8

Kelch like ECH associated protein 1

Lecithin cholesterol acyltransferase

Low-density lipoprotein

Lipopolysaccharide

Minimum essential medium

Messenger RNA

Nuclear factor kappa B

Peroxisome Proliferator Activated Receptor Alpha

PPAR response element

Quantitative polymerase chain reaction

Reverse cholesterol transport

Retinoid X receptor

Short-chain fatty acids

Sterol regulatory element-binding protein 1

Thapsigargin

Triglyceride

Toll-like receptor 5

Tumor necrosis factor- $\alpha$ 

Acknowledgements 

I would like to thank everyone helped me in my $\mathrm{PhD}$ project, whether in the practical part of the research or in the writing part, which resulted in this final thesis. I feel grateful for being a part of the $\mathrm{PHuN}$ research group.

First, I would like to thank my great promotores. Dear Jogchum, there are not enough words to express my gratitude to you for your efforts over the past years in supporting and guiding me to continue the hard work and progress to obtain the best results, also you were spreading optimism and positivity, which was an important reason to achieve the goals required in my research. Dear Ronald, you have been a great supporter of all my studies as your guidance was important to improve the scientific quality of my articles and without your efforts these articles will not be published in excellent international scientific journals. Dear Herman, thank you for helping and teaching me the basic lab skills, this was an important reason for my success in achieving this stage. I remember when I was started my master program in the department, I had started from zero in the laboratories skills, but you were patient and taught me everything that needed to perform my research.

Dear Maurice, I want to specially thank you for your great efforts that you assisting me in all of my steps during this research and I appreciate your tolerance and patience for all the mistakes that I made in the lab during the previous years, but the good thing that at the end these mistakes resulted in several of published articles under the name of our group. Dear Maud, I will not forget your help and your participation in many parts of my research, and the analysis results that you providing me were always positive and encouraging for more experiments.

Dear Kim and Fatma, I was happy to work with both of you on my own research, and the experiments we did together are now an important part of this thesis. I wish you both a good luck in your future researches.

Many thanks to Prof. Dr. E.C.M. Mariman, Prof. Dr. R. Shiri-Sverdlov, Dr. E. Canfora, Prof. Dr. S.A.H. Kersten and Prof. Dr. J. Garssen for your time to evaluate and review my thesis as well as for being present at the official $\mathrm{PhD}$ defense.

My paranymphs from "PHuN group" Kevin and Marco, thank you for your time for planning and organizing the defense details.

To all my former and current colleagues: Sophie, Cara, Charlotte, Lotte, Dorien, Sabine, Eva, Jose, Elske, Lea, Kylie, Merel, Nathalie, Sultan, Fatma, Jordi, Lieve, Ellen, Kevin, Maite, Mathijs, Peter, Tanja, Marco, Gabrielle, Johanna, Sanne, Micah, Willem and Janna thank you for every moment I have here. I am proud and more than honored to be a part of this big family. 
Kenneth Pasmans, Rens, Manuel, Adriyan, Lars, Jacco, Harry, Irene, Kyra and Rodrigo thanks for your kind friendship and supports during my $\mathrm{PhD}$.

I would also like to thank the secretaries, in addition to the colleagues in our department, for all their help. This has been a great life experience, thank you for your kindness, also I will miss the Vlaai meetings.

To my family, my steps would not be this far without the great support and continuous prayers from my parents and all of my family in Saudi Arabia. Thanks for the endless phone and video calls during the past years. Thank you, my wife for your unlimited supports and taking care of our children during our PhD. It was a big challenge, but you succeed and dealt with these difficult times.

Finally, sincere gratitude to the university of Jeddah, which has granted me a scholarship and made this $\mathrm{PhD}$ journey possible. 



\section{Curriculum vitae List of publications}





\section{Curriculum vitae}

Jehad Zuhair Tayyeb was born on December $3^{\text {rd }}, 1985$ in Birmingham, United Kingdom. He was raised in Jeddah, Saudi Arabia and completed his bachelor's (MBBS) degree in medicine and surgery at King Abdulaziz University in 2011. During undergraduate studies he enrolled in several internships in internal medicine, surgery, pediatrics, obstetricsgynecology, emergency medicine, ophthalmology, psychiatry and radiology in King Abdulaziz University hospital. He continued his education in the Netherlands, where he obtained

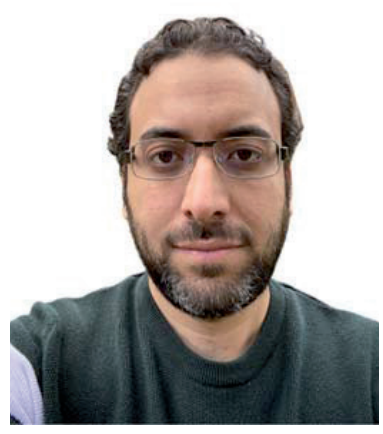
his master's degree in Biomedical Sciences at Maastricht University in 2016 at the Faculty of Health, Medicine and Life sciences. In 2016, Jehad started his PhD at Maastricht University within NUTRIM School of Nutrition and Translational Research in Metabolism under the supervision of Prof. Dr. Jogchum Plat, Prof. Dr. Ronald Mensink and Dr. Herman Popeijus. The research that was performed during this $\mathrm{PhD}$ project is described in this dissertation, entitled "the effects of antibiotics, short-chain fatty acids and amino acids on Apolipoprotein A-I transcription and synthesis in normal and inflamed HepG2 and Caco-2 cells". During his $\mathrm{PhD}$, Jehad has presented his work as oral presentations in national symposiums (The Dutch Nutritional Science Days, NSD from 2016 to 2019, Heeze) and international conferences (European Atherosclerosis Society Congress, EAS 2019, Maastricht). 


\section{List of publications}

Van der Krieken SE, Popeijus HE, Bendik I, Bohlendorf B, Konings MC, Tayyeb JZ, Mensink RP, Plat J. Large-Scale Screening of Natural Products Transactivating Peroxisome Proliferator-Activated Receptor a Identifies 9S-Hydroxy-10E,12Z,15Z-Octadecatrienoic Acid and Cymarin as Potential Compounds Capable of Increasing Apolipoprotein A-I Transcription in Human Liver Cells. Lipids. 2018.

Tayyeb JZ, Popeijus HE, Mensink RP, Konings MC, Mulders KH, Plat J. The effects of short-chain fatty acids on the transcription and secretion of apolipoprotein A-I in human hepatocytes in vitro. J. Cell. Biochem. 2019.

Tayyeb JZ, Popeijus HE, Mensink RP, Konings MC, Mulders KH, Plat J. Amoxicillin Modulates ApoA-I Transcription and Secretion, Predominantly via PPARa Transactivation Inhibition. Int. J. Mol. Sci. 2019.

Tayyeb JZ, Popeijus HE, Mensink RP, Konings MC, Mokhtar FBA, Plat J. Short-Chain Fatty Acids (Except Hexanoic Acid) Lower NF-kB Transactivation, Which Rescues InflammationInduced Decreased Apolipoprotein A-I Transcription in HepG2 Cells. Int. J. Mol. Sci. 2020.

Tayyeb JZ, Popeijus HE, Mensink RP, Plat J. Butyric acid added apically to intestinal Caco2 cells elevates hepatic ApoA-I transcription and rescues the lower ApoA-I expression in the inflamed HepG2 cells co-cultured in the basolateral compartment. Biomolecules. 2021

Tayyeb JZ, Popeijus HE, Mensink RP, Plat J. Differential effects of individual amino acids on PPARa transactivation and the consequent changes in ApoA-I transcription and (pro)ApoA-I secretion. To be submitted. 
